\title{
Are High-redshift Galaxies Hot? Temperature of $z>5$ Galaxies and Implications for Their Dust Properties
}

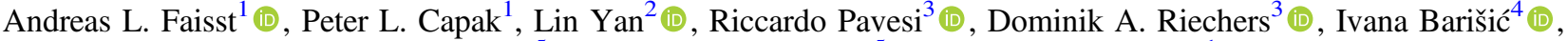 \\ Kevin C. Cooke ${ }^{5}$ (D) Jeyhan S. Kartaltepe ${ }^{5}$, and Daniel C. Masters ${ }^{1}$ (iD \\ ${ }^{1}$ Infrared Processing and Analysis Center, California Institute of Technology, Pasadena, CA 91125, USA; afaisst@ipac.caltech.edu \\ ${ }^{2}$ Cahill Center for Astronomy and Astrophysics, California Institute of Technology, Pasadena, CA 91125, USA \\ ${ }^{3}$ Department of Astronomy, Cornell University, Space Sciences Building, Ithaca, NY 14853, USA \\ ${ }^{4}$ Max-Planck Institut für Astronomie, Königstuhl 17, D-69117, Heidelberg, Germany \\ ${ }^{5}$ School of Physics and Astronomy, Rochester Institute of Technology, 84 Lomb Memorial Drive, Rochester, NY 14623, USA \\ Received 2017 June 1; revised 2017 August 4; accepted 2017 August 22; published 2017 September 15
}

\begin{abstract}
Recent studies have found a significant evolution and scatter in the relationship between the UV spectral slope ( $\left.\beta_{\text {UV }}\right)$ and the infrared excess (IRX; $L_{\mathrm{IR}} / L_{\mathrm{UV}}$ ) at $z>4$, suggesting different dust properties of these galaxies. The total farinfrared (FIR) luminosity is key for this analysis, but it is poorly constrained in normal (main-sequence) star-forming $z>5$ galaxies, where often only one single FIR point is available. To better inform estimates of the FIR luminosity, we construct a sample of local galaxies and three low-redshift analogues of $z>5$ systems. The trends in this sample suggest that normal high-redshift galaxies have a warmer infrared (IR) spectral energy distribution (SED) compared to average $z<4$ galaxies that are used as priors in these studies. The blueshifted peak and mid-IR excess emission could be explained by a combination of a larger fraction of metal-poor interstellar medium being optically thin to ultraviolet (UV) light and a stronger UV radiation field due to high star formation densities. Assuming a maximally warm IR SED suggests a 0.6 dex increase in total FIR luminosities, which removes some tension between the dust attenuation models and observations of the IRX $-\beta$ relation at $z>5$. Despite this, some galaxies still fall below the minimum IRX $-\beta$ relation derived with standard dust cloud models. We propose that radiation pressure in these highly star-forming galaxies causes a spatial offset between dust clouds and young star-forming regions within the lifetime of O/B stars. These offsets change the radiation balance and create viewing-angle effects that can change UV colors at fixed IRX. We provide a modified model that can explain the location of these galaxies on the IRX $-\beta$ diagram.
\end{abstract}

Key words: dust, extinction - galaxies: formation - galaxies: ISM

\section{Introduction}

Modern galaxy surveys have provided large and robust samples of $z>5$ galaxies that span a wide range of properties. These rich data sets are beginning to give us insights into the physical properties of early galaxies only one billion years after the big bang. In particular, the specific star formation rate (sSFR; the rate of mass build-up) is found to increase rapidly by more than a factor of 50 with increasing redshift at $z<2$ (e.g., Daddi et al. 2007; Elbaz et al. 2007; Noeske et al. 2007; Karim et al. 2011; Ilbert et al. 2015) and is expected to keep rising continuously out to $z \sim 6$ (Schaerer et al. 2013; Stark et al. 2013; de Barros et al. 2014; González et al. 2014; Tasca et al. 2015; Faisst et al. 2016a; Jiang et al. 2016; Mármol-Queraltó et al. 2016). Although the average low-redshift star-forming galaxy is represented by a smooth bulge/ disk profile, galaxies in the early universe are irregular with turbulent clumps in the ultraviolet (UV) light representing regions of ongoing vigorous star formation (e.g., Elmegreen et al. 2009; Förster Schreiber et al. 2011; Hemmati et al. 2014; Tacchella et al. 2015; Mason et al. 2016). Furthermore, the amount of dustobscured star formation increases significantly between $0<z<4$, where it can be measured directly and appears to begin declining at $z>4$ based on UV colors, indicating a change in the interstellar medium (ISM) properties of galaxies in the early universe (e.g., Bouwens et al. 2009, 2012; Feldmann 2015). However, the farinfrared (FIR) properties of normal $^{6}$ main-sequence $z>5$ galaxies

\footnotetext{
6 As opposed to submillimeter galaxies (SMGs) and dusty star-forming galaxies (DSFGs) for which rich data sets in the FIR are available out to high redshifts.
}

have been difficult to study even with the Atacama Large Millimeter Array (ALMA), which mostly provides only one single data point at these wavelengths.

The IRX - $\beta$ diagram (Meurer et al. 1995, 1999) is one of the few probes available to date to study the ISM properties of large samples of normal $z>5$ galaxies within reasonable observation times on the facilities that are currently available. This diagram connects the ratio of total FIR to UV luminosity ${ }^{7}$ $\left(L_{\mathrm{FIR}} / L_{\mathrm{UV}} \equiv \mathrm{IRX}\right)$ with the UV continuum spectral slope $(\beta){ }^{8}$ While the former is a proxy of the total dust mass, the latter depends on the column density of dust along the line of sight to the observer that is attenuating the UV light of stars and hence creating a red UV color. The relation is therefore sensitive to a range of ISM properties including dust geometries, dust-to-gas ratios, dust grain properties, and the spatial distribution of dust. Furthermore, the scatter and trends in this diagram are strongly related to evolutionary trends in the ISM. Furthermore, the UV slope $\beta$ also depends on other galaxy properties such as star formation history (SFH), age of the stellar population, and metallicity. However, in comparison to dust, these effects are shown to contribute little to $\beta$ in the case of young galaxies in the early universe (Bouwens et al. 2012; Scoville et al. 2015).

Studies of low-redshift galaxies have shown that galaxies of different types populate different regions of the infrared excess

\footnotetext{
The UV luminosity $\left(L_{\mathrm{UV}}\right)$ is defined as the monochromatic luminosity measured at rest-frame $1600 \mathrm{~A}$. The total FIR luminosity $\left(L_{\mathrm{FIR}}\right.$; sometimes also called "TIR") is integrated at 3-1100 $\mu \mathrm{m}$.

8 The UV continuum slope (defined as $f_{\lambda} \propto \lambda^{\beta}$ ) is derived for $1600 \AA$ $<\lambda<2600 \AA$ (e.g., Meurer et al. 1999).
} 
(IRX) - $\beta$ diagram. Specifically, young metal-poor galaxies similar to the Small Magellanic cloud (SMC) show a flatter relation between IRX and $\beta$ compared to local starburst galaxies, which occupy regions of higher IRX at similar $\beta$ (e.g., Buat et al. 2005; Siana et al. 2009; Buat et al. 2010; Howell et al. 2010; Reddy et al. 2010; Takeuchi et al. 2010; Overzier et al. 2011; Boquien et al. 2012; Reddy et al. 2012; Battisti et al. 2017). The differences between these populations of galaxies on the IRX $-\beta$ diagram can be attributed to differences in the shape of the internal dust attenuation curves (e.g., Gordon et al. 2000; Burgarella et al. 2005; Buat et al. 2012; Battisti et al. 2017), different star formation histories (e.g., Kong et al. 2004; Boquien et al. 2009; Muñoz-Mateos et al. 2009), and/or different dust geometries (Seibert et al. 2005; Cortese et al. 2006; Boquien et al. 2009; Muñoz-Mateos et al. 2009). Studies at $z<3$ suggest IRX $-\beta$ relations similar to local starbursts with no significant evolution (e.g., Reddy et al. 2006, 2012; Heinis et al. 2013; To et al. 2014; Bourne et al. 2017; Fudamoto et al. 2017); however, stacking analyses at $z>3$ suggest significant deviations from this relation for luminous and young Lyman Break galaxies (Lee et al. 2012; Coppin et al. 2015; Fudamoto et al. 2017). Although the Hubble Space Telescope (HST) and ground-based facilities provide us with accurate measurements of the rest-frame UV properties of high-redshift galaxies, the high sensitivity of millimeter-wave interferometers such as ALMA, the Plateau de Bure Interferometer, and its successor the Northern Extended Millimeter Array enable us to push the measurement of the FIR properties of individual galaxies to higher and higher redshifts (Walter et al. 2012; Kanekar et al. 2013; Ouchi et al. 2013; González-López et al. 2014; Ota et al. 2014; Riechers et al. 2014; Capak et al. 2015; Maiolino et al. 2015; Schaerer et al. 2015; Watson et al. 2015; Willott et al. 2015; Aravena et al. 2016; Bouwens et al. 2016; Carilli et al. 2016; Dunlop 2016; Knudsen et al. 2016; Miller et al. 2016; Pavesi et al. 2016; Knudsen et al. 2017).

Capak et al. (2015; hereafter C15) provided the first study of the IRX- $\beta$ diagram at $z>5$ with a diverse sample of $5.1<z<5.7$ galaxies observed in ALMA band 7 at restframe $\sim 150 \mu \mathrm{m}$. Together with deep HST near-IR imaging providing accurate UV colors for this sample (Barišić et al. 2017), these studies suggest a significant evolution of the IRX $-\beta$ relation and its scatter at $z>5$ (see also Fudamoto et al. 2017). Although some galaxies are found to be consistent with the relation of local starbursts, more than half of the galaxies show a substantial deficit in IRX at a range of UV colors compared to the samples at $z<3$. Such galaxies are curious because their location on the IRX $-\beta$ diagram is difficult to explain with current models for dust attenuation (Charlot \& Fall 2000) even for very low dust opacities and steep internal dust attenuation curves such as those observed in the metalpoor SMC.

Because of the poor constraints in the FIR, a common (partial) solution to this discrepancy is to assume increasing dust temperatures toward high redshifts (Capak et al. 2015; Bouwens et al. 2016; Fudamoto et al. 2017; Narayanan et al. 2017). However, a significantly altered geometric distribution of stars and dust in these galaxies could also explain their low IRX values.

In this paper, we try to understand the causes of such low IRX values in this high-redshift sample by exploring the infrared (IR) spectral energy distribution (SED) and the distribution of dust and stars.

We begin by investigating the FIR luminosities of these galaxies, which are derived from only one continuum data point at $\sim 150 \mu \mathrm{m}$ and are therefore very poorly constrained. Importantly, the shape of the IR SED is assumed from models that are fit at $z<4$. In particular, the luminosity-weighted temperature $T$ (referred to as "temperature" in this paper and not to be confused with the single blackbody temperature $T_{\text {peak }}$; see Casey 2012) which accounts for the mid-IR excess emission is crucial for defining the shape of the IR SED and therefore the FIR luminosity. Hence, it has a significant impact on the IRX $-\beta$ relation. Several studies suggest and expect a higher temperature in highly star-forming high-redshift galaxies due to a stronger UV radiation field in low-metallicity environments (Sklias et al. 2014; Béthermin et al. 2015; Strandet et al. 2016; Schreiber et al. 2017) and as a function of various galaxy properties (Chapman et al. 2003; Magdis et al. 2012; Magnelli et al. 2014). Such an evolving temperature could bring these galaxies in agreement with local IRX $-\beta$ relations (Capak et al. 2015; Bouwens et al. 2016; Fudamoto et al. 2017; Narayanan et al. 2017). A direct measurement of this temperature is, however, very difficult to obtain for main-sequence star-forming galaxies at $z>5$ (see Appendix). Hence, our best chance to make progress is to investigate statistically the correlations between the temperature and other physical properties in large samples of lowredshift galaxies together with the study of low-redshift analogues of such $z>5$ galaxies. We stress that this is by no means a bulletproof approach, but it allows us to gain a picture of galaxies in the early universe and will lead and define follow-up explorations of these galaxies with future facilities that can refine the conclusions of this work.

Furthermore, the geometry of the dust distribution in highredshift galaxies could be substantially different due to their turbulent nature-something that is mostly not considered in recent studies. As we will show in this paper, this can lead naturally to a low IRX value and a large range of UV colors and thus can explain extreme cases in the galaxy population at high redshifts.

This work is organized as follows. In Section 2, we present FIR measurements and their correlation with other physical properties for local galaxies from the literature along with three $z \sim 0.3$ Ly $\alpha$-emitting galaxies, which appear to be good analogues of high-redshift $(z>5)$ galaxies. We then investigate the impact of different galaxy properties on the IRX $-\beta$ relations from a model and observational point of view, which will allow us to explain empirically a possible evolution of the IRX $-\beta$ relation to high redshifts (Section 3). In Section 4, we revisit the $\mathrm{C} 15$ galaxies, derive FIR luminosities using the priors on the IR SED shape from the low-redshift samples, and present an updated IRX $-\beta$ diagram at $z \sim 5.5$. Finally, we propose a simple analytical model for the dust distribution in high-redshift galaxies, which can describe the observed deficit in IRX by taking into account a non-uniform distribution of dust and stars (Section 4.5).

Throughout this work, we assume a flat cosmology with $\Omega_{\Lambda, 0}=0.7, \Omega_{m, 0}=0.3$, and $h=0.7$. Stellar masses and SFRs are scaled to a Chabrier (2003) initial mass function (IMF) and magnitudes are quoted in AB (Oke 1974). Metallicities are quoted in the Pettini \& Pagel (2004) calibration unless specified differently. 


\section{FIR Properties of Local and Low-redshift Galaxies}

One way to understand the high-redshift universe is to assemble a sample of low-redshift objects that are "analogues" to the high-redshift population. These analogues typically can provide much more detailed data and can be used to construct priors on the physical properties of high-redshift galaxies. However, a key shortcoming of this technique is that the lowredshift sample is often an analogue in only a few parameters, and so extrapolations must be made. As such, it is essential to carefully study the low-redshift sample and determine how well it matches the higher redshift one.

Here we start with several samples of local galaxies that have a wealth of FIR data from the Herschel observatory and other sources. We explore correlations between their FIR properties and other physical parameters, which are measurable in our $z>5$ sample and distinguish them the most from galaxies at lower redshifts. We then study three $z \sim 0.3$ Ly $\alpha$ emitters that are analogues to the $z>5$ sample in rest-frame UV properties and explore their FIR properties in comparison to the Herschel sample. To ensure consistency, we re-analyze the low-redshift samples using the same techniques we apply at higher redshift.

\subsection{The Sample of Herschel Observed Local Galaxies}

In the following, we consider local galaxies from the KINGFISH sample (Kennicutt et al. 2011), the GOALS sample (Sanders et al. 2003; Armus et al. 2009), and the Dwarf Galaxy Survey (DGS; Madden et al. 2013). Combining these samples allows us to cover a wide range in galaxy properties. Here, we briefly summarize the main properties of these samples. We note that we have removed all galaxies with a known active galactic nucleus (AGN) from these three samples; however, we cannot rule out the presence of heavily obscured AGNs (see discussion in Section 2.4).

1. The KINGFISH sample consists of 61 nearby galaxies within $30 \mathrm{Mpc}$ with $9<\log \left(M / M_{\odot}\right)<10$ but also contains a handful of less massive dwarf galaxies at $10^{7} M_{\odot}$ (Skibba et al. 2011). Their sSFRs are predominantly $0.1-1.0 \mathrm{Gyr}^{-1}$, and the metallicities are in the range of $12+\log (\mathrm{O} / \mathrm{H}) \sim$ 8.5-8.8 (Kennicutt et al. 2011). All KINGFISH galaxies are observed with Spitzer/IRAC (3.6, 4.5, 5.8, and $8.0 \mu \mathrm{m})$ and Spitzer/MIPS (24 and $70 \mu \mathrm{m})$ as well as Herschel/PACS (70, 110, and $160 \mu \mathrm{m})$ and Herschel/SPIRE (250, 350, and $500 \mu \mathrm{m}$; Temi et al. 2009; Dale et al. 2012; Bendo et al. 2012).

2. The GOALS sample consists of $\sim 200$ of the most luminous infrared-selected galaxies including merging systems in the nearby universe (LIRGs) out to $400 \mathrm{Mpc}$ (Kennicutt et al. 2011). In particular, about 20 of these are ultraluminous infrared galaxies (ULIRGs). The GOALS galaxies have stellar masses mostly between $10<\log \left(M / M_{\odot}\right)<11.5$ and are therefore the most massive galaxies of these three samples (U et al. 2012). Furthermore, the galaxies span a wide range in sSFR from $0.1 \mathrm{Gyr}^{-1}$ up to $10 \mathrm{Gyr}^{-1}$ for the lowest masses $\left(\log \left(M / M_{\odot}\right) \sim 9.5\right)$. There are only a handful of metallicity measurements for these galaxies, and they are in general around $12+\log (\mathrm{O} / \mathrm{H})=8.5$ (Rupke et al. 2008; Rich et al. 2012). Most of the GOALS galaxies are observed by Spitzer/IRAC and Spitzer/MIPS as well as Herschel/PACS and Herschel/SPIRE (Sanders et al.
2003; U et al. 2012; Díaz-Santos et al. 2014; Chu et al. 2017).

3. The DGS sample consists of 50 low-mass $(7<$ $\left.\log \left(M / M_{\odot}\right)<10\right)$ dwarf galaxies in the nearby universe out to $200 \mathrm{Mpc}$ (Madden et al. 2013). The galaxies populate mostly low metallicities $(12+\log (\mathrm{O} / \mathrm{H}) \sim$ 7.8-8.7; Rémy-Ruyer et al. 2013) and are therefore similar to high- $z$ galaxies and our low-redshift analogues, with the exception that the DGS galaxies have a lower sSFR (0.1-1.0 $\mathrm{Gyr}^{-1}$ ), similar to the KINGFISH sample (Madden et al. 2013; Cormier et al. 2015). All of the DGS galaxies are observed with Spitzer/IRAC and Spitzer/ MIPS as well as Herschel/PACS and Herschel/SPIRE (Bendo et al. 2012; Rémy-Ruyer et al. 2013).

\subsubsection{Parameterization of the IR SED and Fitting of Temperature}

We measure the FIR properties of the local galaxies using the parameterization introduced by Casey (2012) and their public IR photometry from 24 to $500 \mu \mathrm{m}$. We will use this parameterization throughout this work to ensure consistent measurements for all galaxy samples discussed in the following.

The Casey (2012) parameterization combines a single graybody (accounting for the reprocessed, cold to warm emission) with a mid-IR power law (approximating the warm to hot dust components from AGN heating, hot star-forming regions, and optically thin dust). As shown in their study, this method results in a better fit to the rest-frame mid-IR part of the spectrum by marginalizing over multiple warm to hot dust components of a galaxy (see their Figure 1 for an illustration) compared to a single graybody. Mathematically, the parametrization depends on the luminosity-weighted temperature $(T)$, the slope of the mid-IR power-law component $(\alpha)$, the emissivity $\left(\beta_{\mathrm{IR}}\right)$, and a normalization.

Note that $T$ represents the average temperature marginalized over all warm temperature components combined in the IR SED and is therefore a luminosity-weighted temperature (in the following referred to as just "temperature" for convenience). This temperature should not be confused with the peak temperature ${ }^{9} T_{\text {peak }}$ that is quoted in some other studies and is only equivalent to $T$ only in the case of a single blackbodyshaped IR SED (in general, it is always $T>T_{\text {peak }}$; see the detailed discussion in Casey 2012). In this work, we study the luminosity-weighted temperature $T$; however, for convenience, we quote equivalent $T_{\text {peak }}$ temperatures whenever possible using the relation shown in Casey (2012). Importantly, $T$ defines the shape of the IR SED by taking into account the midIR emission. It is not only affected by the temperature of the optically thick dust, but also by the opacity of the dust in the ISM of a galaxy as well as dust grain properties, which can significantly change the mid-IR slope $\alpha$ as shown by Scoville $\&$ Kwan (1976) and further discussed in Section 2.4. Therefore, changes in the temperature $T$ are not only limited to changes in the dust temperature, but also include changes in the opacity of the dust or its geometry. Hence, we refer to $T$ for all of these effects describing the shape of the IR SED rather than solely to the dust temperature.

For the fitting of the IR SED using the above parameters, we fix the wavelength at which the optical depth is unity to $\lambda_{0}=200 \mu \mathrm{m}$ (Draine 2006; Conley et al. 2011; Casey 2012;

\footnotetext{
9 For example, computed using Wien's displacement law $T_{\text {peak }}=b / \lambda_{\text {peak }}$ with $b=2898 \mu \mathrm{m} \mathrm{K}$.
} 
Riechers et al. 2013). This is somewhat arbitrary as $\lambda_{0}$ is a priori unknown and is likely to change for different samples of galaxies, although Riechers et al. (2013) find a consistent value close to $200 \mu \mathrm{m}$ for $z>4$ SMGs. Using $\lambda_{0}=100 \mu \mathrm{m}$ would result in $\sim 5 \mathrm{~K}$ cooler temperatures, but does not change our results for the total FIR luminosity, so the choice is irrelevant as long as it is treated consistently. For all fits, we assume a freely varying $\beta_{\mathrm{IR}}$. However, because $\beta_{\mathrm{IR}}$ and $T$ can be degenerate, we also fit the photometry with a fixed $\beta_{\text {IR }}$ of 1.6 and 2.0 but we do not find any significant differences in the following results and conclusions.

\subsection{Low-redshift Analogues of High-z Galaxies}

In addition to the Herschel samples, we consider three galaxies at low redshift $(z \sim 0.3)$ that were selected as UV analogues to $z>5$ systems with archival FIR and ALMA observations. Such galaxies are extremes among low-redshift galaxies but show very similar properties to high-redshift galaxies in terms of sSFRs, optical emission lines, metallicity, and morphology. Low-redshift analogues, such as "Green Peas" (Cardamone et al. 2009) or ultrastrong emission line galaxies (Hu et al. 2009), are therefore used by many studies to understand in detail the population of high-redshift galaxies, for which much less observational data exist (Stanway et al. 2014; Bian et al. 2017; Erb et al. 2016; Faisst 2016; Faisst et al. 2016a; Greis et al. 2016; Masters et al. 2016). A very common property of these galaxies is their high $\mathrm{H} \alpha$ equivalent width (equivalent to sSFR). In fact, as demonstrated by Faisst (2016), the $\mathrm{H} \alpha$ equivalent width allows a very clean selection of lowredshift analogues with very similar properties to high-redshift galaxies.

The three analogues of high-redshift galaxies (hereafter named GALEX 0959+0151, GALEX 1000+0157, and GALEX 1000+0201) were originally selected from the Galaxy Evolution Explorer (GALEX; Martin et al. 2005) Ly $\alpha$-emitter sample described in Cowie et al. (2011; see also Cowie et al. 2010).

This Ly $\alpha$-emitter sample contains galaxies that are spectroscopically preselected to have rest-frame $\mathrm{EW}(\mathrm{Ly} \alpha)>15 \AA$ in the GALEX far-UV or near-UV grism. Galaxies with a clear AGN signature were removed based on a cut in the Ly $\alpha$ FWHM and another cut in the high-ionization optical emission lines (see also Figure 1). All three galaxies are in the main Cosmic Evolution Survey (COSMOS; Scoville et al. $2007)^{10}$ area and thus are imaged by ground- and space-based facilities in more than 30 photometric bands ranging from the UV to radio (Laigle et al. 2016). These are the only galaxies in COSMOS that are analogues of $z>5$ systems with a wealth of ancillary data in the UV, optical, and FIR. Rest-frame optical spectroscopy is available from the zCOSMOS-bright spectroscopic survey (Lilly et al. 2007) ${ }^{11}$ and two of the analogues (GALEX 0959+0151 and GALEX 1000+0157) are observed in the UV with the Cosmic Origin Spectrograph (COS; Green et al. 2012) on HST (C. Scarlata et al. 2017, in preparation).

The three galaxies appear to be good analogues of highredshift systems. Specifically, they show optical emission line properties and Ly $\alpha$ properties similar to galaxies at $z \sim 5$. The $\mathrm{H} \alpha$ equivalent widths $(\mathrm{EW}(\mathrm{H} \alpha)=200-400 \AA)$ are comparable

\footnotetext{
${ }^{10}$ http://cosmos.astro.caltech.edu

11 http://archive.eso.org/cms/eso-data/data-packages/zcosmos-data-releasedr1.html
}



Figure 1. BPT diagram (Baldwin et al. 1981) with our three $z \sim 0.3$ analogues (GALEX 0959+0151, red point; GALEX 1000+0157, orange square; GALEX $1000+0201$, blue diamond). Other samples of analogues are also shown ("Green Peas," green circles; Cardamone et al. 2009), $z \sim 0.8$ ultrastrong emission line galaxies (orange circle; $\mathrm{Hu}$ et al. 2009), and $z \sim 0.2$ Ly $\alpha$ emitters (orange squares; Cowie et al. 2011). The black contours show local galaxies in SDSS and the cyan diamonds show $z \sim 2.3$ galaxies (including best fit) from Steidel et al. (2014). Our analogues show increased $[\mathrm{O}$ III] $/ \mathrm{H} \beta$ and decreased $[\mathrm{N} \mathrm{II}] / \mathrm{H} \alpha$ ratios as expected for galaxies in the early universe. Furthermore, the location of the analogues does not suggest the presence of an AGN.

to those measured in $z \sim 5-6$ systems using Spitzer colors (Shim et al. 2011; Faisst et al. 2016a; Rasappu et al. 2016), and their specific SFRs based on combined UV and FIR luminosities are between 1.6 and $8.8 \mathrm{Gyr}^{-1}$. This is $2-15$ times higher than average galaxies on the star-forming main sequence at $z \sim 0.3$ at stellar masses $\log \left(M / M_{\odot}\right) \sim 9.0-10.0$ (e.g., Speagle et al. 2014; Lee et al. 2015; Schreiber et al. 2015), but similar to those of $z \sim 5-6$ galaxies (e.g., Steinhardt et al. 2014; Capak et al. 2015; Faisst et al. 2016a). Furthermore, the $[\mathrm{O} \mathrm{III}] / \mathrm{H} \beta$ ratios are high (4.5-6.0) and $[\mathrm{N} \mathrm{II}] / \mathrm{H} \alpha$ ratios are low (0.025-0.060) compared to $z \sim 0.3$ galaxies at similar stellar masses, but again comparable to $z \sim 5-6$ systems (see Figure 1 and Kewley et al. 2013; Faisst 2016; Faisst et al. 2016a; Masters et al. 2016). Finally, these line ratios suggest gas-phase metallicities of $12+\log (\mathrm{O} / \mathrm{H})<8.3$ in any calibration, which is 0.3 dex below the average metallicity of $z \sim 0.3$ galaxies at similar stellar mass but comparable to galaxies at $z>3.5$ (see Ando et al. 2007; Kewley \& Ellison 2008; Maiolino et al. 2008; Faisst et al. 2016b).

The detailed spectroscopic properties of the three analogues are listed in Table 1.

\subsubsection{HST Morphology}

Figure 2 shows the optical images from HST's Advanced Camera for Survey (ACS) in F814W (I-band) at $200 \mathrm{pc}$ resolution. The bulk of the optical light is emitted from a compact nucleus of less than $0.8 \mathrm{kpc}$ in diameter but diffuse components extend out to $6 \mathrm{kpc}$ in all of the galaxies. Several clumps of UV light in the diffuse component with size the same as or smaller than the nucleus suggest regions of vigorous star formation. 
Table 1

UV and Optical Spectroscopic Properties of the Three Analogue Galaxies

\begin{tabular}{|c|c|c|c|c|c|c|c|c|c|c|}
\hline Name & $\begin{array}{c}\text { R.A. } \\
\text { (J2000.0) }\end{array}$ & $\begin{array}{c}\text { Decl. } \\
\text { (J2000.0) }\end{array}$ & $\begin{array}{c}z \\
\text { (optical) }\end{array}$ & {$[\mathrm{O} \mathrm{III}] / \mathrm{H} \alpha$} & {$[\mathrm{O} \mathrm{III}] / \mathrm{H} \beta$} & {$[\mathrm{N} \mathrm{II}] / \mathrm{H} \alpha$} & $\begin{array}{c}\mathrm{EW}(\mathrm{Ly} \alpha) \\
(\AA)\end{array}$ & $\begin{array}{c}\mathrm{EW}(\mathrm{H} \alpha) \\
(\AA)\end{array}$ & $12+\log (\mathrm{O} / \mathrm{H})^{\mathrm{a}}$ & $\begin{array}{c}\mathrm{SFR}_{\mathrm{H} \alpha} \\
\left(M_{\odot} \mathrm{yr}^{-1}\right)\end{array}$ \\
\hline GALEX 0959+0151 & 09:59:40.34 & $01: 51: 21.30$ & 0.2506 & $2.11 \pm 0.03$ & $6.04 \pm 0.03$ & $0.051 \pm 0.001$ & $21 \pm 3$ & $333 \pm 4$ & $8.16 \pm 0.01$ & 45 \\
\hline GALEX $1000+0157$ & 10:00:27.85 & 01:57:03.60 & 0.2647 & $1.96 \pm 0.04$ & $5.61 \pm 0.04$ & $0.024 \pm 0.002$ & $43 \pm 2$ & $423 \pm 2$ & $7.98 \pm 0.02$ & 17 \\
\hline GALEX $1000+0201$ & 10:00:35.76 & 02:01:13.50 & 0.2653 & $1.65 \pm 0.03$ & $4.71 \pm 0.03$ & $0.060 \pm 0.001$ & $19 \pm 2$ & $195 \pm 2$ & $8.20 \pm 0.01$ & 11 \\
\hline
\end{tabular}

une.

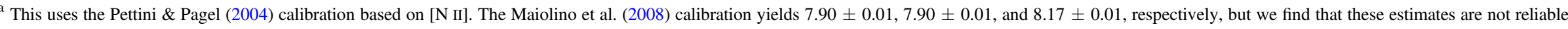
because they are based on the location where the upper and lower branch separate. 


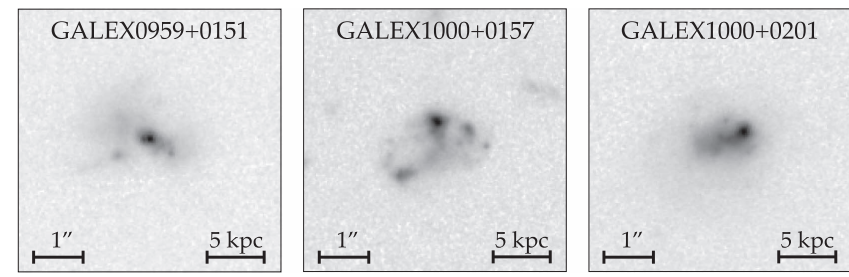

Figure 2. $H S T$ ACS $/ \mathrm{F} 814 \mathrm{~W}$ (rest-frame optical) portraits of the three $z \sim 0.3$ analogues drizzled to a resolution of $0{ }^{\prime \prime} 03 \mathrm{pixel}^{-1}(\sim 200 \mathrm{pc})$. The three galaxies show a disturbed and clumpy structure with indications of recent or ongoing star formation similar to what is expected and observed in highredshift galaxies. The compact nuclei are of the order of $0.8 \mathrm{kpc}$ in diameter but the diffuse components extend out to $\sim 6 \mathrm{kpc}$.

\subsubsection{UV to Mid-IR Data}

The UV to mid-IR photometry of the galaxies is extracted from the COSMOS2015 catalog (Laigle et al. 2016). ${ }^{12}$ Next to deep imaging in the UV and optical, the catalog includes deep Spitzer/IRAC mid-IR data at 3.6 and $4.5 \mu \mathrm{m}$ from the Spitzer Large Area Survey with Hyper-Suprime-Cam (SPLASH; Steinhardt et al. 2014) ${ }^{13}$ as well as Spitzer/MIPS $24 \mu \mathrm{m}$ (all three galaxies are detected). The photometry has been extracted using the positional priors from the optical bands and reliable deblending algorithms (Laigle et al. 2016). The UV to optical SEDs are shown in the top and middle panels of Figure 3. Each of the galaxies has been observed with Spitzer/MIPS at $70 \mu \mathrm{m}$ as well as with Herschel/PACS $(110$ and $170 \mu \mathrm{m})$ and Herschel/SPIRE (250, 350, and $500 \mu \mathrm{m})$. Due to the large point-spread functions and confusion, we choose to measure the IR photometry on a galaxy-by-galaxy basis. In brief, we perform aperture photometry with an appropriate correction to total magnitudes. The uncertainties are based on random parts of the background. For galaxies with close neighbors, we apply either Spitzer/IRAC or Spitzer/MIPS positions as priors and use GALFIT (version 3.0.5; Peng et al. 2011) to carefully subtract the flux of the neighboring galaxies before performing aperture photometry on the main object.

The detailed IR photometry of the three analogues is listed in Table 2.

\subsubsection{Physical Properties from Optical SED Fitting}

We fit the optical SED of our low-redshift analogues to obtain their stellar masses and rest-frame $1600 \AA$ monochromatic luminosities $\left(L_{\mathrm{UV}}\right)$. We use templates based on Bruzual \& Charlot (2003) and include constant, exponentially declining, and increasing SFHs with variable metallicities from $1 / 20$ of solar to solar. The stellar population ages range from $100 \mathrm{Myr}$ to a few Gyr and dust is parametrized by extinction laws based on local starbursts (Calzetti et al. 2000) and the SMC (Prevot et al. 1984; Pettini et al. 1998). However, we found that the different extinction laws do not significantly change the measured physical parameters. The redshift is fixed to the spectroscopic redshift during the fitting. We add strong optical emission lines coupled to $\mathrm{H} \alpha$ with variable ratios [O III] $/ \mathrm{H} \alpha, \mathrm{H} \alpha / \mathrm{H} \beta,{ }^{14}$ and $[\mathrm{O}$ III $] /[\mathrm{O}$ II $]$. Weak

\footnotetext{
12 ftp://ftp.iap.fr/pub/from_users/hjmcc/COSMOS2015/

$13 \mathrm{http}: / /$ splash.caltech.edu

${ }^{14}$ We compute $\mathrm{H} \beta$ via case $\mathrm{B}$ recombination and the fitted $E(B-V)$ value assuming a stellar-to-gas dust ratio of unity as suggested by recent studies (Reddy et al. 2015; Shivaei et al. 2015; De Barros et al. 2016).
}

emission lines (such as $\mathrm{N}, \mathrm{S}$, or $\mathrm{He}$ ) are added with a constant ratio with respect to $\mathrm{H} \beta$ for subsolar metallicity (Anders \& Fritze-v. Alvensleben 2003). The GALEX far-UV photometry is corrected for the Ly $\alpha$ line emission derived from the spectra prior to fitting as we do not include the Ly $\alpha$ line in our SED models. The fit is performed using a LevenbergMarquardt algorithm, as part of the $\mathrm{R} / \mathrm{minpack} . \mathrm{Im}$ package $^{15}$ and errors are obtained from a Monte Carlo sampling taking into account the error in the photometry and models. The UV luminosity $\left(L_{\mathrm{UV}}\right)$ is measured at rest-frame $1600 \AA$, and the UV continuum slope is fitted in the window between 1600 and $2600 \AA$. We perform two fits for accessing the uncertainties in the fitted parameters. First, we fix $\mathrm{EW}(\mathrm{H} \alpha),[\mathrm{O} \mathrm{III}] / \mathrm{H} \alpha$, and $[\mathrm{O} \mathrm{III}] / \mathrm{H} \beta$ to the spectroscopic value, and in a second run, we leave these as free parameters. The fits are shown in Figure 3 on the top and middle panels, respectively, and we find an agreement on the order of $25 \%$ or better between the photometrically and spectroscopically measured $\mathrm{H} \alpha \mathrm{EW}$ and line ratios. We find stellar masses of $\log \left(M / M_{\odot}\right)=9-9.5$, moderate to low dust attenuation $(E(B-V)<0.3)$, and UV luminosities on the order of $\log \left(L_{\mathrm{UV}}\right) \sim 10.2$. The photometrically derived [O III]/ [O II] ratios (ranging from 1.5 to 3.5 ) are factors of 3-6 higher compared to $z \sim 0.3$ galaxies on the main sequence but fit well the expected [O III]/[O II] ratios for $z \sim 5$ galaxies (e.g., Faisst 2016), which is an additional verification of their validity as analogues of high-redshift galaxies. We compare the UV slope $\beta$ measured from SED fitting with the direct measurement from the observed GALEX and CFHT $/ \mathrm{u}^{*}$ fluxes and find a good agreement within the uncertainties. Since we want to study the location of these galaxies on the IRX $-\beta$ diagram, we want the measurement of $\beta$ to be independent from any assumed dust attenuation law. This is not the case for the $\beta$ measurements from SED fitting. We therefore prefer to use the $\beta$ measurement obtained from the observed fluxes.

The physical properties obtained from the optical SED fits are listed in Table 3.

\subsubsection{ALMA Measurements}

The three analogues of high-redshift galaxies have been observed with ALMA in Band 3 (ID: 2012.1.00919.S, PI: Y. Kakazu) centered at an observed frequency of $92 \mathrm{GHz}$, which corresponds to the rest-frame wavelength of $\mathrm{CO}(1-0)$ emission $(2.6 \mathrm{~mm})$. The integration times vary between 1255 and $9852 \mathrm{~s}$ and the number of antennae between 24 and 36 (see Table 2). The line observations are performed in "frequency division mode" at a bandwidth of $0.9375 \mathrm{GHz}$ in one spectral window, and the continuum observations use the "time division mode" with a bandwidth of $2.0 \mathrm{GHz}$ in each of the three line-free spectral windows. The synthesized beam sizes are on average 3 ". $5 \times 2$ " 5 . The data are reduced using the standard CASA ALMA calibration pipeline. All three galaxies are undetected in the continuum around rest-frame $2.6 \mathrm{~mm}$ at $\lesssim 50 \mu \mathrm{Jy}$ at $3 \sigma$, but we note a tentative $\mathrm{CO}(1-0)$ detection at the redshift of GALEX 0959+0151 at a $1 \sigma-2 \sigma$ level with a peak emission spatially offset by 1 !" 8 or $7 \mathrm{kpc}$.

\footnotetext{
${ }^{15}$ https://cran.r-project.org/web/packages/minpack.lm/index.html
} 

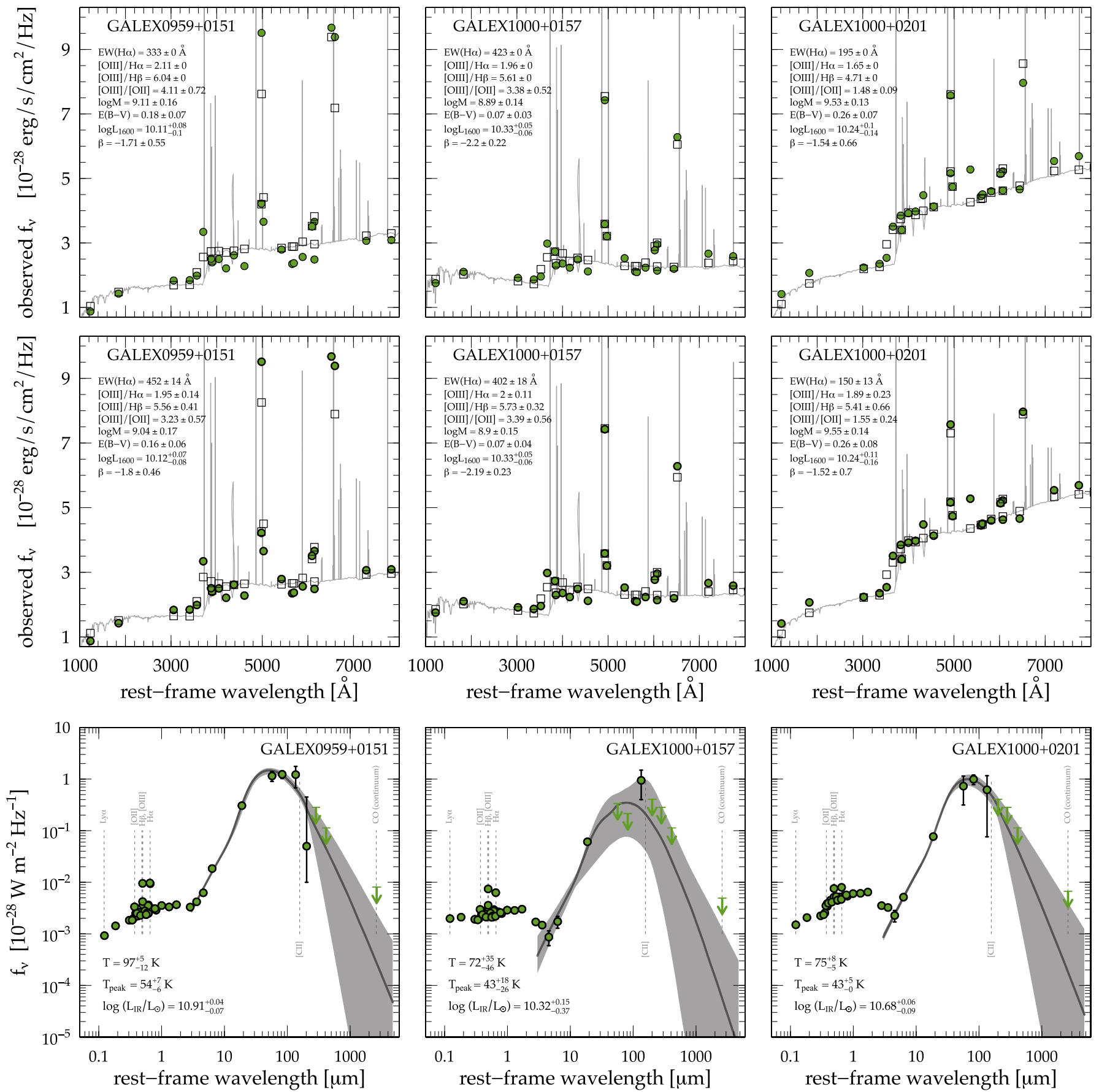

Figure 3. Compilation of UV to optical and IR SEDs of the three $z \sim 0.3$ analogues (green symbols). Top: optical SED fit with [O III]/H $\alpha$ and [O III]/H $\beta$ line ratios as well as $\mathrm{EW}(\mathrm{H} \alpha)$ fixed to the spectroscopically measured values. The open squares show the photometry measured on the best-fit SED. Middle: same as the top panel but with variable line ratios and $\mathrm{EW}(\mathrm{H} \alpha)$. Bottom: IR SED fit from 3 to $3000 \mu \mathrm{m}$ to the photometry given in Table 2 . The green arrows marks upper limits. Optical and FIR emission lines are indicated by vertical lines. The uncertainty of the fit is indicated in gray. The best-fit parameters for the UV to optical and IR fits are given in Tables 3 and 4, respectively.

\subsubsection{IR SEDs and Temperature of the Low-z Analogues}

We estimate the FIR properties of the analogues in the same way as for the Herschel sample (see Section 2.1.1). The bottom panels of Figure 3 show the best-fit IR SEDs to the three analogues (bestfit parameters are listed in Table 4). We find temperatures ranging from 70 to $100 \mathrm{~K}$ with uncertainties of $\sim 10 \mathrm{~K}$ (corresponding to $40-60 \mathrm{~K}$ with uncertainties of $\sim 5 \mathrm{~K}$ in $T_{\text {peak }}$ ). The temperature for GALEX $1000+0157$ is only poorly constrained because only upper limits for the FIR photometry are available.

\subsection{Correlations of FIR Properties with Metallicity and SSFR in Low-redshift Galaxies}

Metallicity and sSFR are the two most prominent ways highredshift galaxies differ from lower-redshift ones. We therefore begin by investigating the dependency of the shape of the IR SED (parameterized by $T$ ) on these physical quantities.

Figure 4 shows the $L_{\mathrm{FIR}}$ versus $T$ and $L_{\mathrm{FIR}}$ versus $T_{\text {peak }}$ relations for our local samples with open symbols color-coded by the metallicities of the galaxies. The different symbols 
Table 2

Photometric IR Properties of the Three Low-redshift Analogues

\begin{tabular}{|c|c|c|c|c|c|c|c|c|}
\hline \multirow{2}{*}{ Name } & \multirow{2}{*}{$\begin{array}{c}\text { Spitzer/MIPS } \\
70 \mu \mathrm{m} \\
(\mathrm{mJy})\end{array}$} & \multicolumn{2}{|c|}{ Herschel/PACS } & \multicolumn{3}{|c|}{ Herschel/SPIRE } & \multicolumn{2}{|c|}{$\mathrm{ALMA}^{\mathrm{a}}$} \\
\hline & & $\begin{array}{c}110 \mu \mathrm{m} \\
(\mathrm{mJy})\end{array}$ & $\begin{array}{c}160 \mu \mathrm{m} \\
(\mathrm{mJy})\end{array}$ & $\begin{array}{l}250 \mu \mathrm{m} \\
(\mathrm{mJy})\end{array}$ & $\begin{array}{c}350 \mu \mathrm{m} \\
(\mathrm{mJy})\end{array}$ & $\begin{array}{c}500 \mu \mathrm{m} \\
(\mathrm{mJy})\end{array}$ & $\begin{array}{l}S_{\text {cont }} \\
(\mathrm{mJy})\end{array}$ & $\begin{array}{c}T_{\exp }(\# \text { Antenna) } \\
\text { (seconds) }\end{array}$ \\
\hline GALEX 0959+0151 & $11.5 \pm 2.5$ & $12.4 \pm 2.1$ & $12.2 \pm 5.4$ & $0.5 \pm 4.0$ & $<2.8^{\mathrm{b}}$ & $<1.1^{\mathrm{b}}$ & $<0.0793^{\mathrm{c}}$ & $1255.87(27)$ \\
\hline GALEX $1000+0157$ & $<3.3^{\mathrm{b}}$ & $<2.1^{\mathrm{b}}$ & $9.4 \pm 5.4$ & $<4.0^{\mathrm{b}}$ & $<2.8^{\mathrm{b}}$ & $<1.1^{\mathrm{b}}$ & $<0.0484^{\mathrm{c}}$ & $9216.43(36)$ \\
\hline GALEX 1000+0201 & $7.3 \pm 4.2$ & $9.9 \pm 2.1$ & $6.2 \pm 5.4$ & $<4.0^{\mathrm{b}}$ & $<2.8^{\mathrm{b}}$ & $<1.1^{\mathrm{b}}$ & $<0.0658^{\mathrm{c}}$ & $9852.10(24)$ \\
\hline
\end{tabular}

Notes.

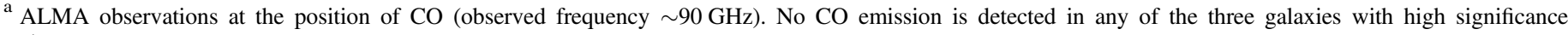
$\left(L_{\mathrm{CO}}^{\prime}<5.5 \times 10^{8} \mathrm{~K} \mathrm{~km} \mathrm{~s}^{-1} \mathrm{pc}^{2}\right.$ at $\left.3 \sigma\right)$.

b $1 \sigma$ upper limits on fluxes.

c $3 \sigma$ upper limits on fluxes.

indicate the KINGFISH (circles), DGS (squares), and GOALS (diamonds) samples. The metallicities are quoted in the Pilyugin \& Thuan (2005) calibration for a consistent comparison of the different samples. At intermediate to high total FIR luminosities $\left(\log \left(L_{\mathrm{FIR}} / L_{\odot}\right) \gtrsim 9\right)$, we recover the positive correlation between $L_{\mathrm{FIR}}$ and $T$ (and $T_{\text {peak }}$ ), similar to what was found in studies of luminous FIR galaxies (e.g., Dunne et al. 2000; Chapman et al. 2003; Magnelli et al. 2014). However, contrary to expected low temperatures at very low FIR luminosities, the FIR-faint and metal-poor dwarf galaxies from the DGS sample suggest increasing temperatures at $\log \left(L_{\mathrm{FIR}} / L_{\odot}\right) \lesssim 9$. This has not be seen in previous studies as those do not go this low in total FIR luminosity. It indicates that galaxies at low $L_{\mathrm{FIR}}$ and low metallicity as well as galaxies at high $L_{\text {FIR }}$ and high metallicity are characterized by a warm IR SED based on our local samples.

Figure 5 shows the (peak) temperature probability densities for our local samples. The KINGFISH sample, containing the most mature and metal-rich galaxies, shows the lowest distribution in $T$ and $T_{\text {peak }}$ of all of the samples (see also Figure 8 in Rémy-Ruyer et al. 2013). The dwarf galaxies in DGS, having significantly lower metallicities, show a $T$ distribution similar to the (U)LIRGs in the GOALS sample with sSFR $<2 \mathrm{Gyr}^{-1}$. Notably, galaxies with the highest sSFR ( $>2 \mathrm{Gyr}^{-1}$ in the GOALS sample) show an overall warmer IR SED, i.e., higher $T$. Taken at face value, this suggests that, in addition to a trend in metallicity, galaxies with higher sSFR have warmer IR SEDs. This is not entirely unexpected since sSFR is correlated with the total FIR luminosity via the SFR, which can introduce a positive correlation with $T$ as shown above. However, strictly speaking, sSFR is more similar to an FIR luminosity density and is therefore the preferred tool over the absolute $L_{\mathrm{FIR}}$ to compare galaxies at different redshifts.

The three low-metallicity analogues at $z \sim 0.3$ show $10-30 \mathrm{~K}\left(10-20 \mathrm{~K}\right.$ in $\left.T_{\text {peak }}\right)$ higher temperatures compared to the average of galaxies at similar metallicity, such as the dwarf galaxies (Figure 4). Hence, they seem to be outliers in the nearby galaxy samples. However, note that the analogues have relatively high sSFR (between 1.6 and $8.8 \mathrm{Gyr}^{-1}$ ) compared to these local samples, which is expected to boost their temperature further. Indeed, they show similar temperatures to the GOALS (U)LIRGs at similar sSFRs (Figure 5). We also note that the total FIR luminosities of the analogues do not match up with those of the local samples. Specifically, for their given metallicity, the analogues show $\sim 1-2$ orders of magnitude higher $L_{\mathrm{FIR}}$ compared to the $D G S$ galaxies. The same trends have been seen in other samples of analogue galaxies and are also indicated in our $z \sim 5.5$ sample $\left(\log \left(L_{\mathrm{FIR}} / L_{\odot}\right) \sim 10\right.$ at metallicities of $12+\log (\mathrm{O} / \mathrm{H}) \sim 7.5-8.0$ in the Pilyugin \& Thuan 2005 calibration, similar to the analogues-by definition). This simply means that galaxies with the same $L_{\mathrm{FIR}}$ but at different redshifts are not comparable, which is reasonable given their vastly different structure and stellar mass. Instead, the sSFR (a proxy for the FIR luminosity density) or, equivalently, the $\mathrm{H} \alpha$ surface density is a better parameter for comparing low- and high-redshift galaxies as well as to provide better analogue galaxies (Faisst 2016; Masters et al. 2016).

Summarizing, the local samples provide evidence that galaxies at low metallicity and high sSFRs are characterized by a warmer IR SED. The UV-analogue galaxies (extreme cases of low-redshift galaxies but with similar properties to high-redshift galaxies) strengthen these conclusions and suggest that high-redshift galaxies (characterized by low metallicity and high sSFRs) have a warmer IR SED compared to average galaxies at lower redshifts.

\subsection{Possible Reasons for a "Warm” IR SED}

In the previous section, we outlined evidence that galaxies with low metallicity and high sSFR show a "warmer" IR SED (i.e., higher $T$ and $T_{\text {peak }}$ ) compared to the average population. Ultimately, this may suggest that the average population of high-redshift galaxies (characterized by low metallicity and high sSFRs) have increased temperatures compared to typical galaxies at lower redshifts, which will be important to characterize their FIR properties, as will be shown later. In the following, we list the possible reasons that can cause a warm IR SED.

First of all, the presence of an AGN is one possible cause of a mid-IR excess, but known AGNs have been removed from the samples discussed above, and the location of our three analogues on the BPT diagram suggest no AGN component. A detailed analysis of X-ray emission from a dusty star-forming galaxy at $z \sim 5.6$ (SPT0346-52; $T=74 \mathrm{~K}, T_{\text {peak }}=43 \mathrm{~K}$ ) using Chandra also suggests that its FIR emission originates from vigorous star formation activity instead of an AGN (Ma et al. 2016). This is strengthened by a recent analysis of a sample of four "Green Peas" (low-redshift analogues of $z \sim 3$ galaxies) that arrives at the same conclusion (Lofthouse et al. 2017). However, the presence of an obscured AGN cannot be ruled out, and in fact, it can be easily missed by traditional selections as shown in the case of GN20 at $z \sim 4$ (Riechers et al. 2014) or other examples at lower redshifts (Pope et al. 2008; Coppin et al. 2010). This obscured AGN can even dominate the mid-IR 
Table 3

Fitted UV and Optical Properties of the Three Low-redshift Analogues

\begin{tabular}{|c|c|c|c|c|c|c|c|c|c|}
\hline Name & $\begin{array}{c}\mathrm{EW}(\mathrm{H} \alpha) \\
(\AA)\end{array}$ & {$[\mathrm{O} \mathrm{III}] / \mathrm{H} \alpha$} & {$[\mathrm{O} \mathrm{III]}] / \mathrm{H} \beta$} & {$[\mathrm{O} \mathrm{III}] /[\mathrm{O} \mathrm{II}]$} & $\log \left(M / M_{\odot}\right)$ & $\begin{array}{c}E(B-V) \\
(\mathrm{mag})\end{array}$ & $\log \left(L_{\mathrm{UV}} / L_{\odot}\right)$ & $\begin{array}{c}\beta^{\mathrm{a}} \\
\text { (UV cont. slope) }\end{array}$ & $\begin{array}{c}\mathrm{SFR}_{\mathrm{UV}+\mathrm{FIR}}{ }^{\mathrm{b}} \\
\left(M_{\odot} \mathrm{yr}^{-1}\right)\end{array}$ \\
\hline \multirow{3}{*}{$\begin{array}{l}\text { GALEX } 0959 \\
\quad+0151\end{array}$} & $452 \pm 14$ & $1.95 \pm 0.14$ & $5.56 \pm 0.41$ & $3.23 \pm 0.57$ & $9.04 \pm 0.17$ & $0.16 \pm 0.06$ & $10.12_{-0.08}^{+0.07}$ & $-1.80 \pm 0.46$ & 8.8 \\
\hline & $(333)^{c}$ & $(2.11)^{\mathrm{c}}$ & $(6.04)^{\mathrm{c}}$ & $4.11 \pm 0.72$ & $9.11 \pm 0.16$ & $0.18 \pm 0.07$ & $10.11_{-0.10}^{+0.08}$ & $-1.71 \pm 0.55$ & \\
\hline & & & & & & \multicolumn{2}{|c|}{$\beta$ from $G A L E X:$} & $-1.43_{-0.14}^{+0.24}$ & \\
\hline \multirow{3}{*}{$\begin{array}{l}\text { GALEX } 1000 \\
+0157\end{array}$} & $402 \pm 18$ & $2.00 \pm 0.11$ & $5.73 \pm 0.32$ & $3.39 \pm 0.56$ & $8.9 \pm 0.15$ & $0.07 \pm 0.04$ & $10.33_{-0.06}^{+0.05}$ & $-2.19 \pm 0.23$ & 4.9 \\
\hline & $(423)^{c}$ & $(1.96)^{\mathrm{c}}$ & $(5.61)^{\mathrm{c}}$ & $3.38 \pm 0.52$ & $8.89 \pm 0.14$ & $0.07 \pm 0.03$ & $10.33_{-0.06}^{+0.05}$ & $-2.20 \pm 0.22$ & \\
\hline & & & & & & \multicolumn{2}{|c|}{$\beta$ from GALEX: } & $-1.48_{-0.40}^{+0.87}$ & \\
\hline \multirow{3}{*}{$\begin{array}{l}\text { GALEX } 1000 \\
\quad+0201\end{array}$} & $150 \pm 13$ & $1.89 \pm 0.23$ & $5.41 \pm 0.66$ & $1.55 \pm 0.24$ & $9.55 \pm 0.14$ & $0.26 \pm 0.08$ & $10.24_{-0.16}^{+0.11}$ & $-1.52 \pm 0.70$ & 6.5 \\
\hline & $(195)^{c}$ & $(1.65)^{\mathrm{c}}$ & $(4.71)^{\mathrm{c}}$ & $1.48 \pm 0.09$ & $9.53 \pm 0.13$ & $0.26 \pm 0.07$ & $10.24_{-0.14}^{+0.10}$ & $-1.54 \pm 0.66$ & \\
\hline & & & & & & \multicolumn{2}{|c|}{$\beta$ from GALEX: } & $-1.86_{-0.14}^{+0.28}$ & \\
\hline
\end{tabular}

Notes.

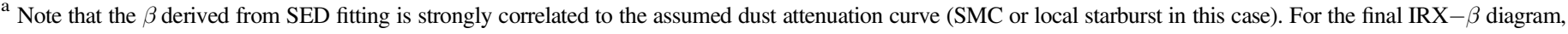
we therefore use the $\beta$ independently computed from the GALEX photometry, corrected by the Ly $\alpha$ emission contaminating the FUV filter.

${ }^{\mathrm{b}}$ UV measured at $1600 \AA$ and FIR from $L_{\mathrm{FIR}}$; see Table 4 .

c These parameters are fixed to the spectroscopic value during the SED fitting.

continuum but it is shown by these studies that it has a minor impact on the FIR part of the SED and therefore only a modest effect on the temperature.

We found evidence that galaxies with low metallicity as well as high sSFR have increased $T$. This suggests that there might be at least two mechanisms that cause such a warm IR SED. These might be working at the same time, especially in the lowmetallicity and high-sSFR analogues and likely also highredshift galaxies. On one hand, a high sSFR is indicative of a high density of intense UV radiation fields originating from young star-forming regions that are heating up the surrounding ISM. In the local universe, this might be the case for compact FIR-luminous galaxies such as the ULIRGS in GOALS (Figure 5). At high redshifts, however, this could generally be the case as these galaxies are very compact and reveal a higher population-averaged sSFR. This picture is generally supported by the detection of extended [C II] emission from the warm ISM around local LIRGs (Díaz-Santos et al. 2014) and in samples of high-redshift galaxies (Section 4.3). On the other hand, a likely explanation for the warm IR SEDs could be a lower optical depth in the ISM due to lower dust column densities at lower metallicities. As shown by Scoville \& Kwan (1976), the mid-IR shape of the SED is not only affected by the temperature but also by the mean optical depth of the ISM and the dust mass (see also Scoville 2013). In detail, for optically thin dust, the exponential mid-IR blackbody tail is modified to a power law, and the peak wavelength is shifted bluewards, both causing an increase in $T$ as well as $T_{\text {peak }}$. The same, but weaker, trend is expected for a decreasing dust mass. At the same time, harder UV radiation might be prevalent in lowmetallicity environments with massive stars caused by less efficient ISM cooling. Interestingly, Prestwich et al. (2013) also suggest a higher number of X-ray sources at lower metallicity that could be responsible for heating the ISM. In the local universe, these effects are expected in metal-poor and FIR-faint dwarf galaxies (Figure 4). At high redshifts, this could be commonly the case as these galaxies have metallicities significantly below solar.

\section{The IRX $-\beta$ Diagnostic Tool}

The general idea behind the IRX $-\beta$ correlation is that dust in the ISM and circumgalactic medium around galaxies absorbs the blue light from young $\mathrm{O}$ and $\mathrm{B}$ stars and re-emits it in the FIR. Therefore, the ratio of FIR to UV luminosity $\left(L_{\mathrm{FIR}} / L_{\mathrm{UV}}=\right.$ IRX) is sensitive to the total dust mass and the geometry of dust grains. On the other hand, the UV color (measured by the UV continuum spectral slope $\beta$ ) is sensitive to the line-of sight opacity (e.g., Bouwens et al. 2012). ${ }^{16}$ The IRX $-\beta$ diagram combines these two measures and can therefore be used for studying the dust and gas properties and distribution in galaxies with a minimal amount of input.

\subsection{Analytical Models to Explain IRX - $\beta$ in Typical Local to Intermediate-redshift Galaxies}

Charlot \& Fall (2000; see also Kong et al. 2004) describe a simple analytical model for the absorption of starlight in the ISM of galaxies, which can explain the location of typical galaxies on the IRX $-\beta$ diagram up to $z \sim 3-4$ where detailed data exist. The model assumes dust in thermal equilibrium that is distributed in the ambient ISM in one of the following three geometrical configurations: (i) a uniform foreground screen characterized by a single optical depth $\tau_{\lambda}^{s c}$, (ii) a "mixed slab" characterized by a uniform mixture of dust and stars and optical depth $\tau_{\lambda}^{s l}$, and (iii) a Poissonian distribution of discrete clouds characterized by an optical depth per cloud $\left(\tau_{\lambda}^{c}\right)$ and the average number of clouds mixed with stars along the line of sight to the observer $(\bar{n})$. Moreover, the models assume that the young stars are enshrouded in birth clouds that dissolve after a finite lifetime of $t_{\mathrm{bc}}=10 \mathrm{Myr}$ (see also Vallini et al. 2017). The total transmission for UV light, namely, the product of the transmission function through the birth clouds and the ambient

\footnotetext{
$\overline{16}$ Note that $\beta$ also depends on the age of the stellar population, the SFH, and metallicity. However, for young stellar populations, as is the case in young starforming galaxies especially at high redshifts, the intrinsic UV slope is expected to be invariant (Scoville et al. 2015), and hence we can assume $\beta$ to be a good proxy for the line-of-sight opacity.
} 
Table 4

FIR Properties of the Three Low- $z$ Analogues and High- $z$ Galaxies from the Literature

\begin{tabular}{|c|c|c|c|c|c|c|}
\hline Name & $z$ & $\begin{array}{c}\alpha \\
\text { (MIR power-law slope) }\end{array}$ & $\begin{array}{c}\beta_{\mathrm{IR}} \\
\text { (emissivity) }\end{array}$ & $\begin{array}{r}T^{\mathrm{a}} \\
(\mathrm{K})\end{array}$ & $\begin{array}{r}T_{\text {peak }}{ }^{\mathrm{b}} \\
(\mathrm{K})\end{array}$ & $\log \left(L_{\mathrm{FIR}} / L_{\odot}\right)^{\mathrm{c}}$ \\
\hline \multicolumn{7}{|c|}{ Low-redshift analogues (This work) } \\
\hline GALEX $1000+0157^{\mathrm{d}}$ & 0.2647 & $2.42_{-0.61}^{+0.47}$ & $1.80_{-1.47}^{+3.00}$ & $72_{-46}^{+35}$ & $43_{-26}^{+18}$ & $10.32_{-0.37}^{+0.15}$ \\
\hline GALEX 1000+0201 & 0.2653 & $2.47_{-0.11}^{+0.09}$ & $1.18_{-1.28}^{+3.16}$ & $75_{-5}^{+8}$ & $43_{-0}^{+5}$ & $10.68_{-0.09}^{+0.06}$ \\
\hline Stack & $\ldots$ & $2.12_{-0.63}^{+1.25}$ & $2.88_{-2.36}^{+12.87}$ & $84_{-17}^{+9}$ & $48_{-5}^{+6}$ & $10.68_{-0.04}^{+0.03}$ \\
\hline \multicolumn{7}{|c|}{ (Parameters derived for $1.5<\alpha<2.5,1.0<\beta_{\mathrm{IR}}<2.0,60 \mathrm{~K}<T<90 \mathrm{~K}\left[35 \mathrm{~K}<T_{\text {peak }}<50 \mathrm{~K}\right]$ ) } \\
\hline HZ1 & 5.6885 & $\ldots$ & $\ldots$ & $\ldots$ & $\ldots$ & $<10.96_{-0.25}^{+0.22}$ \\
\hline HZ2 & 5.6697 & $\ldots$ & $\ldots$ & $\ldots$ & $\ldots$ & $<10.95_{-0.25}^{+0.22}$ \\
\hline HZ3 & 5.5416 & $\ldots$ & $\ldots$ & $\ldots$ & $\ldots$ & $<11.18_{-0.25}^{+0.22}$ \\
\hline HZ4 & 5.5440 & $\ldots$ & $\ldots$ & $\ldots$ & $\ldots$ & $11.78_{-0.25}^{+0.22}$ \\
\hline HZ6b & & $\ldots$ & $\ldots$ & $\ldots$ & $\ldots$ & $11.52_{-0.25}^{+0.22}$ \\
\hline HZ6c & & $\ldots$ & $\ldots$ & $\ldots$ & $\ldots$ & $11.44_{-0.25}^{+0.22}$ \\
\hline $\mathrm{HZ7}$ & 5.2532 & $\ldots$ & $\ldots$ & $\ldots$ & $\ldots$ & $<10.99_{-0.25}^{+0.22}$ \\
\hline HZ8 & 5.1533 & $\ldots$ & $\ldots$ & $\ldots$ & $\ldots$ & $<10.90_{-0.25}^{+0.22}$ \\
\hline HZ8W & & $\ldots$ & $\ldots$ & $\ldots$ & $\ldots$ & $<10.90_{-0.25}^{+0.22}$ \\
\hline HZ9 & 5.5410 & $\ldots$ & $\ldots$ & $\ldots$ & $\ldots$ & $12.18_{-0.25}^{+0.22}$ \\
\hline HZ10 & 5.6566 & $\ldots$ & $\ldots$ & $\ldots$ & $\ldots$ & $12.58_{-0.25}^{+0.22}$ \\
\hline$\cdot \mathrm{HZ1OW}^{\mathrm{h}}$ & & $\ldots$ & $\ldots$ & $\ldots$ & $\ldots$ & $\left(12.28_{-0.2}^{+0.2}\right.$ \\
\hline
\end{tabular}

Temperature constraints on high- $z$ galaxies from Pavesi et al. (2016)

\begin{tabular}{lcccrr}
\hline & (Parameters derived for $1.2<\beta_{\mathrm{IR}}<2.2,10 \mathrm{~K}<T<100 \mathrm{~K}\left[5 \mathrm{~K}<T_{\text {peak }}<60 \mathrm{~K}\right], \alpha$ is unconstrained) \\
$\mathrm{HZ6}$ (all) & 5.2928 & $\cdots$ & $\cdots$ & $60_{-27}^{+35}$ & $35_{-16}^{+20}$ \\
$\mathrm{HZ10}$ & 5.6566 & $\cdots$ & $\cdots$ & $36_{-10}^{+25}$ & $25_{-7}^{+18}$ \\
\hline
\end{tabular}

Lensed high- $z$ Herschel-detected $1.5<z<3.0$ galaxies (Sklias et al. 2014)

\begin{tabular}{|c|c|c|c|c|c|c|}
\hline \multicolumn{7}{|c|}{ (Modified blackbody with fixed $\beta_{\mathrm{IR}}=1.5$, corrected for magnification) } \\
\hline $\mathrm{A} 68 / \mathrm{C} 0$ & 1.59 & $\ldots$ & $\ldots$ & $\ldots$ & $34.5_{-1}^{+1}$ & 11.06 \\
\hline $\mathrm{A} 68 / \mathrm{h} 7$ & 2.15 & $\ldots$ & $\ldots$ & $\ldots$ & $43.4_{-1}^{+1}$ & 12.26 \\
\hline A68/HLS115 & 1.59 & $\ldots$ & $\ldots$ & $\ldots$ & $37.5_{-1}^{+1}$ & 11.53 \\
\hline $\mathrm{A} 68 / \mathrm{nn} 4$ & 3.19 & $\ldots$ & $\ldots$ & $\ldots$ & $54.9_{-1}^{+1}$ & 12.83 \\
\hline MACS0451 north & 2.01 & $\ldots$ & $\ldots$ & $\ldots$ & $49.2_{-1}^{+1}$ & 10.87 \\
\hline MACS0451 full arc & $" 1$ & $\ldots$ & $\ldots$ & $\ldots$ & $50-80$ & 11.27 \\
\hline \multicolumn{7}{|c|}{ Lensed high- $z$ DSFGs (Strandet et al. 2016) } \\
\hline \multicolumn{7}{|c|}{ (Refitted by our method with photometry from Strandet et al. 2016) } \\
\hline SPT2319- $55^{\mathrm{i}}$ & 5.2929 & $1.73_{-1.17}^{+53.19}$ & $2.46_{-0.16}^{+0.17}$ & $59_{-7}^{+5}$ & $34_{-1}^{+4}$ & $<13.79_{-0.17}^{+0.40}$ \\
\hline SPT2353-50 & 5.5760 & $0.91_{-0.59}^{+3.28}$ & $2.60_{-0.18}^{+0.18}$ & $61_{-6}^{+6}$ & $38_{-4}^{+1}$ & $<14.02_{-0.28}^{+0.36}$ \\
\hline SPT0346-52 ${ }^{\mathrm{i}}$ & 5.6559 & $2.07_{-0.72}^{+5.36}$ & $2.52_{-0.66}^{+2.16}$ & $74_{-3}^{+3}$ & $43_{-5}^{+1}$ & $13.52_{-0.23}^{+0.160}$ \\
\hline SPT2351-57 & 5.8110 & $3.12_{-2.50}^{+17.55}$ & $2.41_{-0.16}^{+0.17}$ & $80_{-7}^{+5}$ & $48_{-5}^{+1}$ & $<13.92_{-0.08}^{+0.41}$ \\
\hline \multicolumn{7}{|c|}{ Intense high- $z$ starburst $A z T E C$-3 (Riechers et al. (2014) } \\
\hline AzTEC- 3 & 5.2988 & $6.17_{-2.60}^{+2.73}$ & $2.16_{-0.27}^{+0.27}$ & $88_{-10}^{+10}$ & $51_{-6}^{+6}$ & $13.34_{-0.10}^{+0.08}$ \\
\hline \multicolumn{7}{|c|}{ High- $z$ galaxies from Willott et al. (2015) } \\
\hline \multicolumn{7}{|c|}{ (Parameters derived for $1.5<\alpha<2.5,1.0<\beta_{\mathrm{IR}}<2.0,60 \mathrm{~K}<T<90 \mathrm{~K}\left[35 \mathrm{~K}<T_{\text {peak }}<50 \mathrm{~K}\right]$ ) } \\
\hline CLM1 & 6.1657 & $\ldots$ & $\ldots$ & $\ldots$ & $\ldots$ & $11.18_{-0.25}^{+0.22}$ \\
\hline WMH5 & 6.0695 & $\ldots$ & $\ldots$ & $\ldots$ & $\ldots$ & $11.87_{-0.25}^{+0.22}$ \\
\hline \multicolumn{7}{|c|}{ Candidate [C II]-detected high- $z$ galaxies from Aravena et al. (2016; blind search) } \\
\hline IDX25 & $\begin{array}{c}\text { (Paramete } \\
6.357\end{array}$ & $\begin{array}{l}\alpha<2.5,1.0 \\
\ldots\end{array}$ & $\begin{array}{c}0 \mathrm{~K}<T< \\
\ldots\end{array}$ & $\begin{array}{l}<T_{\mathrm{p}} \\
\ldots\end{array}$ & ]) & $<11.16_{-0.25}^{+0.22}$ \\
\hline
\end{tabular}


Table 4

(Continued)

\begin{tabular}{|c|c|c|c|c|c|c|}
\hline Name & $z$ & $\begin{array}{c}\alpha \\
\text { (MIR power-law slope) }\end{array}$ & $\begin{array}{c}\beta_{\mathrm{IR}} \\
\text { (emissivity) }\end{array}$ & $\begin{array}{r}T^{\mathrm{a}} \\
(\mathrm{K})\end{array}$ & $\begin{array}{r}T_{\text {peak }}{ }^{\mathrm{b}} \\
(\mathrm{K})\end{array}$ & $\log \left(L_{\mathrm{FIR}} / L_{\odot}\right)^{\mathrm{c}}$ \\
\hline IDX34 & 7.491 & $\ldots$ & $\ldots$ & $\ldots$ & $\ldots$ & $<11.66_{-0.25}^{+0.22}$ \\
\hline ID02 & 7.914 & $\ldots$ & $\ldots$ & $\ldots$ & $\ldots$ & $<11.43_{-0.25}^{+0.22}$ \\
\hline ID04 & 6.867 & $\ldots$ & $\ldots$ & $\ldots$ & $\ldots$ & $11.53_{-0.25}^{+0.22}$ \\
\hline ID09 & 6.024 & $\ldots$ & $\ldots$ & $\ldots$ & $\ldots$ & $<11.15_{-0.25}^{+0.22}$ \\
\hline ID14 & 6.751 & $\ldots$ & $\ldots$ & $\ldots$ & $\ldots$ & $<11.22_{-0.25}^{+0.22}$ \\
\hline ID31 & 7.494 & $\ldots$ & $\ldots$ & $\ldots$ & $\ldots$ & $11.14_{-0.25}^{+0.22}$ \\
\hline ID38 & 6.593 & $\ldots$ & $\ldots$ & $\ldots$ & $\ldots$ & $<11.20_{-0.25}^{+0.22}$ \\
\hline ID41 & 6.346 & $\ldots$ & $\ldots$ & $\ldots$ & $\ldots$ & $<11.20_{-0.25}^{+0.22}$ \\
\hline ID44 & 7.360 & $\ldots$ & $\ldots$ & $\ldots$ & $\ldots$ & $<11.25_{-0.25}^{+0.22}$ \\
\hline ID49 & 6.051 & $\ldots$ & $\ldots$ & $\ldots$ & $\ldots$ & $<11.13_{-0.25}^{+0.22}$ \\
\hline ID52 & 6.018 & $\ldots$ & $\ldots$ & $\ldots$ & $\ldots$ & $<11.37_{-0.25}^{+0.22}$ \\
\hline
\end{tabular}

Notes.

${ }^{\text {a }}$ Luminosity-weighted temperature as in Casey (2012).

b Temperature measured from the wavelength of the peak flux emission via Wien's displacement law.

${ }^{c}$ The total FIR luminosity is integrated between rest-frame 3-1100 $\mu \mathrm{m}$. If necessary, the values are converted into this integration interval.

${ }^{\mathrm{d}}$ This source is IR faint, and therefore these estimates are mostly based on limits causing the large uncertainties.

${ }^{\mathrm{e}} L_{\mathrm{FIR}}$ has been corrected for magnification using $\mu=5.4$ (Strandet et al. 2016).

${ }^{\mathrm{f}}$ This object is a low-luminosity quasar.

$\mathrm{g}$ This object is called LBG-1 in Riechers et al. (2014) and Pavesi et al. (2016)

h Assuming half of the FIR luminosity of HZ10.

${ }^{\mathrm{i}}$ No constraints blueward of rest-frame $40 \mu \mathrm{m}$ are available for these sources, causing the estimate for $\alpha$ to be very uncertain.

${ }^{\mathrm{j}}$ The magnification for these sources is not known. Therefore, the $L_{\mathrm{FIR}}$ has not been demagnified in these cases.

ISM for $t<t_{\mathrm{bc}}$, and the transmission function alone through the ISM otherwise, is therefore time varying.

These different models successfully explain the typical galaxy population on the IRX $-\beta$ diagram. Specifically, Charlot \& Fall (2000) find that the model featuring discrete clouds in the ISM best represents the IRX and UV color distribution of typical local starburst galaxies by assuming a simple power-law relation $\tau_{\lambda}^{c}=\tau_{V}^{c}(\lambda / 5500 \AA)^{-1.3}$ for the wavelength dependence of the optical depth of the ambient ISM. Such simple forms for $\tau_{\lambda}$ with a logarithmic slope of -1.3 are expected from various detailed measurements of the dust extinction curves in the Milky Way galaxy (Seaton 1979), the Large Magellanic Cloud (Fitzpatrick 1986), local starburst galaxies (Calzetti et al. 2000), and even normal star-forming galaxies at $z=2-4$ (Scoville et al. 2015). For the birth clouds, a similar optical depth is assumed with $\tau_{\lambda}^{b c}=0.7(\lambda / 5500 \AA)^{-0.7}$, but we note that the exact relation does not impact the following conclusions. We show such a model in Figure 6 for a stellar population of $300 \mathrm{Myr}$ and $\bar{n}$ running from 1 to 10 with $\tau_{V}^{c}=0.5$ (orange circles) and $\tau_{V}^{c}=0.1$ (green squares). ${ }^{17}$

This simple model does successfully explain galaxies with dust properties similar to the local starbursts, but it fails to reproduce galaxies with lower IRX values at a fixed UV color even for the lowest cloud optical depths (i.e., $\tau_{V}^{c}$ ). This part of the parameter space can be covered within this model by steepening the dust extinction curve, i.e., changing the form of the wavelengthdependent optical depth $\tau_{\lambda}^{c}$. The blue triangles in Figure 6 show the cloud model with the more complex $\tau_{\lambda}^{c}$ of the metal-poor SMC parameterized by a threefold broken power law (e.g.,

\footnotetext{
17 We assume a simple stellar population published with GALAXEV (Bruzual \& Charlot 2003) for half-solar metallicity, a constant SFH, and a Chabrier (2003) IMF.
}

Prevot et al. 1984) and assuming $\bar{n}=3$ and $\tau_{V}^{c}$ up to 0.05 . With this modification, the model can reproduce galaxies with lower IRX values at a given UV color for SMC-like dust. At the same time, this shows how we can study the dust properties of galaxies with this very simple model of dust absorption and a minimal amount of data; the location of galaxies on the IRX $-\beta$ diagram tells us about their total amount of dust, the optical depth of their clouds in the ISM, and the wavelength dependence of their dust attenuation curve. These properties ultimately depend on other physical properties of the galaxies, for example, their metallicity as shown in the case of SMC-like dust. We note that in the recent study by Popping et al. (2017b), the low IRX values and red UV continuum slopes can also be explained by old and dust-poor stellar populations (see also Howell et al. 2010). Observations are necessary in order to verify or disproof this solution for a given sample at high redshift.

\subsection{Correlation between the Location on the IRX- $\beta$ Diagram and Physical Properties}

The IRX $-\beta$ diagram has also been studied thoroughly from an observational point of view, and it is found that the galaxy properties change significantly as a function of position on this diagram (e.g., Howell et al. 2010; Reddy et al. 2010; Boquien et al. 2012; Casey et al. 2014; Álvarez-Márquez et al. 2016).

For example, more mature, metal-rich, and dust-rich galaxies are expected to show high values of IRX, and thus they are found in the upper part of the IRX $-\beta$ diagram. Such galaxies are pictured as compact, dust-enshrouded, IR-luminous starforming systems that have likely experienced a recent starburst after a merger event (e.g., Larson et al. 2016). The blue colors of some of these galaxies can be explained by a patchy dust screen (Casey et al. 2014) or tidally stripped unobscured young 

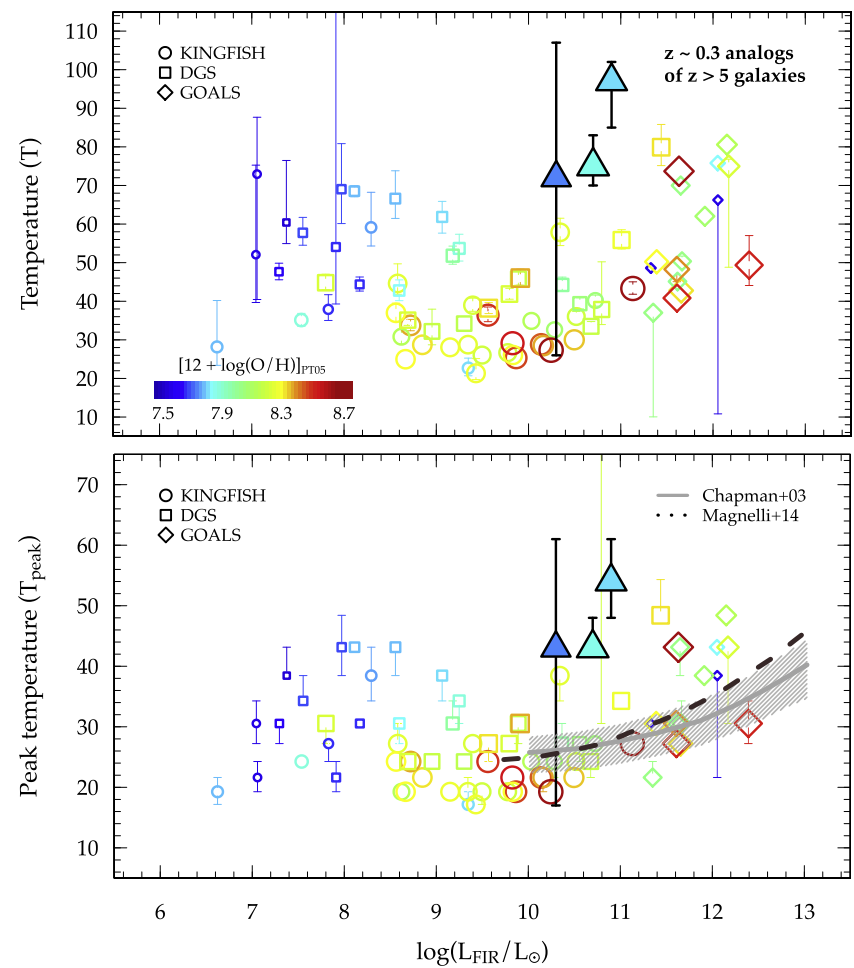

Figure 4. $L_{\mathrm{FIR}}$ Vs. $T$ relation (top) and $L_{\mathrm{FIR}}$ Vs. $T_{\text {peak }}$ relation (bottom) colored by metallicity based on the KINGFISH (circles), GOALS (squares), and DGS (diamonds) local galaxy samples. There are indications that $T$ and $T_{\text {peak }}$ are enhanced in metal-poor galaxies. The correlations by Chapman et al. (2003; $z \sim 0$, gray hatched region) and Magnelli et al. (2014; $0.2<z<0.5$, dashed black line) are indicated for $T_{\text {peak. }}$. Our three $z \sim 0.3$ analogues are shown as big symbols color-coded by their metallicity. We use the metallicity calibration of Pilyugin \& Thuan (2005) here for a consistent comparison. These local relations indicate that high-redshift galaxies (characterized by high sSFR and low metallicity) have a "warmer" IR SED than average galaxies at lower redshifts.

stars, or a small spatially unresolved and unobscured starforming satellite as suggested by some IR-luminous $\left(\log \left(L_{\text {FIR }} / L_{\odot}\right)>12\right)$ local galaxies (e.g., Howell et al. 2010). An alternative scenario that does not involve starbursts or mergers is predicted by hydrodynamical simulations. These suggest that isolated normal main-sequence galaxies with $\log \left(L_{\mathrm{FIR}} / L_{\odot}\right)<12$ can populate the upper-left part of the IRX $-\beta$ diagram naturally due to their high gas fractions and high metal enrichment (e.g., Hopkins et al. 2010; Safarzadeh et al. 2017). The observed increase in gas fraction toward higher redshifts (Tacconi et al. 2010; Genzel et al. 2015) would make such a configuration likely for the most metal-rich galaxies in the early universe.

On the other hand, younger, metal-poor, and dust-poor galaxies are expected to show low values of IRX and blue UV colors, and thus populate the lower parts of the IRX $-\beta$ diagram (see Figure 7). They could be characterized by a steeper dust attenuation curve as seen in the metal-poor SMC, which causes a flatter IRX $-\beta$ relation for these galaxies (see Section 3.1 and discussion in Reddy et al. 2010). Galaxies in the early universe are expected to be young, dust poor, and less metal enriched on average (Maiolino et al. 2008; Mannucci et al. 2010; Feldmann 2015; Bian et al. 2017; Faisst et al. 2016b; Popping et al. 2016), and thus are expected to reside on the lower parts of the IRX $-\beta$ diagram as predicted by the Charlot \& Fall (2000) model with an SMC-like dust attenuation curve. This has been directly observed in some FIR-detected $z \sim 3$ galaxies (e.g., Baker et al. 2001; Siana et al. 2009; Reddy et al. 2010), and there are also hints of this in our small sample of $z \sim 0.3$ analogues; the analogues GALEX 0959+0151 and GALEX 1000+0201 (both sitting on the IRX $-\beta$ relation of local starbursts) have metallicities $\sim 0.2$ dex higher than that ofGALEX $1000+0157$, which is located on the relation of the SMC. From the correlation with metallicity, we would also expect a correlation between position on the IRX - $\beta$ diagram and IR SED shape (Section 2.3). As detailed in Section 2.4, a warm IR SED can be due to heating of the ISM by a high amount of star formation or due to a low dust optical depth in the ISM as shown by Scoville \& Kwan (1976). The latter is supported by the simple dust model discussed in Section 3.1, which predicts a very low optical depth $\left(\tau_{V}^{c}<0.05\right)$ for the clouds in galaxies close to the SMC relation.

Extrapolating our knowledge gained from our low-redshift samples, we expect galaxies in the early universe to show warm SEDs as well as high rates of star formation, which will make them appear close to the SMC relation.

\section{The IRX $-\beta$ Diagram at $z \sim 5.5$}

The advent of ALMA has allowed us to begin populating the IRX $-\beta$ diagram at $z>5$ (e.g., Ota et al. 2014; Capak et al. 2015; Maiolino et al. 2015; Aravena et al. 2016; Bouwens et al. 2016). C15 presents today's largest and most representative sample of normal main-sequence $z \sim 5-6$ galaxies with detected [C II] emission and continuum detections or limits at $158 \mu \mathrm{m}$ along with robust UV measurements (Barišić et al. 2017). From the reasoning in Sections 3.1 and 3.2, we expect that most of the high-redshift galaxies reside in the lower part of the IRX $-\beta$ diagram, close to the relation of the SMC. However, we also expect a large variation in the metallicity and star-forming properties of high- $z$ galaxies (e.g., Steinhardt et al. 2014; Faisst et al. 2016b; Barišić et al. 2017). We therefore also expect a significant scatter in IRX $-\beta$.

The C15 sample consists of nine star-forming Lyman Break galaxies at 1-4 $L_{\mathrm{UV}}^{*}$ (the characteristic knee of the galaxy luminosity function) and one low-luminosity quasar (HZ5) at redshifts $5.1<z<5.7$. All galaxies are located in the 2 squaredegree COSMOS field and therefore benefit from a multitude of ground- and space-based photometric and spectroscopic data.

The galaxies are spectroscopically selected via their rest-frame UV absorption features from spectra obtained with the Deep Extragalactic Imaging Multi-Object Spectrograph (DEIMOS; Faber et al. 2003) and represent a large range in stellar masses, SFRs, and UV luminosities at $z \sim 5.5$. A detailed analysis of their DEIMOS optical spectra and Spitzer photometry properties suggests gas-phase metallicities around $12+\log (\mathrm{O} / \mathrm{H}) \sim 8.5$, which is representative of the average metallicity of $\log \left(M / M_{\odot}\right) \sim 10$ star-forming galaxies at $z \sim 5$ (Ando et al. 2007; Faisst et al. 2016b).

All galaxies were observed with ALMA at rest-frame $\sim 150 \mu \mathrm{m}$ and are detected in [C II] emission at $158 \mu \mathrm{m}$ (Riechers et al. 2014; Capak et al. 2015). Four of them (HZ4, HZ6, HZ9, and HZ10) are detected in continuum. Furthermore, HZ6 and HZ10 have been detected in [N II] line emission and continuum at $205 \mu \mathrm{m}$ (Pavesi et al. 2016). Both the low-luminosity quasar (HZ5) as well as HZ8 have a [C II]-detected companion (HZ5a and HZ8W) at their corresponding redshifts without significant detection in groundbased near-IR imaging data. HZ6 is a system of three galaxies $\left(\mathrm{HZ6a}, \mathrm{HZ6b}\right.$, and HZ6c) ${ }^{18}$ with detected [C II] emission and

\footnotetext{
$\overline{18}$ These are called LBG-1a through LBG-1c in Riechers et al. (2014) and Pavesi et al. (2016).
} 

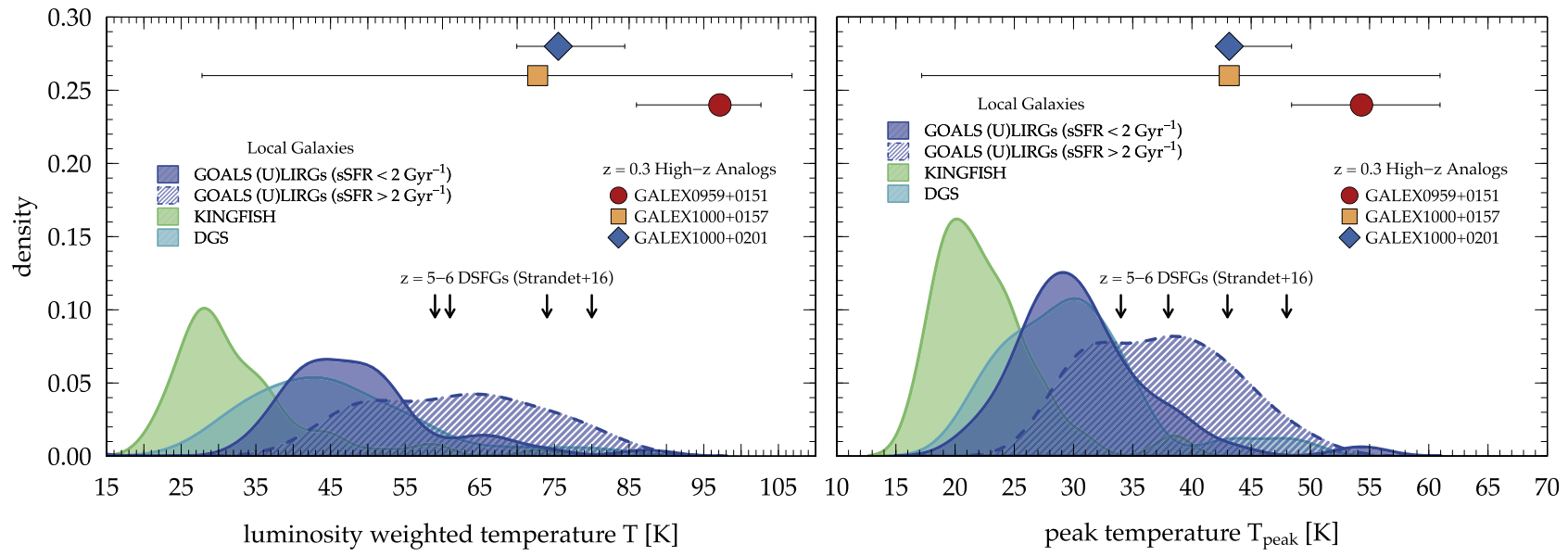

Figure 5. Distribution of the luminosity-weighted temperature ( $T$, left) and peak temperature ( $T_{\text {peak }}$, right) for nearby KINGFISH (green), GOALS (blue), and DGS (cyan) galaxies. The GOALS sample is split into galaxies with high $\left(>2 \mathrm{Gyr}^{-1}\right)$ and low $\left(<2 \mathrm{Gyr}^{-1}\right)$ sSFR to emphasize the correlation between the peak wavelength and sSFR. The high-sSFR local galaxies show similar temperatures to our $z \sim 0.3$ analogues (symbols) and the $z \sim 5-6$ lensed DSFGs from Strandet et al. (2016).

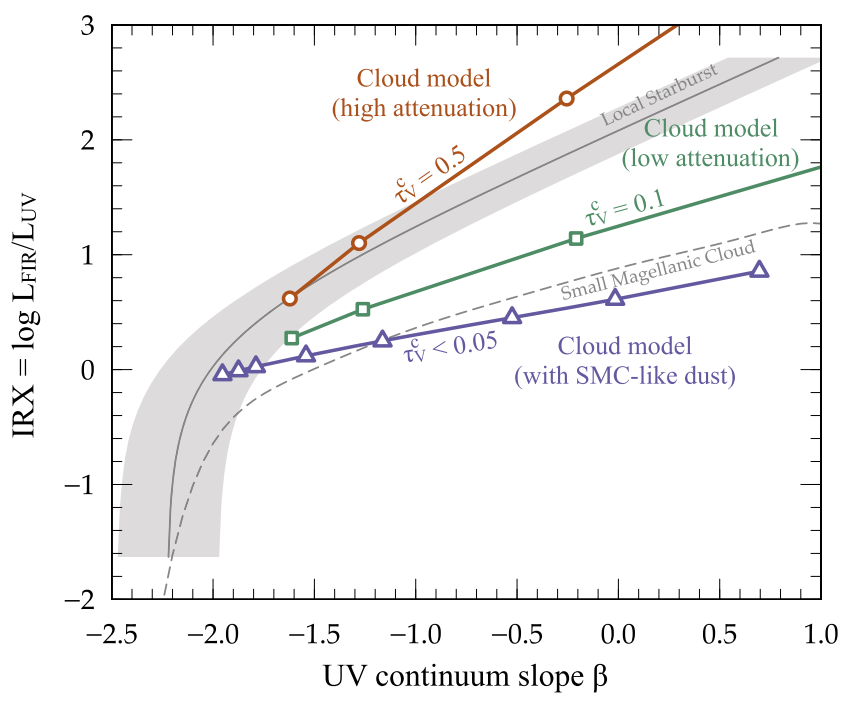

Figure 6. Different models for the absorption of starlight by dust in galaxies from Charlot \& Fall (2000). The line with orange circles and the line with green squares show the models for a Poissonian distribution of on average 1 to 10 clouds along the line of sight embedded in the ambient ISM with a wavelengthdependent opacity $\tau(\lambda) \propto \lambda^{-1.3}$ and $\tau_{V}^{c}=0.5$ and 0.1 , respectively. The line with blue solid triangles shows the same model as the steeper $\tau(\lambda)$ relation of the SMC for $\tau_{V}^{c}<0.05$.

$158 \mu \mathrm{m}$ continuum between HZ6a and HZ6b. HZ10 has a lowluminosity companion (HZ10W) that is, however, not spectroscopically confirmed in either optical or FIR lines.

All UV properties (morphology, luminosity, UV color) were accurately derived from deep near-IR imaging recently obtained by HST and are explained in detail in Barišić et al. (2017). Table 5 provides a summary of the UV and IR properties of the $\mathrm{C} 15$ sample.

\subsection{FIR Luminosity of High-z Galaxies}

The IR part of the SED is essential for studying the dust properties of galaxies. Although ALMA provides us with the necessary tools to observe the FIR part of the electromagnetic spectrum in high-redshift galaxies, it is very poorly constrained in normal (i.e., typical main-sequence) high-redshift galaxies due to their faintness, which makes such observations time

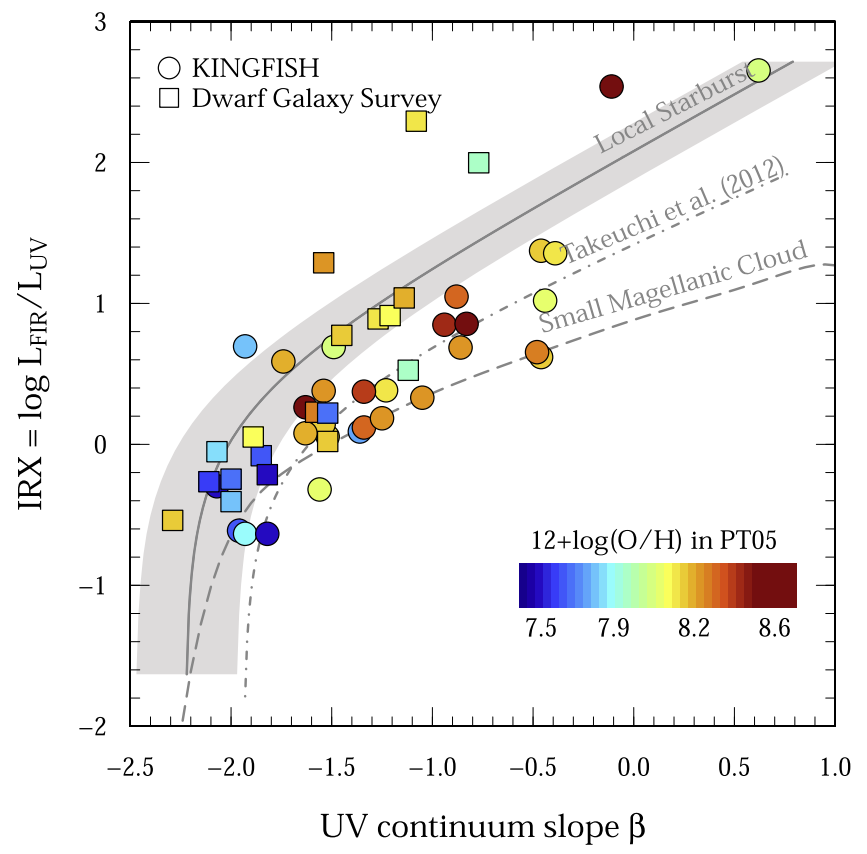

Figure 7. Correlation between metallicity and position on the IRX $-\beta$ diagram based on nearby galaxies from the KINGFISH and DGS samples. The lowmetallicity dwarf galaxies are preferentially located at blue UV spectral slopes and low IRX values, suggesting that galaxies at high redshift are located at similar locations.

consuming (see Appendix). Although good wavelength coverage in the FIR exists for a handful of bright submillimeter galaxies or FIR-luminous lensed systems at $z>5$ (Riechers et al. 2013, 2014; Strandet et al. 2016), only one continuum data point at rest-frame $150 \mu \mathrm{m}$ (close to the [C II] line) is commonly measured for typical main-sequence galaxies at these redshifts.

In particular, the total FIR luminosity $\left(L_{\mathrm{FIR}}\right)$, one of the most important parameters for constraining the location of galaxies on the IRX $-\beta$ diagram, is uncertain by a factor of $>3$ because of the poorly constrained IR SED (e.g., Schaerer et al. 2015). The current lack of accurate studies of the IR SEDs at high redshifts makes it necessary to assume an SED shape for these 
Table 5

Summary of the UV and FIR Properties of the C15 Galaxy Sample from Capak et al. (2015) and Barišić et al. (2017)

\begin{tabular}{|c|c|c|c|c|c|}
\hline Name & $\mathrm{z}$ & $\begin{array}{c}\beta \\
\text { (UV Spectral Slope) }\end{array}$ & $\log \left(L_{\mathrm{UV}} / L_{\odot}\right)$ & $\log \left(L_{[\mathrm{C} \text { п] }} / L_{\odot}\right)$ & $158 \mu \mathrm{m}$ Continuum Flux \\
\hline$\overline{\mathrm{HZ} 1}$ & 5.690 & $-1.92_{-0.11}^{+0.14}$ & $11.21 \pm 0.01$ & $8.40 \pm 0.32$ & $<30$ \\
\hline HZ2 & 5.670 & $-1.82_{-0.10}^{+0.10}$ & $11.15 \pm 0.01$ & $8.56 \pm 0.41$ & $<29$ \\
\hline $\mathrm{HZ3}$ & 5.546 & $-1.72_{-0.15}^{+0.12}$ & $11.08 \pm 0.01$ & $8.67 \pm 0.28$ & $<51$ \\
\hline $\mathrm{HZ} 4$ & 5.540 & $-2.06_{-0.15}^{+0.13}$ & $11.28 \pm 0.01$ & $8.98 \pm 0.22$ & $202 \pm 47$ \\
\hline HZ5 & 5.310 & $-1.01_{-0.12}^{+0.06}$ & $11.45 \pm 0.004$ & $<7.20$ & $<32$ \\
\hline . HZ5a & & ... & $<10.37$ & $8.15 \pm 0.27$ & $<32$ \\
\hline HZ6 (all) & 5.290 & $-1.14_{-0.14}^{+0.12}$ & $11.47 \pm 0.01$ & $9.23 \pm 0.04$ & $220 \pm 36$ \\
\hline - HZ6a & & $-0.59_{-1.12}^{+1.05}$ & $11.11 \pm 0.07$ & $8.32 \pm 0.06$ & $30 \pm 20$ \\
\hline · HZ6b & & $-1.50_{-1.22}^{+1.05}$ & $11.00 \pm 0.07$ & $8.81 \pm 0.02$ & $120 \pm 20$ \\
\hline . HZ6c & & $-1.30_{-0.37}^{+0.51}$ & $10.81 \pm 0.07$ & $8.65 \pm 0.03$ & $100 \pm 20$ \\
\hline $\mathrm{HZ7}$ & 5.250 & $-1.39_{-0.17}^{+0.15}$ & $11.05 \pm 0.02$ & $8.74 \pm 0.24$ & $<36$ \\
\hline HZ8 & 5.148 & $-1.42_{-0.18}^{+0.19}$ & $11.04 \pm 0.02$ & $8.41 \pm 0.18$ & $<30$ \\
\hline - HZ8W & & $-0.10_{-0.29}^{+0.29}$ & $10.57 \pm 0.04$ & $8.31 \pm 0.23$ & $<30$ \\
\hline HZ9 & 5.548 & $-1.59_{-0.23}^{+0.22}$ & $10.95 \pm 0.02$ & $9.21 \pm 0.09$ & $516 \pm 42$ \\
\hline HZ10 & 5.659 & $-1.92_{-0.17}^{+0.24}$ & $11.14 \pm 0.02$ & $9.13 \pm 0.13$ & $1261 \pm 44$ \\
\hline . $\mathrm{HZ} 10 \mathrm{~W}^{\mathrm{a}}$ & & $-1.47_{-0.44}^{+0.77}$ & $10.23 \pm 0.05$ & $(8.83 \pm 0.13)$ & $(630 \pm 44)$ \\
\hline
\end{tabular}

Note.

${ }^{a}$ Assuming half the IR flux and [C II] line luminosity of HZ10.

galaxies. The temperature $T$ is a primary parameter for characterizing the IR SED shape in our parameterization (see Section 2.1.1).

The total FIR luminosity at $z>5$ is usually obtained by integrating over an IR SED assuming priors on temperature and other parameters from the $z<4$ universe, which is then normalized to the $158 \mu \mathrm{m}$ continuum measurement of the galaxies. These parameters are typically $T=25-45 \mathrm{~K}$ $\left(T_{\text {peak }} \sim 18-28 \mathrm{~K}\right)$ and a range of $\alpha$ and $\beta_{\mathrm{IR}}$ corresponding to the average temperature of local (U)LIRGs (see C15). However, as shown in Section 2.3 by correlations in our local galaxy samples and analogues, there is evidence that this assumption might be incorrect for the average population of star-forming galaxies at $z>5$, which have lower metallicities and higher sSFRs. ${ }^{19}$

Figure 8 illustrates the change in total FIR luminosity $\left(\Delta \log \left(L_{\mathrm{FIR}}\right)\right)$ if the temperature $T$ or $T_{\text {peak }}$ is underestimated by the typical difference between average $z<4$ (U)LIRGs and the low-metallicity and high-sSFR analogues in our local samples. This difference can be up to $\sim 40 \mathrm{~K}(T=35 \mathrm{~K}$ instead of $T=75 \mathrm{~K})^{20}$, which would lead to a significant underestimation of $L_{\mathrm{FIR}}$ of $\sim 0.6 \mathrm{dex}$ as shown by the color gradient and black contours. The figure also shows that $T$ is the main parameter of uncertainty as $\Delta \log \left(L_{\text {FIR }}\right)$ only changes by \pm 0.04 dex over a large and conservatively estimated range of mid-IR slopes $\alpha$ and emissivities $\beta_{\mathrm{IR}}$.

The assumption of a warmer IR SED of high-redshift galaxies is supported by our study of local galaxies and lowredshift analogues, but also by several direct measurements in the literature.

\footnotetext{
19 Note that the constant background temperature emitted by the cosmic microwave background (CMB) is increased by a factor of 7 to $\sim 19 \mathrm{~K}$ at $z=6$. However, we expect this effect to increase the temperatures by less than $5 \mathrm{~K}$ on average at these redshifts (da Cunha et al. 2013), which is small given the uncertainties of our measurements.

${ }^{20}$ This corresponds to a difference between $T_{\text {peak }} \sim 23 \mathrm{~K}$ and $T_{\text {peak }} \sim 45 \mathrm{~K}$, i.e., $\Delta T_{\text {peak }} \sim 22 \mathrm{~K}$.
}

1. Álvarez-Márquez et al. (2016) stack FIR SEDs of $z \sim 3$ galaxies and find peak temperatures $T_{\text {peak }} \sim 30-40 \mathrm{~K}$ $(T \sim 45-65 \mathrm{~K})$, which are at the warm end of the distribution of temperatures of local galaxies.

2. Sklias et al. (2014) measure the temperatures of Herscheldetected star-forming galaxies at $1.5<z<3.0$ and find peak temperatures of $35 \mathrm{~K}<T_{\text {peak }}<50 \mathrm{~K}(55 \mathrm{~K}<$ $T<85 \mathrm{~K}$ ) in good agreement with our analogues. Also, Schreiber et al. (2017; see also Schreiber et al. 2015) and Knudsen et al. (2017; see also Watson et al. 2015) find temperatures of $T_{\text {peak }} \sim 40 \mathrm{~K}(T \sim 65 \mathrm{~K})$ for average galaxies at $z \sim 4$ and one galaxy at $z \sim 7.5$, respectively.

3. Strandet et al. (2016; see also Greve et al. 2012) present several strongly lensed galaxies at $z \sim 5-6$ that are detected and selected in the submillimeter by the South Pole Telescope (SPT) survey (Weiß et al. 2013) and observed between 100 and $3000 \mu \mathrm{m}$ by SPT, ALMA, APEX, Herschel, and Spitzer. We re-fit the FIR SEDs of these galaxies using the same method as our local samples and analogues and find temperatures of $60 \mathrm{~K}<T<80 \mathrm{~K}\left(37 \mathrm{~K}<T_{\text {peak }}<48 \mathrm{~K}\right)$, which is in good agreement with our local analogues (see Table 4 and black arrows in Figure 5).

4. The analysis of the starburst AzTEC-3 at $z=5.3$ in Riechers et al. (2014) also results in a temperature of $88_{-10}^{+10} \mathrm{~K}\left(T_{\text {peak }} \sim 51 \mathrm{~K}\right)$ and an SED similar to that of lowredshift analogues.

5. Finally, Pavesi et al. (2016) extended the FIR wavelength coverage of HZ6 and HZ10 from the C15 sample by measuring fluxes in the two sidebands at $158 \mu \mathrm{m}$ and $205 \mu \mathrm{m}$ independently with ALMA. Assuming a uniform prior for the temperature $\left(10-100 \mathrm{~K}\right.$, i.e., $5-60 \mathrm{~K}$ in $\left.T_{\text {peak }}\right)$ and $\beta_{\mathrm{IR}}=1.7 \pm 0.5$, they derive temperatures of $60_{-27}^{+35} \mathrm{~K}$ $\left(T_{\text {peak }} \sim 35 \mathrm{~K}\right)$ and $36_{-10}^{+25} \mathrm{~K}\left(T_{\text {peak }} \sim 25 \mathrm{~K}\right)$ for $\mathrm{HZ6}$ and HZ10, respectively. As the authors discuss, these temperature measurements are very uncertain as the current data do not cover wavelengths near the IR peak that are sensitive to warm dust. An additional measurement at bluer 
wavelengths (e.g., rest-frame $122 \mu \mathrm{m}$; see Appendix) would increase the confidence in the estimated temperature.

Following the above reasoning, we recompute the total FIR luminosities of the $12 z \sim 5.5$ systems from $\mathrm{C} 15$ assuming a temperature prior of $60 \mathrm{~K}<T<90 \mathrm{~K}\left(T_{\text {peak }} \sim 35-50 \mathrm{~K}\right)$ for the IR SED. As in C15, we create IR SEDs using the Casey (2012) parameterization that we normalize to the observed $158 \mu \mathrm{m}$ continuum emission of the galaxies, which is the only data point available in the IR. The result is marginalized over a grid of mid-IR slopes and emissivities $(1.5<\alpha<2.5$, $\left.1.0<\beta_{\mathrm{IR}}<2.0\right)$. The total FIR luminosity is derived by integration between 3 and $1100 \mu \mathrm{m}$, and the errors are derived from the uncertainty in the photometric measurement at $158 \mu \mathrm{m}$ and the marginalization over the assumed range in $\alpha$ and $\beta_{\mathrm{IR}}$. We list the updated $L_{\mathrm{FIR}}$ values in Table 4 , which, as expected, are increased by $\sim 0.6$ dex compared to the previous results assuming a temperature prior with $25 \mathrm{~K}<T<45 \mathrm{~K}$ $\left(T_{\text {peak }} \sim 18-28 \mathrm{~K}\right)$ as in $\mathrm{C} 15$.

\subsection{Constraints on Metallicity and sSFR as Derived from Spitzer Colors}

The strong emission lines in high-redshift galaxies can alter their observed photometry, which in turn allows us to study them. In particular, Spitzer observations allow us to constrain the fluxes of strong optical lines such as [O III] and $\mathrm{H} \alpha$ of galaxies at $z>3$, and thus can put strong constraints on their (specific) SFR and metal content, and hence the evolutionary stage of these galaxies (e.g., Shim et al. 2011; Faisst et al. 2016a; Rasappu et al. 2016).

Figure 9 shows the expected $[3.6 \mu \mathrm{m}]-[4.5 \mu \mathrm{m}]$ and $[4.5 \mu \mathrm{m}]-[5.8 \mu \mathrm{m}]$ colors in the redshift range $5.1<z<5.8$ for different $\mathrm{H} \alpha$ EWs and $[\mathrm{O} \mathrm{III]} / \mathrm{H} \alpha(\propto[\mathrm{O} \mathrm{III}] / \mathrm{H} \beta)$ emission line ratios. ${ }^{21}$ The black symbols show the dust-corrected ${ }^{22}$ colors of the galaxies in the C15 sample with their names indicated. Note that while all galaxies have reliable measurements at 3.6 and $4.5 \mu \mathrm{m}$, only three galaxies have measured $5.8 \mu \mathrm{m}$ fluxes due to the only shallow coverage at $5.8 \mu \mathrm{m}$. Furthermore, the latter measurements have large uncertainties and are therefore are only meaningful for HZ1 and HZ6. For galaxies at $5.1<z<5.3$, the $[3.6 \mu \mathrm{m}]-[4.5 \mu \mathrm{m}]$ color is a good estimator of the $\mathrm{H} \alpha$ equivalent width (e.g., Faisst et al. 2016a; Rasappu et al. 2016), $\mathrm{EW}(\mathrm{H} \alpha)$, while at higher redshifts it becomes degenerate with the $[\mathrm{O} \mathrm{III}] / \mathrm{H} \alpha$ ratio. Both Spitzer $[3.6 \mu \mathrm{m}]-[4.5 \mu \mathrm{m}]$ and $[4.5 \mu \mathrm{m}]-[5.8 \mu \mathrm{m}]$ colors suggest $\mathrm{EW}(\mathrm{H} \alpha)>500 \AA$ integrated over the three subcomponents of HZ6, which could indicate a recent starburst event that might be induced by the tidal interaction between these three galaxies. In comparison, these colors suggest $\operatorname{EW}(\mathrm{H} \alpha)<500 \AA$ for $\mathrm{HZ1}, \mathrm{HZ7}$, and HZ8. In addition, we estimate [O III] $/ \mathrm{H} \alpha \lesssim 1$ (or $[\mathrm{O} \mathrm{III}] / \mathrm{H} \beta<2.86$ ) for $\mathrm{HZ} 9$ and $\mathrm{HZ10}$ and $[\mathrm{O} \mathrm{III}] / \mathrm{H} \alpha \gtrsim 1$ (or $[\mathrm{O} \mathrm{III}] / \mathrm{H} \beta>2.86$ ) for $\mathrm{HZ1}, \mathrm{HZ2}$, and $\mathrm{HZ} 4$ from their [3.6 $\mu \mathrm{m}]-[4.5 \mu \mathrm{m}]$ colors. Assuming all of these galaxies sit on the BPT "main-sequence" locus of high-redshift galaxies (e.g., Kewley et al. 2013; Steidel et al. 2014; Masters et al. 2016; Sanders et al. 2016; Strom et al. 2017), we can relate the

\footnotetext{
$\overline{21}$ We assume templates from the Bruzual \& Charlot (2003) library with halfsolar (stellar) metallicity and a $500 \mathrm{Myr}$ old constant SFH. As shown in Faisst et al. (2016a), the choice of these parameters, as long as it is reasonable, has little impact on the intrinsic model color $(<0.15$ mag for $z>5)$.

${ }^{22}$ For dust correction, we apply the maximum of the $E(B-V)$ calculated directly from $L_{\mathrm{FIR}} / L_{\mathrm{UV}}$ using the relation between IRX and $A_{1600}$ (Meurer et al. 1999) and from $\beta$ using the relation between $A_{1600}$ and $\beta$ (Calzetti et al. 2000).
}

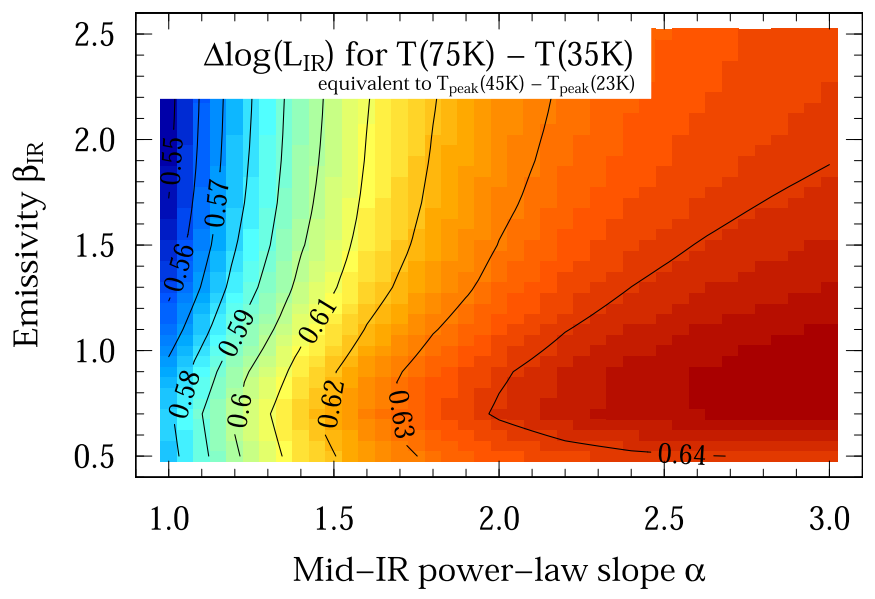

Figure 8. Impact of a "warm" IR SED on the total FIR luminosity measurements in the Casey (2012) parameterization. The color and contours show the increase in $\log L_{\mathrm{FIR}}$ for a change in luminosity-weighted temperature from $T=35 \mathrm{~K}$ to $T=75 \mathrm{~K}$ (corresponding to a change in $T_{\text {peak }}$ from $23 \mathrm{~K}$ to $45 \mathrm{~K})$ as a function of the mid-IR slope $\alpha$ and the emissivity $\beta_{\mathrm{IR}}$. The total luminosity increases by $0.56-0.64 \mathrm{dex}$ across the range in $\alpha$ and $\beta_{\mathrm{IR}}$ for $\Delta T=40 \mathrm{~K}$. Therefore, $T$ is the main parameter that defines $L_{\mathrm{FIR}}$, and its measurement is important to obtain accurate $L_{\mathrm{FIR}}$ measurements in highredshift galaxies where only a few data points are available in the FIR.

[O III] $/ \mathrm{H} \alpha$ line ratio to $[\mathrm{N} \mathrm{II}] / \mathrm{H} \alpha$, which is a proxy for the gasphase metallicity. Hence, we would expect $[\mathrm{N} \mathrm{II}] / \mathrm{H} \alpha>-0.6$ for HZ9 and HZ10, which translates into a gas-phase metallicity of $12+\log (\mathrm{O} / \mathrm{H}) \gtrsim 8.5$ according to Curti et al. (2017). The same reasoning leads to $12+\log (\mathrm{O} / \mathrm{H}) \lesssim 8.5$ for $\mathrm{HZ1}, \mathrm{HZ2}$, and HZ4. Note that these metallicity estimates are in good agreement with the strong UV absorption features seen in these galaxies (Barišić et al. 2017).

Overall, this suggests that low-metallicity, high-sSFR, lowredshift galaxies are indeed good analogues for high-redshift systems and that we should be adopting higher temperatures for the FIR SEDs. Interestingly, the exception is HZ10, which red Spitzer colors and rest-UV spectrum (Section 4.2) indicate a more mature system with higher metallicity and lower sSFR in line with the cooler temperature estimated by Pavesi et al. (2016) and being the strongest FIR continuum emitter in the C15 sample.

\subsection{Comparison of UV, FIR, and [C II] Morphologies}

The galaxies in C15 are all resolved in the ALMA FIR line and continuum observations at a resolution of 0 ". 5 , which allows, together with their high-resolution near-IR HST images, a spatial study of rest-frame UV (a proxy for unobscured star formation) and FIR continuum (a proxy for the dust distribution and obscured star formation) in typical $z>5$ galaxies for the first time (Figure 10).

The FIR continuum-detected galaxy HZ9 shows no offset between UV and FIR emission, indicative of a dust-obscured compact central star formation region. In contrast, the FIR emission of HZ4 and HZ10 is offset by up to $5 \mathrm{kpc}$ from the location of the unobscured star formation, which could indicate a substantial redistribution of dust clouds due to UV radiation pressure, tidal interactions with a recent or ongoing merger event, or the production and growth of dust caused by a recent starburst (c.f. Schneider et al. 2016). Such an offset of FIR continuum and unobscured star formation is also seen in the two local analogues (GALEX 1000+0157, GALEX 1000 

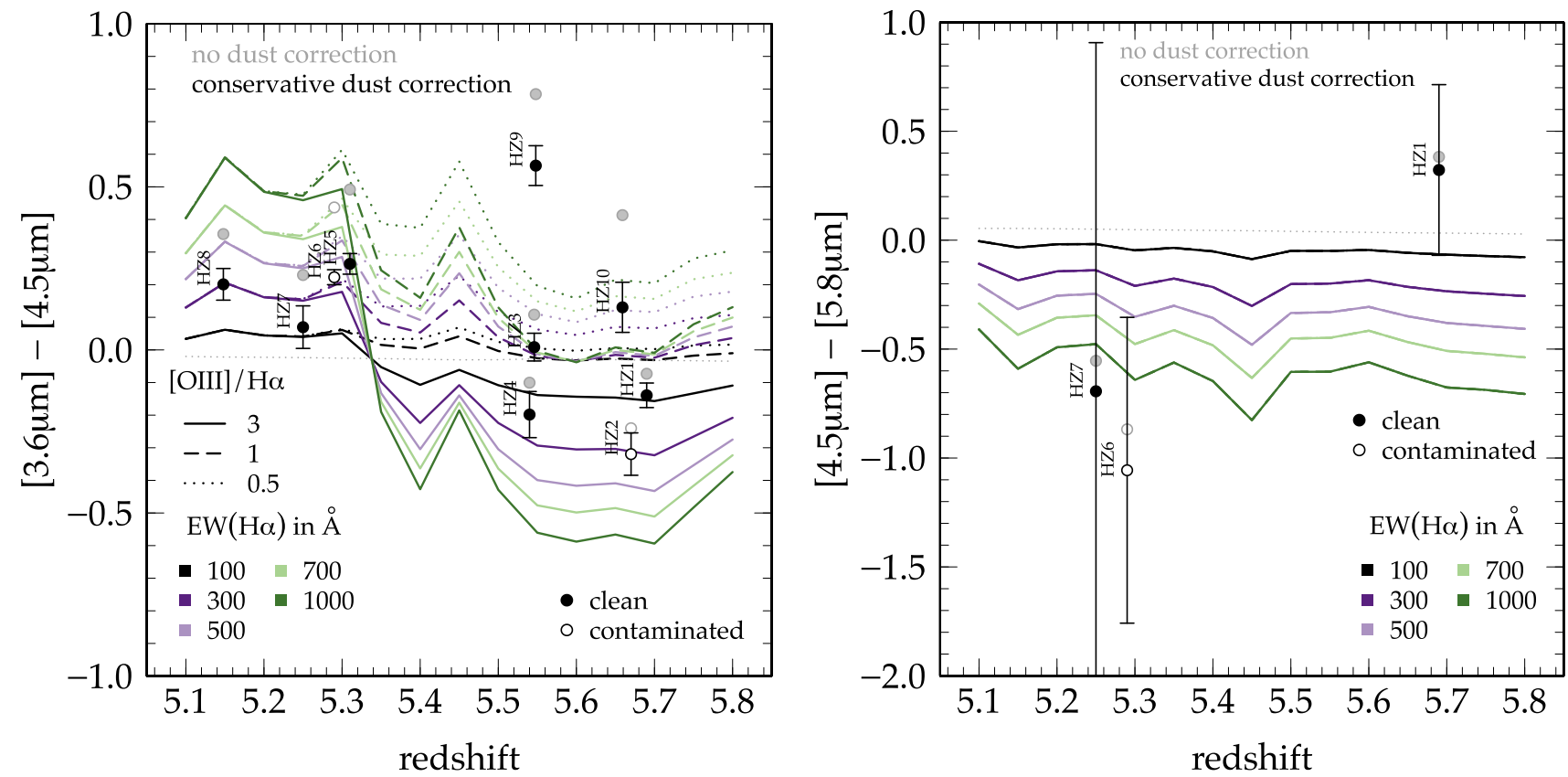

Figure 9. Estimation of $\mathrm{EW}(\mathrm{H} \alpha)$ and $[\mathrm{O} \mathrm{III}] / \mathrm{H} \beta$ line ratios from Spitzer/IRAC photometry for the $z \sim 5.5$ galaxies. The two panels show Spitzer [3.6 $\mu \mathrm{m}]-[4.5 \mu \mathrm{m}]$ (left) and $[4.5 \mu \mathrm{m}]-[5.8 \mu \mathrm{m}]$ (right) for a suite of models with different $\mathrm{EW}(\mathrm{H} \alpha)$ and $[\mathrm{O} \mathrm{III}] / \mathrm{H} \alpha$ ratios (see the text). The black points show the data for the $z \sim 5.5$ galaxies corrected for dust (using the maximal $E(B-V)$ derived from either IRX or $\beta$; see text), and the gray points show the observed data (no dust correction). We indicate galaxies with contaminated Spitzer photometry, e.g., due to multiple components (empty points).

$+0201)$ with the lowest metallicities and lowest dust content (i.e., IRX) as shown in the upper panels of Figure 10.

Emission from singly ionized carbon is commonly originating from the photodissociation regions (PDRs) on the surfaces of molecular clouds. The [C II] luminosity $\left(L_{[\mathrm{C} \mathrm{II}]}\right)$ is therefore used as a proxy for the star formation in galaxies; therefore, it should coincide well with the locations of star formation (e.g., Carilli \& Walter 2013; De Looze et al. 2014). The sample of $\mathrm{C} 15$, however, shows offsets between [C II] and UV emission of up to $5 \mathrm{kpc}$ as well as a very extended [C II] emission over 5-10 kpc, a factor of $\sim 5$ larger than the typical UV size of the galaxies (Figure 10). The extended [C II] emission could indicate that a non-negligible fraction of [C II] is originating from the warm neutral gas in the diffuse ISM that is heated by the strong UV radiation of young massive stars in high-redshift galaxies (e.g., Pineda et al. 2013; Díaz-Santos et al. 2014; Herrera-Camus et al. 2015; Vallini et al. 2015). Furthermore, recent hydrodynamical simulations suggest that offsets of up to $10 \mathrm{kpc}$ between the [C II] emission and regions of ongoing star formation are expected in young primeval galaxies at high redshift due to their strong stellar radiation pressure (Vallini et al. 2013; Maiolino et al. 2015).

Overall, the FIR, [C II], and UV morphologies would support a picture of a turbulent, low dust-column density ISM with a greater optically thin fraction, which would cause a higher observed temperature. This is consistent with our analysis of the low metallicities and high sSFRs of local galaxies (Section 2.3) and could suggest a change in the relation between SFR and $L_{[\mathrm{C} \text { II] }}$ found in galaxies at lower redshifts (e.g., Aravena et al. 2016).

Alternatively, the offsets described above could be due to differential dust obscuration as is commonly observed in SMGs and DSFGs (Riechers et al. 2014; Hodge et al. 2015, 2016). For example, [C II] is much less affected by dust obscuration and can therefore be detected at places where the UV light is completely absorbed, i.e., places of obscured star formation. On the other hand, the [C II] must then be destroyed or expelled at locations of unobscured star formation.

\subsection{Updated IRX $-\beta$ Diagram of $\mathrm{z} \sim 5.5$ Galaxies}

Figure 11 shows the IRX $-\beta$ diagram at $z \sim 5.5$ using the $H S T$-based $\beta$ measurements (Barišić et al. 2017) and $L_{\mathrm{FIR}}$ derived for a maximally warm IR SED prior $(60 \mathrm{~K}<T<90 \mathrm{~K}$, $\left.T_{\text {peak }} \sim 35-50 \mathrm{~K}\right)$ for the galaxies in C15. The FIR continuumdetected high-redshift galaxies are shown as solid red symbols, while the non-detections are shown as empty red symbols with arrows. FIR-detected companion galaxies are shown as empty red symbols. Previous measurements (if available) that assume a cooler IR SED prior of $T=25-45 \mathrm{~K}\left(T_{\text {peak }} \sim 18-28 \mathrm{~K}\right)$ are shown as light red points.

If the warmer IR SED priors are correct, this would push the galaxies up by $\sim 0.6$ dex in IRX. This brings the galaxies at low IRX in better agreement with known dust properties measured in local starbursts (Meurer et al. 1999; and the re-examination by Takeuchi et al. 2012) and models adopted for the metal-poor SMC (Pettini et al. 1998; Reddy et al. 2006). We note that the applied warm IR SED prior is motivated by the extrapolation of trends found in local galaxy samples as well as in the three lowredshift analogues, which we think are reasonable representatives of a large fraction of high-redshift galaxies. However, the true temperature distribution of typical high-redshift galaxies is still unknown, and we therefore argue that our temperature prior is a very reasonable upper limit and so are the updated IRX values. Taking the early measurements by Pavesi et al. (2016) as well as the trends in the local sample at face value, a temperature gradient across the IRX- $\beta$ diagram is not unexpected. For example, lower temperatures for HZ9 and HZ10, which would reduce their IRX and bring them in better agreement with the local starburst and the Takeuchi et al. 

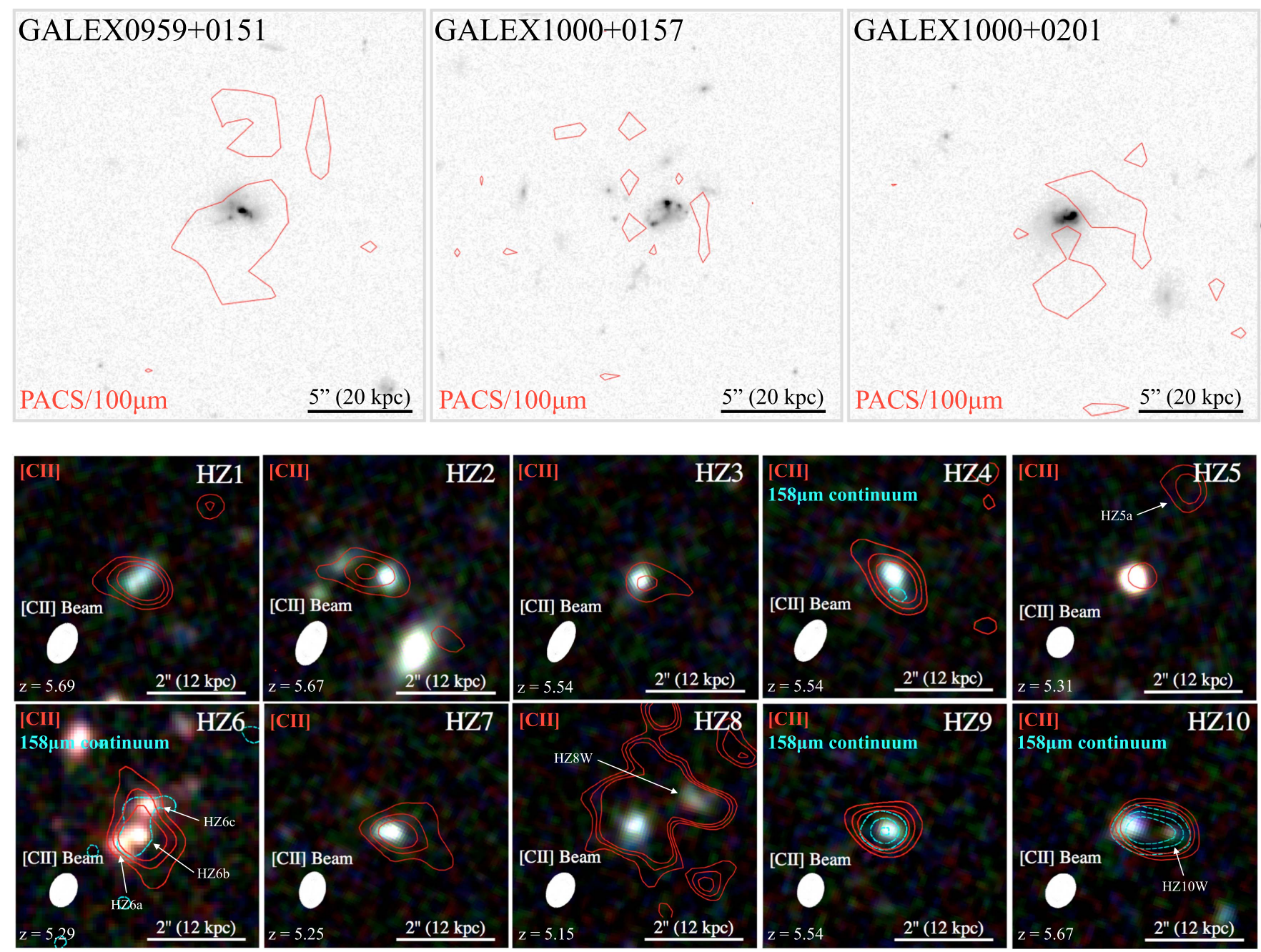

Figure 10. Spatial location of the UV and FIR emission of the three analogues (upper large panels) and $z \sim 5.5$ galaxies from C15 (lower small panels). For the analogues, we show the ACS/F814W (gray scale) together with the PACS $/ 110 \mu \mathrm{m}$ emission (red contours) for $2 \sigma, 6 \sigma$, and $10 \sigma$. For the $z \sim 5.5$ galaxies, we show the F105W $+\mathrm{F} 125 \mathrm{~W}+\mathrm{F} 160 \mathrm{~W}$ color cutouts together with the FIR [C II] line emission (red contours) and the continuum at $158 \mu \mathrm{m}$ (cyan dashed contours), both shown for $2 \sigma, 6 \sigma$, and $10 \sigma$. The [C II] beam FWHM is indicated by the white ellipse. Note the offset in [C II] from the UV emission (indicating regions of unobscured star formation) and the extended [C II] emission, potentially indicating a turbulent and warm ISM due to the strong UV radiation of young stars.

(2012) relation, are entirely possible. More direct measurements of the IR SED at high redshifts and larger samples of low-redshift analogues are necessary to study such effects.

We find that galaxies at $z \sim 5.5$ occupy a large fraction of the IRX $-\beta$ diagram with significant scatter, which indicates a large variety in their physical properties already 1 billion years after the big bang. This scatter has to be understood in order to use the IRX $-\beta$ relation to predict the dust properties of highredshift galaxies from UV colors only, as is commonly done. The current sample of galaxies is too small to make such statistical predictions as well as point out the precise trends on the IRX $-\beta$ diagram as a function of galaxy properties. In the following, we can therefore only derive a patchy understanding of how galaxies behave in relation to $\beta$ and IRX.

We find galaxies with blue UV colors $(\beta<-1.5)$ and large IRX values on or above the curve for local starbursts (HZ9, HZ10). As discussed in Section 4.2, these are likely among the most metal-rich and mature main-sequence galaxies at these redshifts. The blue UV color of these galaxies could be explained by a recent perturbation of the dust clouds by tidal interactions or a patchy optically thick dust screen
(Section 3.2). The former could be the case for HZ10 showing a large offset between the UV and FIR emission, while the latter might be the case for HZ9, for which the UV and FIR emission coincide (Section 4.3). We note that HZ4, which is also elevated somewhat above the location of local galaxies but not as much as the others, is expected to be less metal enriched. Also, its morphology (Figure 10) shows that its FIR emission is offset in a tail pointing to the northeast. The large fraction of unobscured UV emission along the line of sight causes its blue UV color $(\beta \lesssim-2)$, but its substantial dust mass causes a high IRX value.

Next to galaxies showing increased IRX values, we find galaxies with low IRX values (even upper limits) and red UV colors (e.g., HZ6a, HZ7, HZ8, or HZ8W). This is even the case for a maximally warm IR SED prior that is assumed here and would be even more significant if no temperature evolution is assumed. Such galaxies are expected to have a significant lineof-sight dust attenuation but only little dust FIR emission, i.e., a low total dust mass. The recent $H S T$ observations provide very accurate $\beta$ measurements of these galaxies as shown by the simulations in Barišić et al. (2017). Therefore, we conclude that 


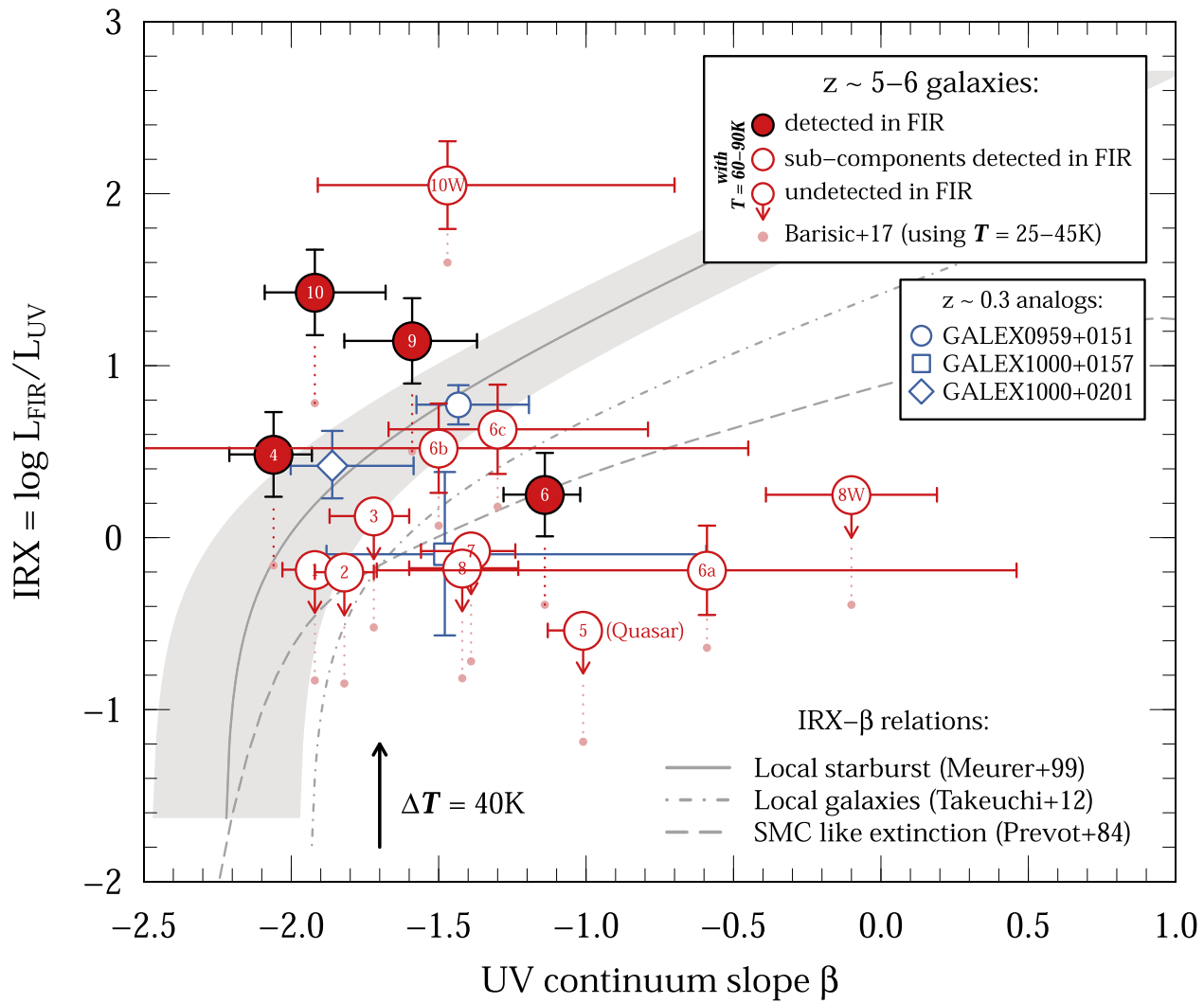

Figure 11. IRX $-\beta$ diagram at $z \sim 5.5$ for a maximally warm IR SED prior with luminosity-weighted temperature $T=60-90 K\left(T_{\text {peak }} \sim 35-50 \mathrm{~K}\right)$. We show the $z=5-6$ galaxies from Barišić et al. (2017) with FIR detection (red filled circles) and without FIR detection (empty circles, upper limits), as well as the FIR-detected subcomponents as described in their paper (empty circles). The position of the galaxies with a cooler IR SED prior $\left(T=25-45 \mathrm{~K}\right.$, i.e., $\left.T_{\text {peak }} \sim 18-28 \mathrm{~K}\right)$ as assumed previously (e.g., Capak et al. 2015) is indicated by faint red dots. The arrow indicates the change in IRX for $\Delta T=40 \mathrm{~K}$. Our three $z \sim 0.3$ analogues is shown in blue and are consistent with the location of the high-redshift galaxies. Common IRX - $\beta$ relations for local starbursts (Meurer et al. 1999, with scatter; Takeuchi et al. 2012; and galaxies with SMC-like dust, Prevot et al. 1984; Pettini et al. 1998) are indicated by solid, dotted-dashed, and long-dashed gray lines, respectively. Note that the upper limits of the FIR-undetected $z=5-6$ galaxies are mostly consistent with the IRX $-\beta$ relation expected for SMC-like dust using the maximally warm IR SED prior. However, some galaxies still show a deficit in IRX compared to the SMC relation and cannot be explained by common analytical models of dust attenuations in galaxies (see Section 4.5).

these galaxies have a deficit in IRX and are not biased in $\beta$. Also, the UV spectra and Spitzer colors strongly suggest that these galaxies are young and not dominated by old stellar populations that could cause a red UV color (e.g., Howell et al. 2010; Narayanan et al. 2017; Popping et al. 2017a). These galaxies are curious because such a configuration of IRX and $\beta$ is difficult to explain with current models of dust attenuation in galaxies. As shown in Section 3.1, the simple analytical model of Charlot \& Fall (2000) cannot cover that part of the parameter space even assuming low dust opacities and a steep SMC-like dust attenuation curve. From the Spitzer colors (Section 4.2) as well as UV spectra (Barišić et al. 2017), we would expect these galaxies to be primeval and metal- poor. The large offsets between [C II] emission and star-forming regions, and the extent of the [C II] emission could be indicative of vigorous star formation, strong stellar radiation pressure, and substantial heating of the diffuse ISM. We suggest that the enhanced turbulence in these galaxies paired with merger events significantly alters their spatial dust distribution. In the next section, we propose an updated model for dust absorption in the ISM of these galaxies that can explain the location of these peculiar galaxies on the IRX $-\beta$ diagram by taking into account geometrical effects of the dust and star distribution.

\subsection{Dust Model for FIR-faint High-z Galaxies: Separating Clouds from Stars}

As discussed in Section 3.1, a simple analytical model for dust absorption in galaxies with well-mixed dust and stars in thermal equilibrium (Charlot \& Fall 2000) is able to explain the majority of galaxies on the IRX $-\beta$ diagram. However, it is not able to explain the FIR-faint galaxies at very low IRX at a given UV color found at $z>5$ even for low dust opacities and a steep SMC-like dust attenuation curve. In order to explain the location of these galaxies, the dust clouds have to be in a configuration that allows them to attenuate the UV light enough to cause the red $\beta$, but at the same time, the FIR emission has to be decreased by $0.5-1.0 \mathrm{dex}$ with respect to SMC-like galaxies.

We propose that this can be achieved by a spatial separation of the clouds and the star-forming regions that naturally occurs as the birth clouds are disrupted by the radiation pressure of the stars and turbulences in the ISM. Instead of letting the birth clouds disappear after $10 \mathrm{Myr}$ (the typical lifetime of $\mathrm{O}$ and $\mathrm{B}$ stars), we modify the Charlot \& Fall (2000) prescription such that the birth clouds are separated from the star-forming regions by $\delta s(t)=t \times \delta v$ with $t<10 \mathrm{Myr}$. The total FIR luminosity is decreased roughly by a factor of $\propto 1 / \delta s^{2}$ (where $\delta s$ is the distance between the star-forming region and cloud) due to the 


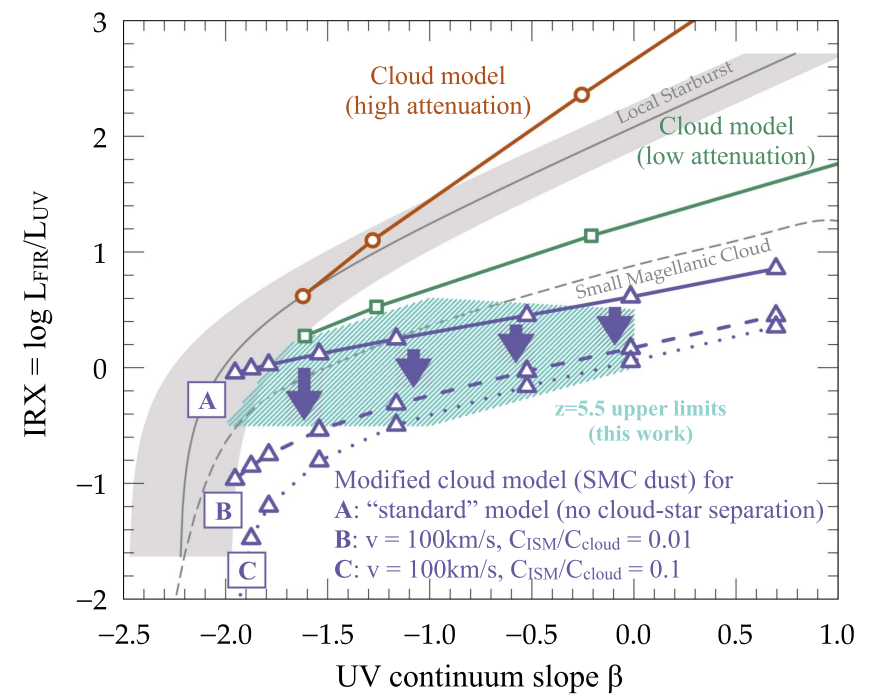

Figure 12. Same as Figure 6, but here including our proposed updated model that can explain the low IRX values of the FIR-undetected $z>5$ galaxies (cyan hatched region). The revised model takes into account the separation of clouds and young stars due to the increased turbulent motion in high-redshift galaxies. Shown are two different cloud column density contrasts of 0.01 (long-dashed line with blue triangles) and 0.1 (dotted line with blue triangles), and a turbulent motion of $v \approx \sigma_{\text {ISM }} \approx 100 \mathrm{~km} \mathrm{~s}^{-1}$ of the surrounding warm ISM is assumed.

smaller angle along the line of sight covered by the cloud, while the UV color from the obscured regions is not significantly changed.

We assume that initially, the star clusters have the same velocity as the birth cloud. After that, the cloud is subject to hydrodynamic forces and pressures, e.g., caused by the radiation of young stars or merging events, which causes the clouds to run into the nearby low-density warm ISM. The velocity change of the clouds can be estimated by a momentum conservation argument and depends on the column density ratio of the clouds and the surrounding ISM as well as the relative velocity $v$ of the external medium,

$$
\delta v \approx\left(\frac{C_{\mathrm{ISM}}}{C_{\text {cloud }}}\right) \times v .
$$

The turbulent velocity dispersion of the ISM $\left(\sigma_{\text {ISM }}\right)$ provides a reasonable estimate for $v$. From the [C II] emission line widths of the $z \sim 5.5$ galaxies measured by $\mathrm{C} 15$, we find $v$ $\sim 100 \mathrm{~km} \mathrm{~s}^{-1}$ and we take this value to approximate $\sigma_{\text {ISM }}$ in the following. Note that a significant part of the [C II] line width may be due to rotation as suggested for HZ9 and HZ10 (G. Jones et al. 2017, in preparation). Therefore, this approximation might lead to an upper limit for $\sigma_{\text {ISM }}$. Our simple modified model is shown in Figure 12 by the dashed and dotted blue lines for $C_{\mathrm{ISM}} / C_{\text {cloud }}$ of 0.01 and 0.1 , respectively, assuming an SMC-like wavelength dependence of the optical depth of the ambient ISM.

Our model can quantitatively explain the low IRX values of the $z \sim 5.5$ galaxies and can also naturally lead to the large observed range in $\beta$ at a fixed IRX value. Depending on the geometry and viewing angle of the observer, the dust clouds might be spatially offset from the line of sight, and the UV light can escape the galaxy without being attenuated, which would cause blue UV colors but very similar IRX values.

Qualitatively, this model is also consistent with other observed characteristics of high-redshift galaxies such as increased turbulent motion, increased radiation pressure, merger events, and strong gas inflows. In all cases, this leads to dust that is not well mixed with the stars, with isolated ISM clouds spatially offset from the regions of star formation. At the same time, low opacity dust screens due to tidal disruption or from the diffuse ISM can be created in front of the stars, producing the observed red UV colors. This set-up could create lines of -sight with attenuated UV light of the star-forming regions as well as ones that are clear, at a fixed IRX. In fact, we see hints of such behavior in local galaxies. NGC 5253, NGC 1568, and Zwicky 403 are examples of local galaxies that have similar morphological properties, although the $z>5$ population is likely more extreme (c.f. Gil de Paz et al. 2007). All three galaxies show confined isolated clouds of gas and dust along with clear lines of sight to the star-forming regions with a complex morphology.

\subsection{Other Possible Geometric Configurations}

There are other geometric configurations that can cause the observed low IRX values and red UV spectral slopes. Radiation pressure and tidal forces can stir up the ISM in these young primeval galaxies and could result in a large fraction of dust and gas that is expelled into their circumgalactic regions. The low-luminosity quasar HZ5 has evidence suggesting an extended halo of dust and gas out to several kiloparsecs around its [C II] confirmed (foreground) companion HZ5a. The spectrum of the quasar shows absorption features at the [C II] redshift of HZ5a as well as a narrow Ly $\alpha$ emission line possibly caused by absorption in the foreground galaxy (Capak et al. 2011). Such circumgalactic dust can attenuate the UV light of a companion galaxy in the background and cause a red $\beta$. At the same time, its IRX value is determined by the foreground galaxy's dust. The galaxy HZ8W (a [C II]confirmed companion of HZ8) could be an example of such a geometrical configuration. It is characterized by a red spectral slope of -0.1 and an upper limit in IRX of only 0.2 . The UV light of this galaxy could be substantially attenuated by a foreground screen of dust and gas originating from the larger and more massive galaxy HZ8 at several kiloparsecs projected distance. This is also indicated by its very extended [C II] emission. The HZ6 system could show a similar configuration.

\subsection{Correlation with Stellar Mass}

Several studies indicate a relation between IRX and stellar mass for galaxies at $z<4$ (e.g., Pannella et al. 2009; Heinis et al. 2014; Pannella et al. 2015; Álvarez-Márquez et al. 2016). The growing samples at $z>4$ show that this relation might break down at higher redshifts (e.g., Fudamoto et al. 2017). Specifically, it is found that these galaxies show a lower IRX at a fixed stellar mass than expected from the relations at lower redshifts. Part of the reason for this discrepancy is the unknown IR SED of the galaxies (Bouwens et al. 2016; Fudamoto et al. 2017). Figure 13 shows the IRX versus stellar mass diagram for the $z=5-6$ galaxies assuming a warmer IR SED prior of $T=60-90 \mathrm{~K}\left(T_{\text {peak }} \sim 35-50 \mathrm{~K}\right)$ together with data and relations at lower redshift taken from the literature. Even with a warmer IR SED, the FIR-undetected galaxies still fall below the expected IRX versus stellar mass relation found at $z<4$. Assuming that the stellar masses are robust, this suggests that the stellar mass becomes uncorrelated with IRX (i.e., total dust) at $z>5$, similar to the relation between IRX and $\beta$. This could 


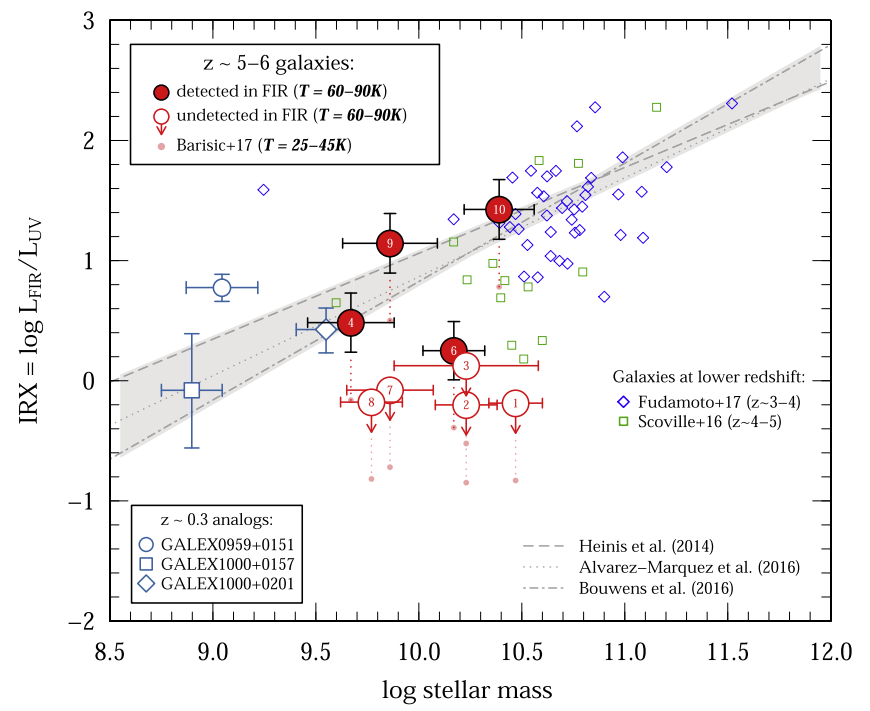

Figure 13. IRX vs. stellar mass diagram for galaxies at $z=5-6$ assuming a maximally warm IR SED (large red symbols) compared to galaxies at $z=3-4$ (blue diamonds; Fudamoto et al. 2017), $z=4-5$ (green squares; Scoville et al. 2016), and our three analogues (large blue symbols). Relations derived at $z \sim 2$ -4 are shown in gray (dashed: Heinis et al. 2014; dotted: Álvarez-Márquez et al. 2016; dotted-dashed: Bouwens et al. 2016).

be due to mergers, which would increase the stellar mass without necessarily changing the IRX, or a modified geometric distribution of dust as discussed here. Alternatively, it could be that at these redshifts the production of dust is lagging behind the mass growth of the galaxies, which happens on very short timescales.

As mentioned in Section 4.4, such defined relations between IRX and $\beta$ as well as stellar mass are important to predict the dust properties of large samples of high-redshift galaxies from quantities measured in the rest-frame UV and optical. Unfortunately, we currently lack large enough galaxy samples to define such relations accurately. Large ALMA programs targeting the FIR of high-redshift galaxies will be crucial in the future to progress.

\section{Summary and Conclusion}

Observations of the IRX $-\beta$ relation across cosmic time is important for understanding the evolution of the ISM and dust properties of galaxies in the early universe. Recent studies of the IRX $-\beta$ relation of main-sequence galaxies at $z>3$ have reported a significant evolution of this relation. Because the UV colors are accurately measured by $H S T$, this evolution is attributed to a deficit in IRX at fixed $\beta$ compared to galaxy samples at lower redshifts with similar stellar masses. These low IRX values and large ranges in UV color are curious, since they cannot be explained by standard models of dust attenuation in galaxies. The total FIR luminosity of these high-redshift samples is very poorly constrained often with only one data point at these wavelengths. A common solution to this discrepancy is therefore an evolving temperature, which is the most important parameter to define the IR SED. On the other hand, a different geometric distribution of dust and stars can be responsible for the evolution of the IRX $-\beta$ relation to high redshifts. In this work, we investigated both ideas, and also find that with a "maximally" warm IR SED, the distribution of dust and stars likely needs to be altered to explain the location of some of the $z>5$ galaxies on the IRX $-\beta$ plane.

We first infer the IR SED shape of high-redshift galaxies by using correlations of the luminosity-weighted temperature (in this work referred to as "temperature") with the metallicity and sSFR in samples of nearby galaxies, which we extend with the extreme cases of three $z \sim 0.3$ analogues of $z>5$ galaxies. This provides us with good evidence that the IR SEDs of high-redshift galaxies show a warm emission component, as suggested by temperatures of up to $T=60-90 \mathrm{~K}\left(T_{\text {peak }} \sim 35-50 \mathrm{~K}\right)$, which is significantly warmer than that of typical $z<3$ galaxies $(T \sim 25-45 \mathrm{~K}$, $T_{\text {peak }} \sim 18-28 \mathrm{~K}$ ). We believe that the warmer IR SEDs are due to a combination of lower ISM optical depth, and denser and stronger UV radiation fields associated with more intense star formation and low-metallicity environment. A comparison of the UV, [C II], and FIR morphologies along with the metallicities in high- and low-redshift systems may support this picture.

Assuming such maximally warm IR SEDs would increase the FIR luminosity (and therefore IRX) by up to 0.6 dex (factor of 4) with respect to previous measurements that assume the cooler, on average, IR SEDs of local galaxies, this reduces the deficit in IRX and brings most of the galaxies at $z>5$ in agreement with the relation measured at lower and local redshifts, albeit with significant scatter. Even if the assumption of such warm IR SEDs is correct, a population of galaxies with red UV colors and low IRX remains. We suggest that the geometric distribution of dust and stars is altered in highredshift galaxies (see summarizing schematic in Figure 14).

Common dust attenuation models assuming a well-mixed ISM (Charlot \& Fall 2000) are not able to explain the galaxies with low IRX values and a large range in UV colors. The large spatial offsets between star-forming regions and [C II] emission as well as the Spitzer colors suggest that these galaxies are young, metal poor, and likely have enhanced cloud motions due to stellar radiation pressure. Based on this, we propose an updated dust attenuation model in which the dust clouds are separated from young stars by their radiation pressure. We show that such a separation can be easily achieved within the lifetime of massive $\mathrm{O}$ and $\mathrm{B}$ stars in young star-forming galaxies at high redshifts. Such a model can successfully reproduce the location of FIR-faint galaxies with low IRX. Furthermore, it can naturally reproduce the large scatter in UV colors due to viewing-angle effects.

We note that the evidence of a warmer IR SED in highredshift galaxies is indirectly derived from correlations in local galaxies and three exemplary analogues of $z>5$ galaxies and the fact that high-redshift galaxies are metal poor and have high sSFRs. However, we stress that a direct measurement of, e.g., the temperature $T$ is absolutely crucial to verify these trends. Unfortunately, such measurements turn out to be time consuming and only possible for some of the brightest highredshift galaxies we know to date (see Appendix). The first estimates of the temperature of two normal $z \sim 5.5$ galaxies suggest large variations, highlighting the importance of such direct measurements (Pavesi et al. 2016). The two galaxies occupy two different regions on the IRX $-\beta$ diagram and likely are different in terms of metallicity and sSFR despite having comparable UV emission. This hints toward a large diversity in the galaxy population at $z>5$, only 1 billion years after the big bang, that is waiting to be explored with further observations. 
The authors would like to thank Nick Scoville, Caitlin Casey, Ranga-Ram Chary, Rychard Bouwens, Kirsten Larson, Shoubaneh Hemmati, and Lee Armus for valuable discussions which improved this manuscript. Furthermore, the authors thank the anonymous referee for the valuable feedback. D.R. and R.P. acknowledge support from the National Science Foundation under grant number AST-1614213 to Cornell University. R.P. acknowledges support through award SOSPA3-008 from the NRAO. We thank the ALMA staff for facilitating the observations and aiding in the calibration and reduction process. This paper makes use of the following ALMA data: ADS/JAO.ALMA\#2011.0.00064.S, ADS/JAO. ALMA\#2012.1.00523.S, ADS/JAO.ALMA\#2012.1.00919. $\mathrm{S}$, and ADS/JAO.ALMA\#2015.1.00928.S. ALMA is a partnership of ESO (representing its member states), NSF (USA), and NINS (Japan), together with NRC (Canada), NSC and ASIAA (Taiwan), and KASI (Republic of Korea), in cooperation with the Republic of Chile. The Joint ALMA Observatory is operated by ESO, AUI/NRAO, and NAOJ. This work is based in part on observations made with the Spitzer Space Telescope and the W.M. Keck Observatory, along with archival data from the NASA/ESA Hubble Space Telescope, the Subaru Telescope, the Canada-France-Hawaii Telescope, and the ESO Vista telescope obtained from the NASA/IPAC Infrared Science Archive. This research made use of the NASA/IPAC Extragalactic Database (NED), which is operated by the Jet Propulsion Laboratory, California Institute of Technology, under contract with the National Aeronautics and Space Administration.

\section{Appendix \\ Quantifying the Difficulty of Measuring IR SEDs at $z>5$}

In Section 4.1, we show that knowledge of the shape of the IR SED is crucial to measure basic IR properties like the total luminosity $L_{\mathrm{FIR}}$. Furthermore, it enables us to study in detail the ISM properties of galaxies such as their temperature and optical depth, which tell us about the current star formation conditions in these galaxies. As pointed out earlier, the IR SEDs of $z>5$ galaxies are very poorly constrained, most of the time by just one data point at $158 \mu \mathrm{m}$, if at all. Measurement of DSFGs at $z=5-6$ reveals increased $T$ and thus warm SED shapes compared to galaxies at $z<4$ (Riechers et al. 2013, 2014; Ma et al. 2016; Strandet et al. 2016). The first estimates of $T$ for two normal $z \sim 5.5$ galaxies reveal two very different temperatures, indicative of large variations in the SED shape of high-redshift galaxies (Pavesi et al. 2016). However, these measurements are uncertain as they only use wavelength data redward of [C II]. One way to investigate the diversity of IR SEDs of high-redshift galaxies statistically is to use larger samples of low-redshift analogues of these galaxies. This, however, might be dangerous as we do not know the degree to which analogues actually represent the high-redshift galaxy population as a whole. Direct measurements of galaxies at high redshifts therefore seems to be the preferred strategy. Due to the faintness of these galaxies, this is a difficult endeavor and hence an optimal and effective observing strategy is needed.

In the following, we outline a possible observing strategy in order to better constrain the IR SEDs (specifically constrained by the luminosity-weighted temperature $T$ ) of $z \sim 6$ galaxies. Since observations in the IR are expensive, we focus in particular on minimizing the total observing time and maximizing the science output.

\section{A.1. Theoretical Calculations}

To measure the temperature of high-redshift galaxies via continuum measurements, we choose wavelengths close to bright FIR emission lines ([O I] at $63 \mu \mathrm{m}$, [O III] at $88 \mu \mathrm{m}$, [N II] at 122 and $205 \mu \mathrm{m}$, and [C II] at $158 \mu \mathrm{m})$. With this strategy, any ALMA observations will produce line diagnostics in addition to FIR continuum measurements to further investigate the ISM of these galaxies (e.g., Pavesi et al. 2016). Figure 15 shows the observability of these FIR emission lines with ALMA as a function of redshift. The color and width of the bands for each FIR emission line corresponds to the atmospheric transmission in percent assuming a precipitable water vapor of $1 \mathrm{~mm}$. An efficient strategy is to look for the lowest redshift (to have a reasonable sample of spectroscopically confirmed galaxies) at which most of the FIR lines are observable with ALMA. It turns out that the redshift range $5.82<z<5.96$ fulfills this criteria with Band 5 being operational in ALMA cycle 5. Note that $z \sim 6.1$ and $z \sim 6.3$ are other possible windows at which all five emission lines are observable. If we are only interested in the highest transmission wavelengths $(122,158$, and $205 \mu \mathrm{m})$, all redshifts above $z \sim 5.9$ are a good choice.

The left-side panel in Figure 16 shows FIR continuum flux ratios around these FIR emission lines as a function of the luminosity-weighted temperature derived from a graybody + mid-IR power-law parametrization by Casey (2012). We choose to use the ratios with respect to rest-frame $158 \mu \mathrm{m}$, which has a high atmospheric transmission $(93 \%$ at $z \sim 6)$ and is well constrained by existing data sets in terms of detections and limits. In the following, we refer to $158 \mu \mathrm{m}$ as the "primary wavelength" and to the other wavelengths $(63,88,122$, and $205 \mu \mathrm{m})$ as the "secondary wavelengths." The width of the bands visualizes the model uncertainty (90\% confidence interval) of the continuum flux ratio for a large range in emissivities $\left(0.5<\beta_{\text {IR }}<2.5\right)$ and mid-IR power-law slopes $(1<\alpha<3)$. We note that the relation between continuum flux ratio and temperature does not significantly depend on $\alpha$ and $\beta_{\mathrm{IR}}$ and is primarily dependent on the assumed temperature $T$ (see also Section 4.1). The black points with error bars show simulated data points with fluxes observed at different depths for comparison. The continuum flux ratio $F_{158 \mu \mathrm{m}} / F_{63 \mu \mathrm{m}}$ would be the ideal choice to quantify the temperature because of its steep dependence on temperature. However, the atmospheric transparency for $z \sim 5.9$ at the observed wavelength of $63 \mu \mathrm{m}$ is lowest with $38 \%$ compared to $40 \%, 82 \%$, and $94 \%$ for $88 \mu \mathrm{m}, 122 \mu \mathrm{m}$, and $205 \mu \mathrm{m}$, respectively. The right-side panel in Figure 16 shows this more qualitatively. Each subpanel corresponds to a continuum flux ratio and shows the needed depth of the flux measurement (in $\sigma$ ) to reach a certain $\sigma$ of significance to distinguish three different pairs of temperatures. The calculation of the latter combines the model uncertainty at a given temperature with the measurement uncertainty (corresponding to the $y$-axis). The $F_{158 \mu \mathrm{m}} / F_{63 \mu \mathrm{m}}$ ratio gives the best turnout for a given depth of flux measurement. All considered temperatures can be distinguished at $>2 \sigma(>3 \sigma)$ significance for a $10 \sigma(20 \sigma)$ detection at $63 \mu \mathrm{m}$. For $F_{158 \mu \mathrm{m}} / F_{88 \mu \mathrm{m}}$, temperatures of $30 \mathrm{~K}$ and $60 \mathrm{~K}$ can be distinguished at $2 \sigma-2.5 \sigma$ significance for a $6 \sigma$ detection at $88 \mu \mathrm{m}$, while for $F_{158 \mu \mathrm{m}} / F_{122 \mu \mathrm{m}}$, a $>15 \sigma$ detection in $122 \mu \mathrm{m}$ 
IR emission (dust emission)

UV emission (unobscured star forming regions)

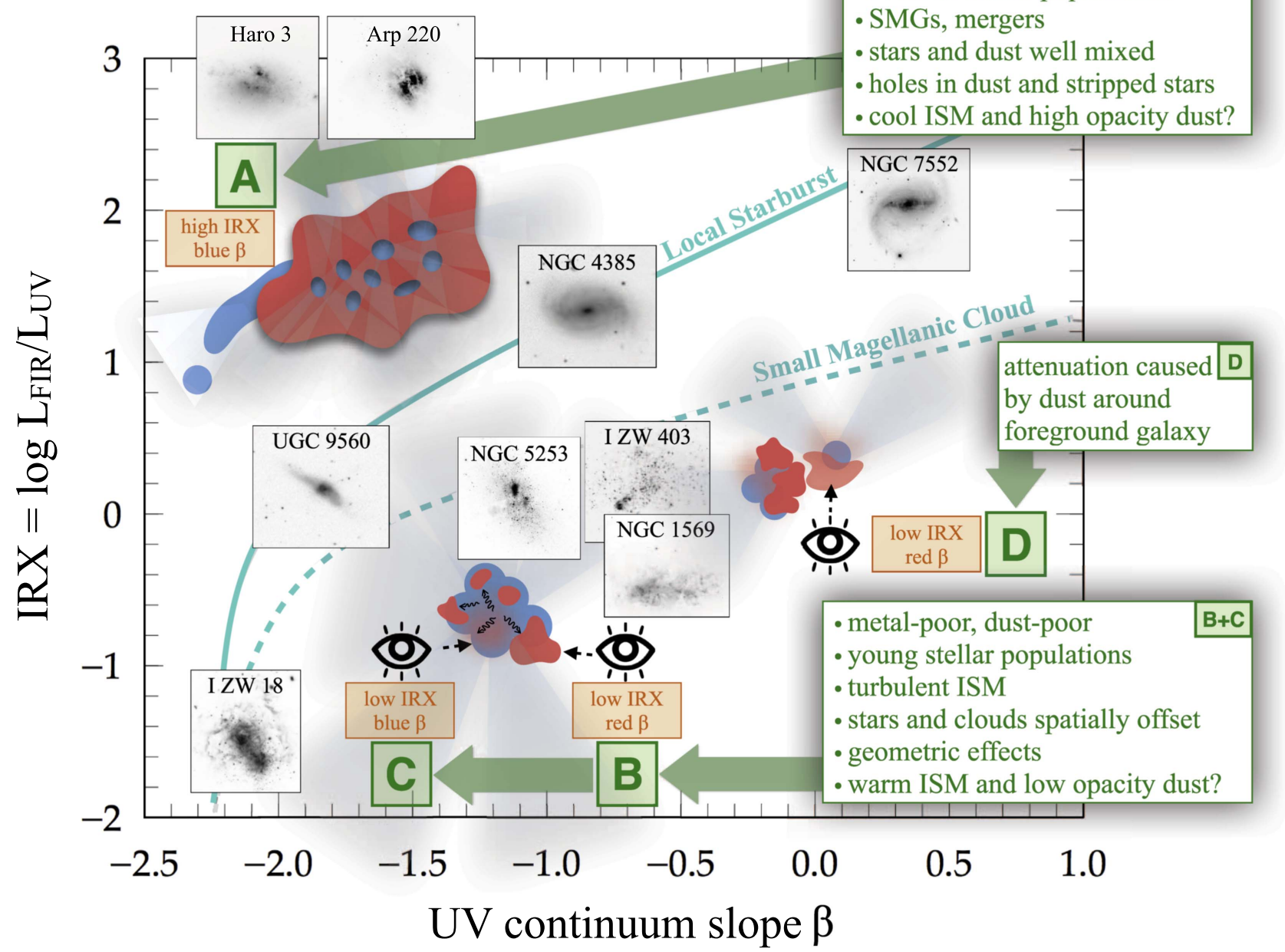

Figure 14. Schematic of different populations of galaxies at $z \sim 5.5$ on the IRX $-\beta$ diagram and their likely properties suggested by [C II] emission, FIR continuum, UV continuum, Spitzer colors, and UV spectroscopy (Sections 4.2, 4.3, and 4.4). The insets show multicolor images of local galaxies placed at their approximate position on the IRX - $\beta$ diagram (e.g., Gil de Paz et al. 2007). The IR emission (dust) is color-coded in red, and the UV emission (unobscured star-forming regions) in blue. Configuration A: galaxies with high IRX values and blue UV colors are likely dust rich, metal rich, and consist of evolved stellar populations. The stars and gas are well-mixed in a spatially compact configuration. The blue UV colors could be due to holes in the dust cover, tidally stripped young stars, or faint blue satellite galaxies. Configurations B and C: galaxies with low IRX values and a range of UV colors have dust-poor and metal-poor young stellar populations. The dust clouds and star-forming regions are spatially separated in the turbulent ISM of these galaxies due to stellar radiation pressure, mergers, and gas inflows. Our proposed model (Section 4.5) can explain the low IRX values of these galaxies. Such a configuration causes obscured sightlines (B) as well as clear sightlines (C), and hence the UV color depends significantly on geometry and viewing angle. This way, red UV colors at low IRX values can be reached. Configuration D: in some cases, low IRX and red UV colors could be created by the attenuation of the UV light of a background galaxy by the thin layer of dust that is expelled into the circumgalactic medium around a foreground galaxy (Section 4.6).

is necessary to reach the same significance. Finally, the $F_{158 \mu \mathrm{m}} / F_{205 \mu \mathrm{m}}$ versus temperature relation is too shallow to separate temperatures at $T>30 \mathrm{~K}$ with a significance of more than $1 \sigma$ for any observational depth. The latter is the reason why the temperature estimates by Pavesi et al. (2016) have such a large quoted uncertainty.

\section{A.2. A Fiducial Survey at $z=5.9$}

The final success in measuring temperature depends on (i) the ability to reach the flux ratio for a given temperature (the limiting flux ratio $\xi$ ) and (ii) the ability to split different temperatures (as discussed above). Figure 17 demonstrates these points by combining the previous results with relative integration time calculations for a fiducial redshift of $z=5.9$.
The panels show the reached flux ratio above the limiting flux ratio as a function of integration in the secondary wavelengths with respect to the integration time necessary for a $10 \sigma$ detection in $158 \mu \mathrm{m}$. Note that $\xi<1$ only results in an upper limit in temperature for all flux ratios except for $F_{158 \mu \mathrm{m}} / F_{205 \mu \mathrm{m}}$, where it results in a lower temperature limit (see the left panel of Figure 16). The horizontal panels show the four different flux ratios while the sets of panels (arranged vertically) show different depths for the observations at the secondary wavelengths $(5 \sigma, 10 \sigma$, and $15 \sigma)$. The width of the curves (showing temperatures of 30,60 , and $90 \mathrm{~K})^{23}$ include the model and measurement uncertainties. In summary, we note the following.

\footnotetext{
$\overline{{ }^{23} \text { Corresponding to } 20,35 \text {, and } 50 \mathrm{~K} \text { in } T_{\text {peak }}}$.
} 
Atmospheric Transmission [\%]

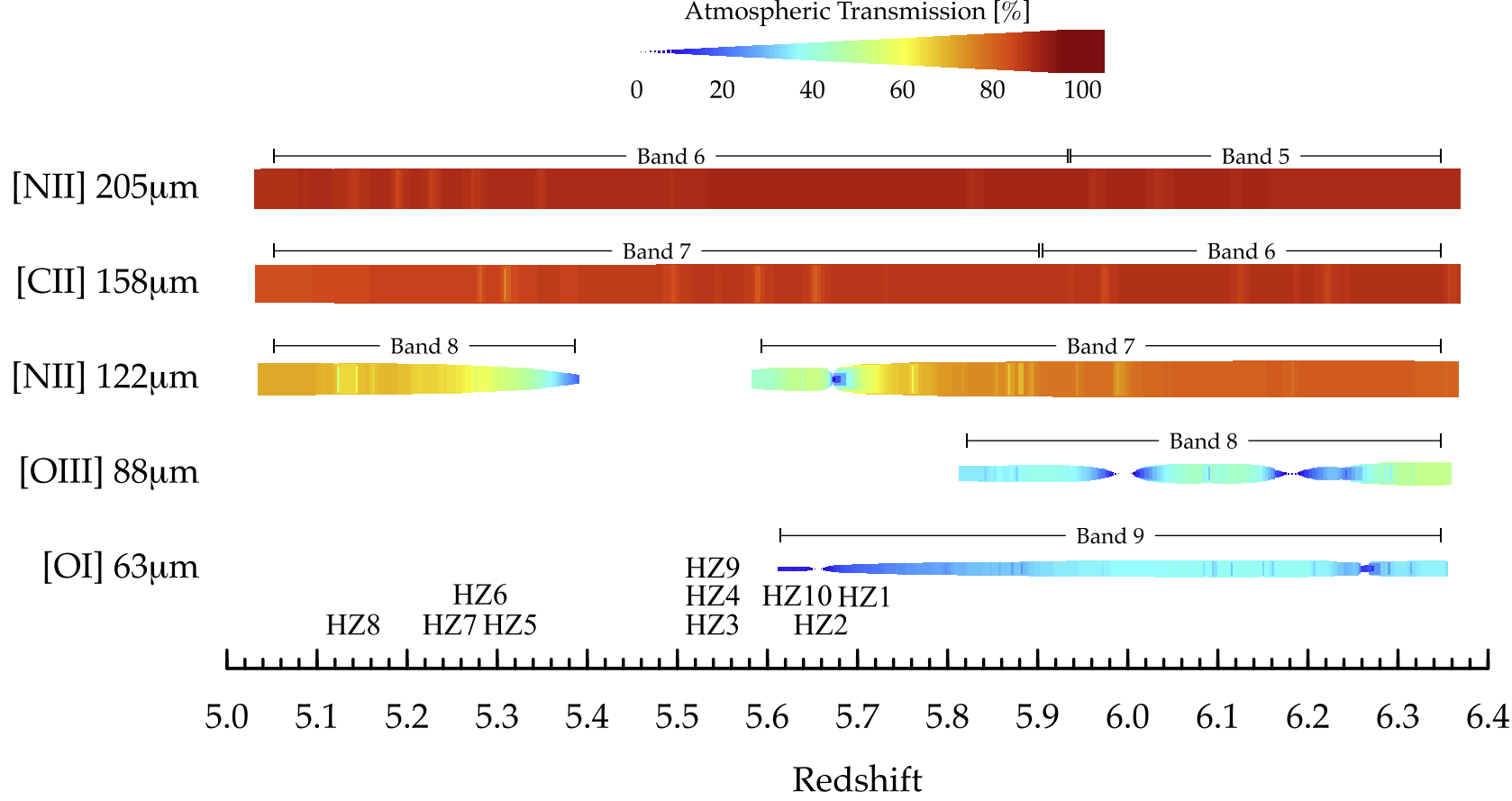

Figure 15. Observability of FIR emission lines ([O I], [O III], [N II], and [C II]) with ALMA as a function of redshift. The atmospheric transmission (assuming a precipitable water vapor column density of $1 \mathrm{~mm}$ ) is indicated in colors and the width of the bars. We also indicate the ALMA bands in which the lines can be observed. The redshift range $5.82<z<5.96$ is the lowest for which all FIR lines can be observed at a reasonable atmospheric transmission. Spectroscopic samples become scarce at higher redshifts. The redshifts of the $\mathrm{C} 15$ galaxies are also indicated for reference.
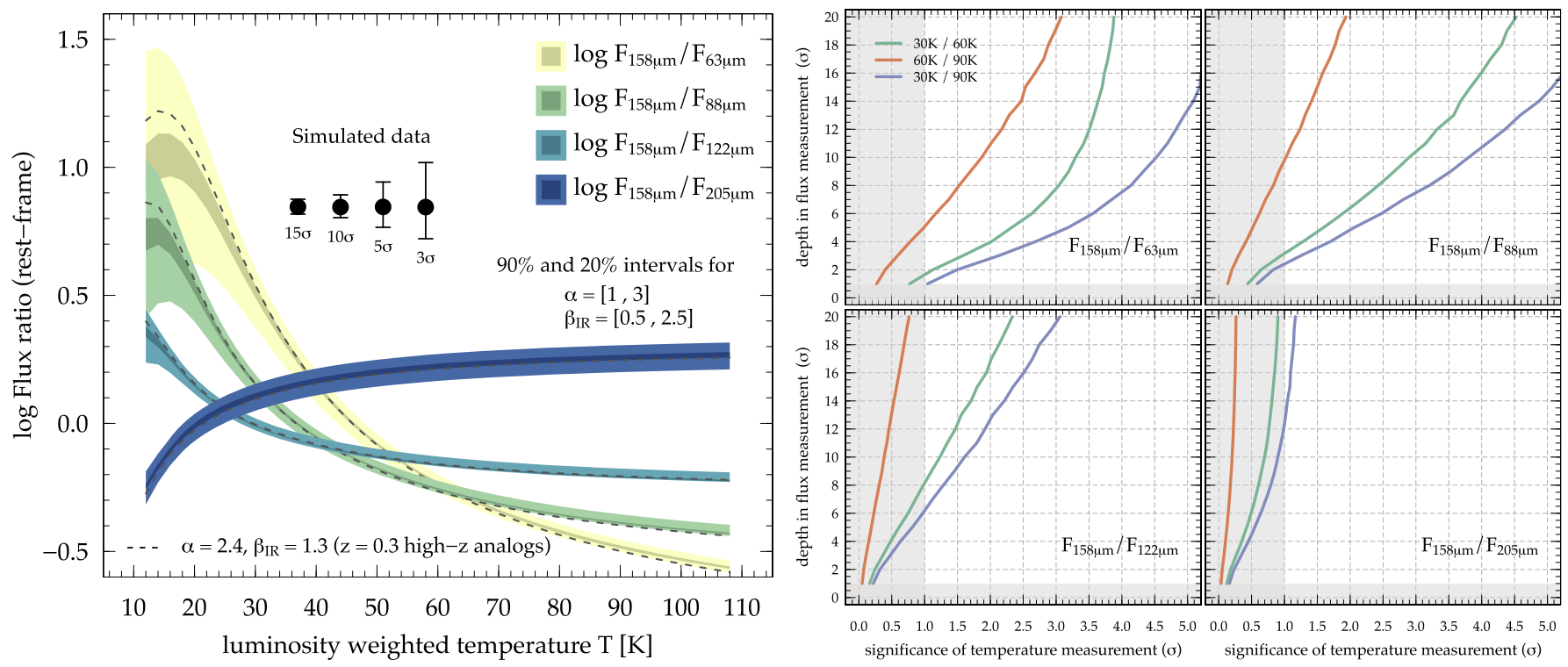

Figure 16. Left panel: various flux ratios (with respect to $158 \mu \mathrm{m}$ ) computed for a graybody + MIR power law (Casey 2012) as a function of luminosity-weighted temperature. The width of the curves correspond to the model uncertainties assuming a range in MIR power-law $\alpha$ and emissivity $\left(\beta_{\text {IR }}\right)$ as indicated. The dotted line represents the mean parameters of our $z \sim 0.3$ analogues of high- $z$ galaxies. The black symbols show the uncertainties in the flux ratios for detections of different $\sigma$ significance. Right panels: necessary depth of flux observations $(\sigma)$ in $63,88,122$, and $205 \mu \mathrm{m}$ (four subpanels) to reach a temperature measurement of a given $\sigma$ confidence to distinguish among three different pairs of temperatures (color-coded). Temperatures above $30 \mathrm{~K}$ are not possible to distinguish at $>1 \sigma$ with the $F_{158 \mu \mathrm{m}} / F_{205 \mu \mathrm{m}}$ flux ratio even with a $20 \sigma$ observation at $205 \mu \mathrm{m}$.

1. Temperatures cannot be distinguished above $T=60 \mathrm{~K}$ with more than $1 \sigma$ significance using the $F_{158 \mu \mathrm{m}} / F_{122 \mu \mathrm{m}}$ and $F_{158 \mu \mathrm{m}} / F_{205 \mu \mathrm{m}}$ ratios at flux measurement depths corresponding to less than $15 \sigma$ significance.
2. Temperatures can be confined at $T<30 \mathrm{~K}$ or $T>$ $30 \mathrm{~K}$ at $>1.5 \sigma$ for a $10 \sigma$ detection in $122 \mu \mathrm{m}$ or $205 \mu \mathrm{m}$ within $1-2$ times the integration time in $158 \mu \mathrm{m}$ (with $10 \sigma$ ). 

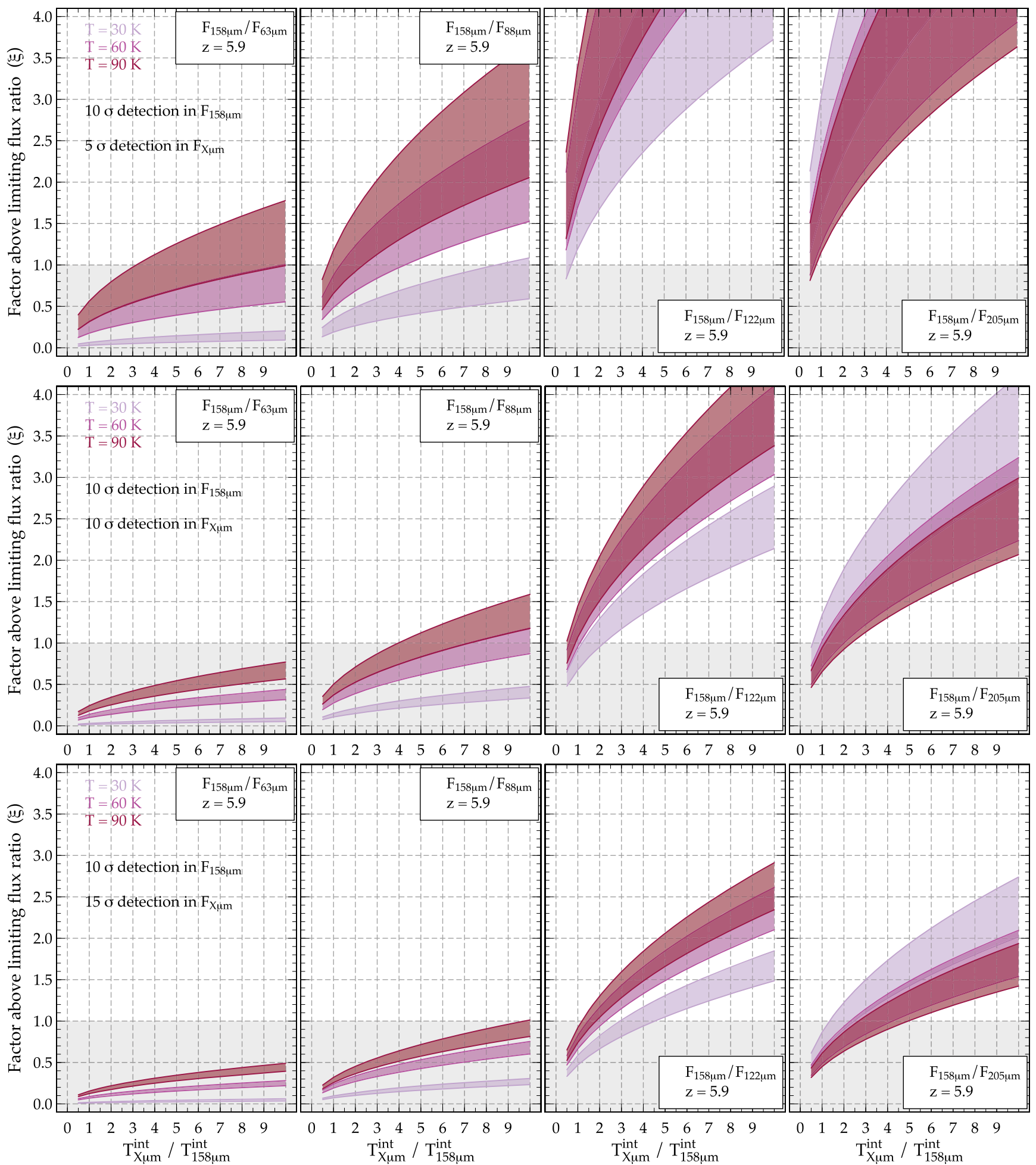

Figure 17. Within each of the three sets of panels, the subpanels show the significance to distinguish temperatures of 30,60 , and $90 \mathrm{~K}$ with flux ratios $F_{158} \mu \mathrm{m} / F_{X \mu \mathrm{m}}$, where $X$ denotes the "secondary bands" $(63,88,122$, and $205 \mu \mathrm{m}$ from left to right, respectively), as a function of integration time in the secondary bands with respect to the integration time at $158 \mu \mathrm{m}(10 \sigma$ detection). The width of the curves include model uncertainties (see Figure 16) as well as measurement uncertainties, which are varied between the three sets of panels $(5 \sigma, 10 \sigma$, and $15 \sigma$ detection in the secondary bands from top to bottom). Temperatures can only be cleanly separated if the curves are not overlapping. Furthermore, the $y$-axis shows the factor above the flux ratio, $\xi$, that can be observed in a given configuration. Thus, if $\xi<1$, the depth of an observation is not sufficient to measure a flux ratio at a given temperature as shown in Figure 16. It is difficult to distinguish temperatures between $T=60 \mathrm{~K}$ and $T=90 \mathrm{~K}$, because the temperature-flux ratio relations are too shallow for 122 and $205 \mu \mathrm{m}$ even for very high $\sigma$ detections, and, on the other hand, a too long integration time at 63 and $88 \mu \mathrm{m}$ is needed to measure flux ratios at all, and at the same time providing a $>5 \sigma$ detection to distinguish the temperatures. In contrast, temperatures between $T=30 \mathrm{~K}$ and $T=60 \mathrm{~K}$ are easy to distinguish with $F_{158 \mu \mathrm{m}} / F_{122 \mu \mathrm{m}}$ and $F_{158 \mu \mathrm{m}} / F_{205 \mu \mathrm{m}}$ flux ratios and $>10 \sigma$ detection at 122 and $205 \mu \mathrm{m}$. 

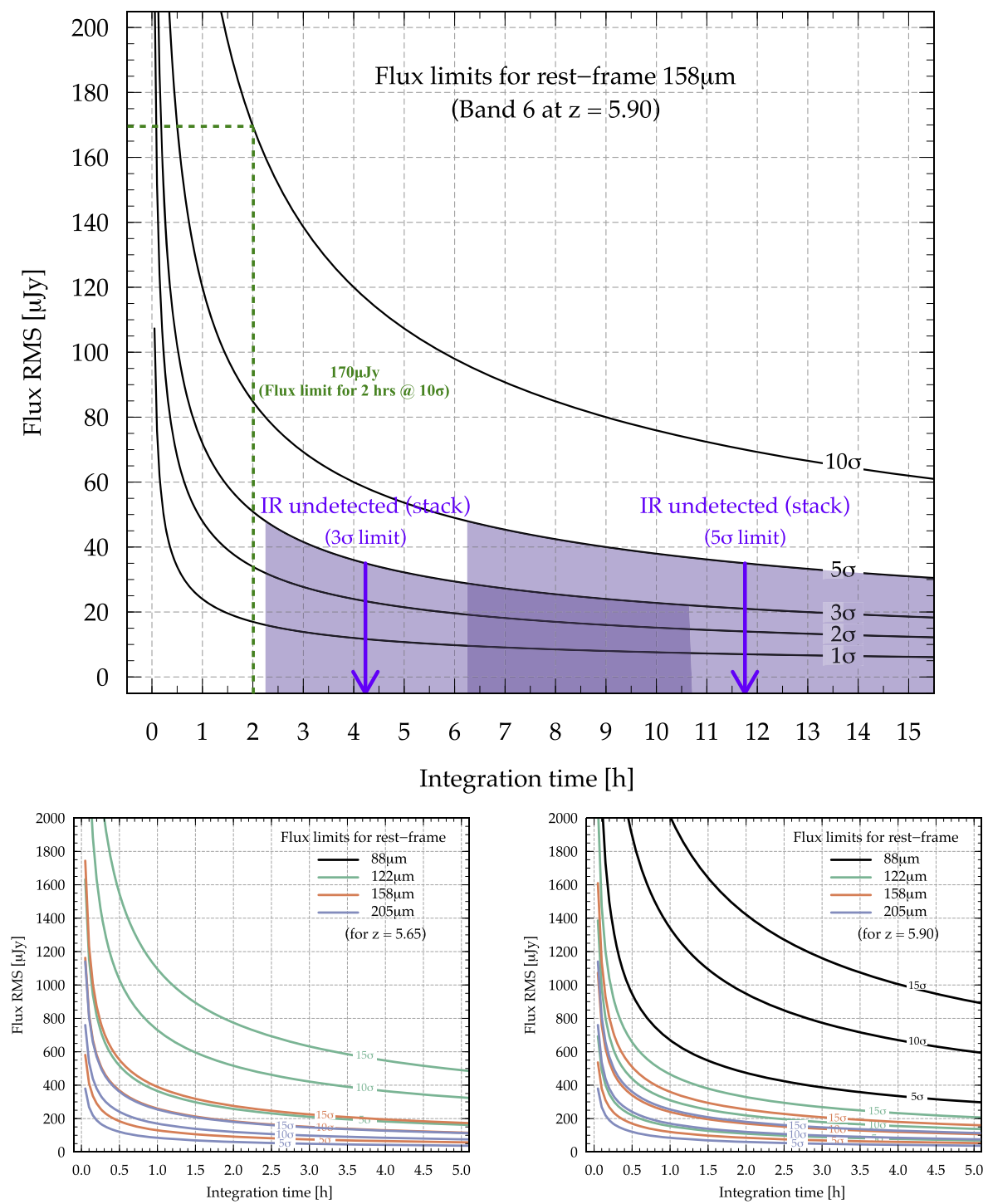
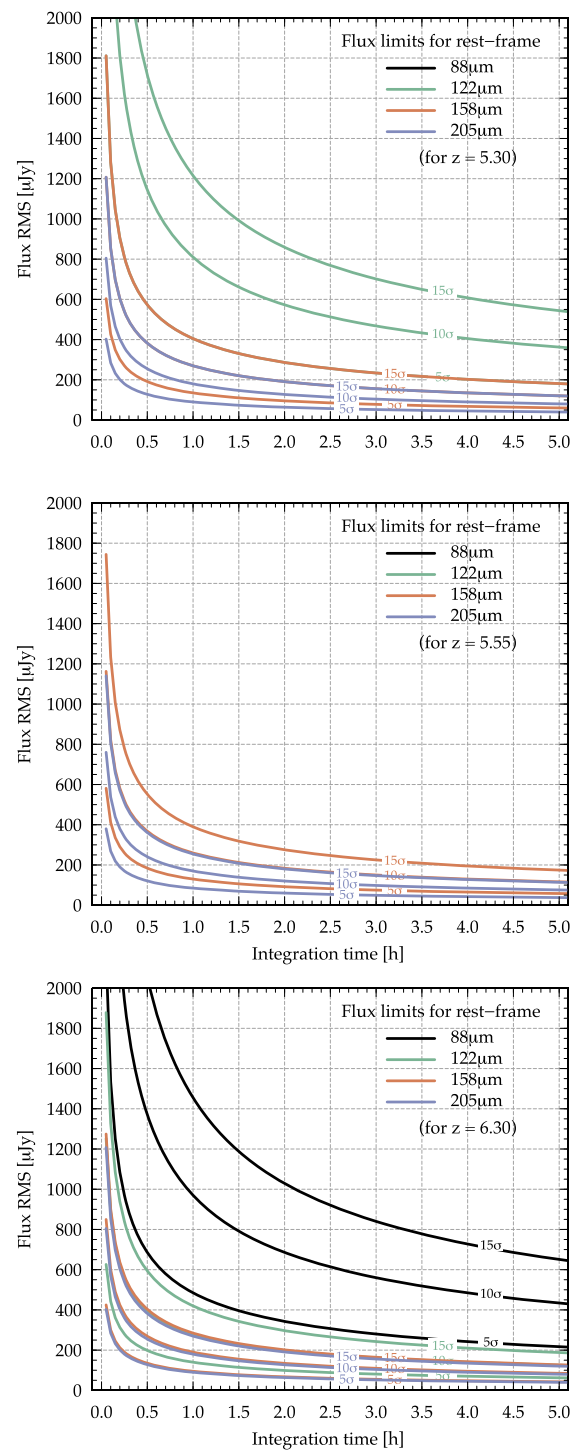

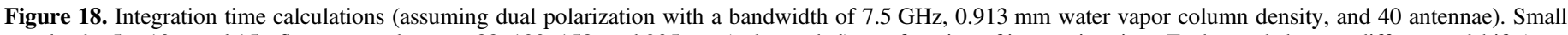

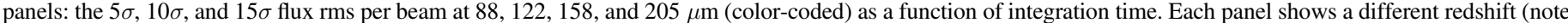

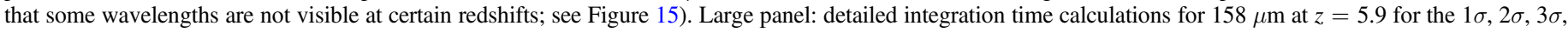

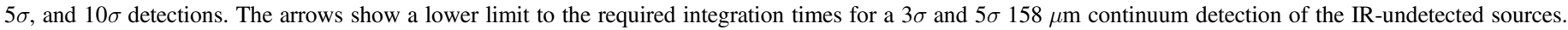

3. The measurement of temperatures with the $F_{158 \mu \mathrm{m}} / F_{63 \mu \mathrm{m}}$ and $F_{158 \mu \mathrm{m}} / F_{88 \mu \mathrm{m}}$ ratios is not feasible within a reasonable amount of time (less than 10 times the integration time in $158 \mu \mathrm{m})$.

4. Therefore, the optimal observing strategy to study the temperature of $z \sim 6$ galaxies is to target rest-frame $158 \mu \mathrm{m}$ and $122 \mu \mathrm{m}$ (or $205 \mu \mathrm{m}$ if $122 \mu \mathrm{m}$ is affected by low transmission, but with a significantly decreased success rate), thereby requiring a depth of at least $10 \sigma$.

After setting up the framework, we now proceed to establish integration times and flux limits for which temperatures can be measured efficiently. Figure 18 shows the estimated flux root mean square (rms) limits at a given $\sigma$ significance as a function of integration time for different wavelengths and redshifts. We assume a dual polarization set-up with a bandwidth of $7.5 \mathrm{GHz}$, precipitable water vapor column density of $0.913 \mathrm{~mm}, 40$ antennae, and a resolution of $1^{\prime \prime}$ in the following. The calculations are done for objects at $\delta(2000)=+02: 00: 00$ observed with ALMA, and the integration times do not include overheads. The large panel shows a more detailed graph for $158 \mu \mathrm{m}$ at $z=5.9$. An integration time of $2 \mathrm{hr}$ would result in a $10 \sigma 158 \mu \mathrm{m}$ continuum flux sensitivity of $170 \mu \mathrm{Jy}$ per beam, which is similar to the flux observed in HZ4 (see Table 6). To distinguish temperatures of 30 and $60 \mathrm{~K}$ with $F_{158 \mu \mathrm{m}} / F_{122 \mu \mathrm{m}}$ for such a source at $z=5.9$ with a significance of $1 \sigma$ (for which a $10 \sigma$ detection at $122 \mu \mathrm{m}$ is necessary), we have to double the integration time at $158 \mu \mathrm{m}$, thus $4 \mathrm{hr}$ at $122 \mu \mathrm{m}$ (see Figure 17). Alternatively, a $2 \times 4=8 \mathrm{hr}$ integration (corresponding to $15 \sigma$ at $122 \mu \mathrm{m}$ ) will result in a significance of $2 \sigma$ to distinguish $T=30 \mathrm{~K}$ and $T=60 \mathrm{~K}$. The significance increases by roughly $30 \%-50 \%$ for temperatures of $T=30 \mathrm{~K}$ and $T=90 \mathrm{~K}$.

\section{A.3. Temperature Measurement of Existing Sources Detected in [C II]}

C15 provides four of the IR-brightest $\sim L^{*}$ galaxies at $z \sim 5.5$ that can be followed up with our method to verify our inferred 
Table 6

Expected Photometry for High- $z$ Galaxies Predicted from Their $158 \mu$ m Flux and $1.5<\alpha<2.5,1<\beta_{\text {IR }}<2$, and $60 \mathrm{~K}<T<90 \mathrm{~K}\left(35 \mathrm{~K}<T_{\text {peak }}<50 \mathrm{~K}\right)$

\begin{tabular}{|c|c|c|c|c|c|c|c|c|c|c|c|}
\hline Name & $z$ & $\log \left(L_{\mathrm{FIR}} / L_{\odot}\right)$ & $\begin{array}{r}63 \mu \mathrm{m} \\
(\mu \mathrm{Jy})\end{array}$ & $\begin{array}{r}88 \mu \mathrm{m} \\
(\mu \mathrm{Jy})\end{array}$ & $\begin{array}{r}110 \mu \mathrm{m} \\
(\mu \mathrm{Jy})\end{array}$ & $\begin{array}{r}122 \mu \mathrm{m} \\
(\mu \mathrm{Jy})\end{array}$ & $\begin{array}{c}158 \mu \mathrm{m}^{\mathrm{a}} \\
(\mu \mathrm{Jy})\end{array}$ & $\begin{array}{r}190 \mu \mathrm{m} \\
(\mu \mathrm{Jy})\end{array}$ & $\begin{array}{r}205 \mu \mathrm{m} \\
(\mu \mathrm{Jy})\end{array}$ & $\begin{array}{r}220 \mu \mathrm{m} \\
(\mu \mathrm{Jy})\end{array}$ & Reference $^{b}$ \\
\hline HZ10 & 5.6566 & $12.58_{-0.25}^{+0.22}$ & $3100_{-649}^{+583}$ & $2773_{-319}^{+268}$ & $2234_{-136}^{+120}$ & $1947_{-81}^{+74}$ & $\left(1261_{-0}^{+0}\right)$ & $858_{-28}^{+31}$ & $722_{-37}^{+38}$ & $608_{-39}^{+45}$ & 1 \\
\hline HZ9 & 5.5410 & $12.18_{-0.25}^{+0.22}$ & $1269_{-266}^{+239}$ & $1135_{-130}^{+110}$ & $914_{-56}^{+49}$ & $797_{-33}^{+30}$ & $\left(516_{-0}^{+0}\right)$ & $351_{-11}^{+13}$ & $296_{-15}^{+15}$ & $249_{-16}^{+19}$ & 1 \\
\hline HZ6 (all) & 5.2928 & $11.78_{-0.25}^{+0.22}$ & $541_{-113}^{+102}$ & $484_{-56}^{+47}$ & $390_{-24}^{+21}$ & $340_{-14}^{+13}$ & $\left(220_{-0}^{+0}\right)$ & $150_{-5}^{+5}$ & $126_{-6}^{+7}$ & $106_{-7}^{+8}$ & 2 \\
\hline WMH5 & 6.0695 & $11.87_{-0.25}^{+0.22}$ & $536_{-112}^{+101}$ & $479_{-55}^{+46}$ & $386_{-24}^{+21}$ & $337_{-14}^{+13}$ & $\left(218_{-0}^{+0}\right)$ & $148_{-5}^{+5}$ & $125_{-6}^{+7}$ & $105_{-7}^{+8}$ & 3 \\
\hline HZ4 & 5.5440 & $11.78_{-0.25}^{+0.22}$ & $497_{-104}^{+93}$ & $444_{-51}^{+43}$ & $358_{-22}^{+19}$ & $312_{-13}^{+12}$ & $\left(202_{-0}^{+0}\right)$ & $137_{-4}^{+5}$ & $116_{-6}^{+6}$ & $97_{-6}^{+7}$ & 1 \\
\hline HZ6b & 5.2928 & $11.52_{-0.25}^{+0.22}$ & $295_{-62}^{+56}$ & $264_{-30}^{+25}$ & $213_{-13}^{+11}$ & $185_{-8}^{+7}$ & $\left(120_{-0}^{+0}\right)$ & $82_{-3}^{+3}$ & $69_{-3}^{+4}$ & $58_{-4}^{+4}$ & 2 \\
\hline HZ6c & 5.2928 & $11.44_{-0.25}^{+0.22}$ & $246_{-51}^{+46}$ & $220_{-25}^{+21}$ & $177_{-11}^{+9}$ & $154_{-6}^{+6}$ & $\left(100_{-0}^{+0}\right)$ & $68_{-2}^{+2}$ & $57_{-3}^{+3}$ & $48_{-3}^{+4}$ & 2 \\
\hline IDX34 & 7.4910 & $<11.66_{-0.25}^{+0.22}$ & $<246_{-51}^{+46}$ & $<220_{-25}^{+21}$ & $<177_{-11}^{+9}$ & $<154_{-6}^{+6}$ & $\left(<100_{-0}^{+0}\right)$ & $<68_{-2}^{+2}$ & $<57_{-3}^{+3}$ & $<48_{-3}^{+4}$ & 4 \\
\hline ID04 & 6.8670 & $11.52_{-0.25}^{+0.22}$ & $202_{-42}^{+38}$ & $180_{-21}^{+17}$ & $145_{-9}^{+8}$ & $127_{-5}^{+5}$ & $\left(82_{-0}^{+0}\right)$ & $56_{-2}^{+2}$ & $47_{-2}^{+2}$ & $40_{-3}^{+3}$ & 4 \\
\hline ID52 & 6.0180 & $<11.37_{-0.25}^{+0.22}$ & $<172_{-36}^{+32}$ & $<154_{-18}^{+15}$ & $<124_{-8}^{+7}$ & $<108_{-4}^{+4}$ & $\left(<70_{-0}^{+0}\right)$ & $<48_{-2}^{+2}$ & $<40_{-2}^{+2}$ & $<34_{-2}^{+3}$ & 4 \\
\hline ID30 & 6.8540 & $<11.42_{-0.25}^{+0.22}$ & $<160_{-33}^{+30}$ & $<143_{-16}^{+14}$ & $<115_{-7}^{+6}$ & $<100_{-4}^{+4}$ & $\left(<65_{-0}^{+0}\right)$ & $<44_{-1}^{+2}$ & $<37_{-2}^{+2}$ & $<31_{-2}^{+2}$ & 4 \\
\hline ID02 & 7.9140 & $<11.43_{-0.25}^{+0.22}$ & $<135_{-28}^{+25}$ & $<121_{-14}^{+10}$ & $<97_{-6}^{+5}$ & $<85_{-4}^{+3}$ & $\left(<55_{-0}^{+0}\right)$ & $<37_{-1}^{+1}$ & $<32_{-2}^{+2}$ & $<27_{-2}^{+2}$ & 4 \\
\hline $\mathrm{HZ3}$ & 5.5416 & $<11.18_{-0.25}^{+0.22}$ & $<125_{-26}^{+24}$ & $<112_{-13}^{+11}$ & $<90_{-6}^{+5}$ & $<79_{-3}^{+3}$ & $\left(<51_{-0}^{+0}\right)$ & $<35_{-1}^{+1}$ & $<29_{-1}^{+2}$ & $<25_{-2}^{+2}$ & 1 \\
\hline CLM1 & 6.1657 & $11.18_{-0.25}^{+0.22}$ & $108_{-23}^{+20}$ & $97_{-11}^{+9}$ & $78_{-5}^{+4}$ & $68_{-3}^{+3}$ & $\left(44_{-0}^{+0}\right)$ & $30_{-1}^{+1}$ & $25_{-1}^{+1}$ & $21_{-1}^{+2}$ & 3 \\
\hline ID41 & 6.3460 & $<11.20_{-0.25}^{+0.22}$ & $<108_{-23}^{+20}$ & $<97_{-11}^{+9}$ & $<78_{-5}^{+4}$ & $<68_{-3}^{+3}$ & $\left(<44_{-0}^{+0}\right)$ & $<30_{-1}^{+1}$ & $<25_{-1}^{+1}$ & $<21_{-1}^{+2}$ & 4 \\
\hline ID09 & 6.0240 & $<11.15_{-0.25}^{+0.22}$ & $<103_{-22}^{+19}$ & $<92_{-11}^{+9}$ & $<74_{-5}^{+4}$ & $<65_{-3}^{+2}$ & $\left(<42_{-0}^{+0}\right)$ & $<29_{-1}^{+1}$ & $<24_{-1}^{+1}$ & $<20_{-1}^{+2}$ & 4 \\
\hline ID14 & 6.7510 & $<11.22_{-0.25}^{+0.22}$ & $<103_{-22}^{+19}$ & $<92_{-11}^{+9}$ & $<74_{-5}^{+4}$ & $<65_{-3}^{+2}$ & $\left(<42_{-0}^{+0}\right)$ & $<29_{-1}^{+1}$ & $<24_{-1}^{+1}$ & $<20_{-1}^{+2}$ & 4 \\
\hline ID38 & 6.5930 & $<11.20_{-0.25}^{+0.22}$ & $<103_{-22}^{+19}$ & $<92_{-11}^{+9}$ & $<74_{-5}^{+4}$ & $<65_{-3}^{+2}$ & $\left(<42_{-0}^{+0}\right)$ & $<29_{-1}^{+1}$ & $<24_{-1}^{+1}$ & $<20_{-1}^{+2}$ & 4 \\
\hline IDX25 & 6.3570 & $<11.16_{-0.25}^{+0.22}$ & $<98_{-21}^{+19}$ & $<88_{-10}^{+8}$ & $<71_{-4}^{+4}$ & $<62_{-3}^{+2}$ & $\left(<40_{-0}^{+0}\right)$ & $<27_{-1}^{+1}$ & $<23_{-1}^{+1}$ & $<19_{-1}^{+1}$ & 4 \\
\hline ID44 & 7.3600 & $<11.25_{-0.25}^{+0.22}$ & $<98_{-21}^{+19}$ & $<88_{-10}^{+8}$ & $<71_{-4}^{+4}$ & $<62_{-3}^{+2}$ & $\left(<40_{-0}^{+0}\right)$ & $<27_{-1}^{+1}$ & $<23_{-1}^{+1}$ & $<19_{-1}^{+1}$ & 4 \\
\hline ID49 & 6.0510 & $<11.13_{-0.25}^{+0.22}$ & $<98_{-21}^{+19}$ & $<88_{-10}^{+8}$ & $<71_{-4}^{+4}$ & $<62_{-3}^{+2}$ & $\left(<40_{-0}^{+0}\right)$ & $<27_{-1}^{+1}$ & $<23_{-1}^{+1}$ & $<19_{-1}^{+1}$ & 4 \\
\hline $\mathrm{HZ7}$ & 5.2532 & $<10.99_{-0.25}^{+0.22}$ & $<89_{-19}^{+17}$ & $<79_{-9}^{+8}$ & $<64_{-4}^{+3}$ & $<56_{-2}^{+2}$ & $\left(<36_{-0}^{+0}\right)$ & $<24_{-1}^{+1}$ & $<21_{-1}^{+1}$ & $<17_{-1}^{+1}$ & 1 \\
\hline ID27 & 7.5750 & $11.20_{-0.25}^{+0.22}$ & $84_{-17}^{+16}$ & $75_{-9}^{+7}$ & $60_{-4}^{+3}$ & $53_{-2}^{+2}$ & $\left(34_{-0}^{+0}\right)$ & $23_{-1}^{+1}$ & $19_{-1}^{+1}$ & $16_{-1}^{+1}$ & 4 \\
\hline HZ5 & 5.3089 & $<10.95_{-0.25}^{+0.22}$ & $<79_{-16}^{+115}$ & $<70_{-8}^{+7}$ & $<57_{-3}^{+3}$ & $<49_{-2}^{+2}$ & $\left(<32_{-0}^{+0}\right)$ & $<22_{-1}^{+1}$ & $<18_{-1}^{+1}$ & $<15_{-1}^{+1}$ & 1 \\
\hline $\mathrm{HZ5a}$ & 5.3089 & $<10.95_{-0.25}^{+0.22}$ & $<79_{-16}^{+15}$ & $<70_{-8}^{+7}$ & $<57_{-3}^{+3}$ & $<49_{-2}^{+2}$ & $\left(<32_{-0}^{+0}\right)$ & $<22_{-1}^{+1}$ & $<18_{-1}^{+1}$ & $<15_{-1}^{+1}$ & 1 \\
\hline HZ1 & 5.6886 & $<10.96_{-0.25}^{+0.22}$ & $<74_{-15}^{+14}$ & $<66_{-8}^{+6}$ & $<53_{-3}^{+3}$ & $<46_{-2}^{+2}$ & $\left(<30_{-0}^{+0}\right)$ & $<20_{-1}^{+1}$ & $<17_{-1}^{+1}$ & $<14_{-1}^{+1}$ & 1 \\
\hline HZ6a & 5.2928 & $10.92_{-0.25}^{+0.22}$ & $74_{-15}^{+14}$ & $66_{-8}^{+6}$ & $53_{-3}^{+3}$ & $46_{-2}^{+2}$ & $\left(30_{-0}^{+0}\right)$ & $20_{-1}^{+1}$ & $17_{-1}^{+1}$ & $14_{-1}^{+1}$ & 2 \\
\hline HZ8 & 5.1533 & $<10.90_{-0.25}^{+0.22}$ & $<74_{-15}^{+14}$ & $<66_{-8}^{+6}$ & $<53_{-3}^{+3}$ & $<46_{-2}^{+2}$ & $\left(<30_{-0}^{+0}\right)$ & $<20_{-1}^{+1}$ & $<17_{-1}^{+1}$ & $<14_{-1}^{+1}$ & 1 \\
\hline HZ8W & 5.1532 & $<10.90_{-0.25}^{+0.22}$ & $<74_{-15}^{+14}$ & $<66_{-8}^{+6}$ & $<53_{-3}^{+3}$ & $<46_{-2}^{+2}$ & $\left(<30_{-0}^{+0}\right)$ & $<20_{-1}^{+1}$ & $<17_{-1}^{+1}$ & $<14_{-1}^{+1}$ & 1 \\
\hline ID31 & 7.4940 & $11.14_{-0.25}^{+0.22}$ & $74_{-15}^{+14}$ & $66_{-8}^{+6}$ & $53_{-3}^{+3}$ & $46_{-2}^{+2}$ & $\left(30_{-0}^{+0}\right)$ & $20_{-1}^{+1}$ & $17_{-1}^{+1}$ & $14_{-1}^{+1}$ & 4 \\
\hline $\mathrm{HZ} 2$ & 5.6697 & $<10.95_{-0.25}^{+0.22}$ & $<71_{-15}^{+13}$ & $<64_{-7}^{+6}$ & $<51_{-3}^{+3}$ & $<45_{-2}^{+2}$ & $\left(<29_{-0}^{+0}\right)$ & $<20_{-1}^{+1}$ & $<17_{-1}^{+1}$ & $<14_{-1}^{+1}$ & 1 \\
\hline
\end{tabular}

Notes.

a The $158 \mu \mathrm{m}$ fluxes used for predicting the fluxes at the other wavelengths are measured.

${ }^{\mathrm{b}}$ List of references: 1-Capak et al. (2015); 2-Barišić et al. (2017); 3-Willott et al. (2015); 4-Aravena et al. (2016).

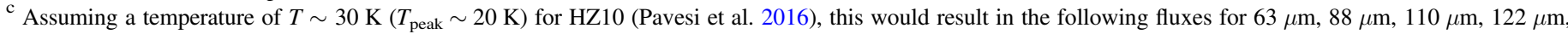
$190 \mu \mathrm{m}, 205 \mu \mathrm{m}$, and $220 \mu \mathrm{m}: 618 \mu \mathrm{Jy}, 1185 \mu \mathrm{Jy}, 1411 \mu \mathrm{Jy}, 1434 \mu \mathrm{Jy}, 1012 \mu \mathrm{Jy}, 902 \mu \mathrm{Jy}$, and $800 \mu \mathrm{Jy}$.

average temperature of such galaxies. Table 6 shows their expected (and measured, in the case of $158 \mu \mathrm{m}$ ) fluxes. We also include the other seven galaxies that are not detected in the FIR continuum. To predict the fluxes, we assumed an MIR powerlaw slope of $1.5<\alpha<2.5$, an emissivity of $1<\beta_{\mathrm{IR}}<2$, and a temperature $60 \mathrm{~K}<T<90 \mathrm{~K}\left(35 \mathrm{~K}<T_{\text {peak }}<50 \mathrm{~K}\right)$. For $25 \mathrm{~K}<T<45 \mathrm{~K}\left(18 \mathrm{~K}<T_{\text {peak }}<28 \mathrm{~K}\right)$, as assumed in $\mathrm{C} 15$, fluxes at wavelengths blueward of [C II] would be lower while fluxes redward of [C II] would be larger because of the shift of the SED to lower temperatures. In the following, we assume the same ALMA configuration as in Section A.2.

HZ10. The temperature of HZ10 at $z \sim 5.65$ has already been estimated to be $T \sim 30 \mathrm{~K}\left(T_{\text {peak }} \sim 20 \mathrm{~K}\right.$; Pavesi et al. 2016). However, the uncertainty of this estimate is large since only data at $>158 \mu \mathrm{m}$ are available. A better temperature estimate can be achieved by a $17 \sigma$ measurement in $122 \mu \mathrm{m}$. This would uniquely differentiate a temperature of $T=30 \mathrm{~K}$ and $T=60 \mathrm{~K} \quad(T=90 \mathrm{~K})$ with $2 \sigma \quad(3 \sigma)$ significance (Figure 16). With an estimated flux of $\sim 2000 \mu \mathrm{Jy}$ at $122 \mu \mathrm{m}$, a $17 \sigma$ measurement can be achieved in $\sim 20-30$ minutes of observing time in Band 7 (see Figures 18 and 15). Assuming a temperature prior of $25 \mathrm{~K}<T<45 \mathrm{~K}$ instead, we would expect a flux sensitivity of $\sim 1400 \mu \mathrm{Jy}$ and a required integration time of $\sim 40-50$ minutes.

HZ9. For the second brightest galaxy in FIR at $z \sim 5.55$, the rest-frame $122 \mu \mathrm{m}$ cannot be observed as it falls between Bands 7 and 8 . Similarly, 63 and $88 \mu \mathrm{m}$ are out of range. Instead, a $20 \sigma$ continuum measurement at $205 \mu \mathrm{m}$ can uniquely differentiate a temperature of $T=30 \mathrm{~K}$ and $T=60 \mathrm{~K} / 90 \mathrm{~K}$ with $1 \sigma$ significance. With an estimated flux of $\sim 300 \mu \mathrm{Jy}$ at $205 \mu \mathrm{m}$, a $20 \sigma$ measurement can be achieved in $\sim 1.5 \mathrm{hr}$ of observation in Band 6. Assuming a temperature prior of $25 \mathrm{~K}<T<45 \mathrm{~K}$ instead, we would expect a flux of $\sim 370 \mu \mathrm{Jy}$, which would decrease the integration time to $\sim 1 \mathrm{hr}$.

HZ4. HZ4 is at the same redshift as HZ9 and therefore cannot be observed at $122 \mu \mathrm{m}$. Also, $63 \mu \mathrm{m}$ and $88 \mu \mathrm{m}$ are out of the wavelength range. The $20 \sigma$ measurement at $205 \mu \mathrm{m}$ that is needed to uniquely differentiate a temperature of $T=30 \mathrm{~K}$ 
and $T=60 \mathrm{~K} / 90 \mathrm{~K}$ with $1 \sigma$ significance can only be achieved at integration times of more than $5 \mathrm{hr}$ for the estimated $205 \mu \mathrm{m}$ continuum flux of $\sim 110 \mu \mathrm{Jy}$. The same is true for the source WMH5 detected by Willott et al. (2015).

HZ6. HZ6 at $z \sim 5.3$ has been observed at $205 \mu \mathrm{m}$ by Pavesi et al. (2016) and a temperature of $T=60_{-27}^{+35} \mathrm{~K}\left(T_{\text {peak }} \sim 35 \mathrm{~K}\right)$ has been derived with a large uncertainty. Note that these measurements are integrated over all three components. HZ6 can be re-observed at $122 \mu \mathrm{m}$ (expected flux is $340 \mu \mathrm{Jy}$ ), reaching a $6 \sigma$ continuum detection in $2.5 \mathrm{hr}$. This results in a splitting of $T=30 \mathrm{~K}$ and $T=90 \mathrm{~K}$ at the $1 \sigma$ level. The $20 \sigma$ measurement at $205 \mu \mathrm{m}$ that is needed to uniquely differentiate a temperature of $T=30 \mathrm{~K}$ and $T=60 \mathrm{~K} / 90 \mathrm{~K}$ with $1 \sigma$ significance can only be achieved at integration times of more than $5 \mathrm{hr}$ for the estimated $205 \mu \mathrm{m}$ continuum flux of $\sim 126 \mu \mathrm{Jy}$ (c.f. $110 \pm 4 \mu \mathrm{Jy}$ as measured by Pavesi et al. 2016).

FIR-undetected galaxies. The $158 \mu \mathrm{m}$ continuum flux is not known for these galaxies, and therefore only a lower limit in integration time can be inferred. Taking at face value the continuum flux limit of the stacked observations $(\sim 35 \mu \mathrm{Jy})$, an integration time of at least $12 \mathrm{hr}$ is needed for a continuum detection of $5 \sigma$ at $158 \mu \mathrm{m}$ as shown in the large panel in Figure 18. Note that this figure was made for $z=5.9$, but due to the flat transmission function around rest-frame $158 \mu \mathrm{m}$ (Figure 15), the integration time estimates also hold for $z \sim 5.5$. For a $5 \sigma$ detection at $158 \mu \mathrm{m}$ (instead of $10 \sigma$ ) in Figure 17, the resulting integration times would increase by a factor of 4 . Thus, for a $5 \sigma$ detection at $205 \mu \mathrm{m}$, Figure 17 suggests four to eight times the integration time in $158 \mu \mathrm{m}$, so more than $50 \mathrm{hr}$. Thus, estimating the temperature for these FIR-undetected sources is not feasible with today's capabilities. This is also true for the $158 \mu \mathrm{m}$ continuum-detected candidates found in ALMA blind searches (Aravena et al. 2016) as well as for CLM1 from Willott et al. (2015).

\section{ORCID iDs}

Andreas L. Faisst (iD https://orcid.org/0000-0002-9382-9832 Lin Yan (D) https://orcid.org/0000-0003-1710-9339 Riccardo Pavesi (i) https://orcid.org/0000-0002-2263-646X Dominik A. Riechers (iD https://orcid.org/0000-00019585-1462

Ivana Barišić (iD https://orcid.org/0000-0001-6371-6274

Kevin C. Cooke (i) https://orcid.org/0000-0002-2200-9845

Daniel C. Masters (iD https://orcid.org/0000-0001-5382-6138

\section{References}

Álvarez-Márquez, J., Burgarella, D., Heinis, S., et al. 2016, A\&A, 587, A122 Anders, P., \& Fritze-v. Alvensleben, U. 2003, A\&A, 401, 1063 Ando, M., Ohta, K., Iwata, I., et al. 2007, PASJ, 59, 717

Aravena, M., Decarli, R., Walter, F., et al. 2016, ApJ, 833, 71 Armus, L., Mazzarella, J. M., Evans, A. S., et al. 2009, PASP, 121, 559 Baker, A. J., Lutz, D., Genzel, R., Tacconi, L. J., \& Lehnert, M. D. 2001, A\&A, 372, L37

Baldwin, J. A., Phillips, M. M., \& Terlevich, R. 1981, PASP, 93, 5

Barišić, I., Faisst, A. L., Capak, P. L., et al. 2017, ApJ, 845, 41

Battisti, A. J., Calzetti, D., \& Chary, R.-R. 2017, ApJ, 840, 109

Bendo, G. J., Galliano, F., \& Madden, S. C. 2012, MNRAS, 423, 197

Béthermin, M., Daddi, E., Magdis, G., et al. 2015, A\&A, 573, A113

Bian, F., Kewley, L., Dopita, M., \& Blanc, G. 2017, ApJ, 834, 51

Boquien, M., Buat, V., Boselli, A., et al. 2012, A\&A, 539, A145

Boquien, M., Calzetti, D., Kennicutt, R., et al. 2009, ApJ, 706, 553

Bourne, N., Dunlop, J. S., Merlin, E., et al. 2017, MNRAS, 467, 1360

Bouwens, R. J., Aravena, M., Decarli, R., et al. 2016, ApJ, 833, 72

Bouwens, R. J., Illingworth, G. D., Franx, M., et al. 2009, ApJ, 705, 936
Bouwens, R. J., Illingworth, G. D., Oesch, P. A., et al. 2012, ApJ, 754, 83 Bruzual, G., \& Charlot, S. 2003, MNRAS, 344, 1000

Buat, V., Giovannoli, E., Burgarella, D., et al. 2010, MNRAS, 409, L1 Buat, V., Iglesias-Páramo, J., Seibert, M., et al. 2005, ApJL, 619, L51 Buat, V., Noll, S., Burgarella, D., et al. 2012, A\&A, 545, A141 Burgarella, D., Buat, V., \& Iglesias-Páramo, J. 2005, MNRAS, 360, 1413 Calzetti, D., Armus, L., Bohlin, R. C., et al. 2000, ApJ, 533, 682 Capak, P. L., Carilli, C., Jones, G., et al. 2015, Natur, 522, 455 Capak, P. L., Riechers, D., Scoville, N. Z., et al. 2011, Natur, 470, 233 Cardamone, C., Schawinski, K., Sarzi, M., et al. 2009, MNRAS, 399, 1191 Carilli, C. L., Chluba, J., Decarli, R., et al. 2016, ApJ, 833, 73

Carilli, C. L., \& Walter, F. 2013, ARA\&A, 51, 105

Casey, C. M. 2012, MNRAS, 425, 3094

Casey, C. M., Scoville, N. Z., Sanders, D. B., et al. 2014, ApJ, 796, 95 Chabrier, G. 2003, PASP, 115, 763

Chapman, S. C., Helou, G., Lewis, G. F., \& Dale, D. A. 2003, ApJ, 588, 186 Charlot, S., \& Fall, S. M. 2000, ApJ, 539, 718

Chu, J. K., Sanders, D. B., Larson, K. L., et al. 2017, ApJS, 229, 25 Conley, A., Cooray, A., Vieira, J. D., et al. 2011, ApJL, 732, L35 Coppin, K., Pope, A., Menéndez-Delmestre, K., et al. 2010, ApJ, 713, 503 Coppin, K. E. K., Geach, J. E., Almaini, O., et al. 2015, MNRAS, 446, 1293 Cormier, D., Madden, S. C., Lebouteiller, V., et al. 2015, A\&A, 578, A53

Cortese, L., Boselli, A., Buat, V., et al. 2006, ApJ, 637, 242

Cowie, L. L., Barger, A. J., \& Hu, E. M. 2010, ApJ, 711, 928

Cowie, L. L., Barger, A. J., \& Hu, E. M. 2011, ApJ, 738, 136

Curti, M., Cresci, G., Mannucci, F., et al. 2017, MNRAS, 465, 1384 da Cunha, E., Groves, B., Walter, F., et al. 2013, ApJ, 766, 13

Daddi, E., Dickinson, M., Morrison, G., et al. 2007, ApJ, 670, 156 Dale, D. A., Aniano, G., Engelbracht, C. W., et al. 2012, ApJ, 745, 95 De Barros, S., Reddy, N., \& Shivaei, I. 2016, ApJ, 820, 96 de Barros, S., Schaerer, D., \& Stark, D. P. 2014, A\&A, 563, A81 De Looze, I., Cormier, D., Lebouteiller, V., et al. 2014, A\&A, 568, A62 Díaz-Santos, T., Armus, L., Charmandaris, V., et al. 2014, ApJL, 788, L17 Draine, B. T. 2006, ApJ, 636, 1114

Dunlop, J. S. 2016, Msngr, 166, 48

Dunne, L., Eales, S., Edmunds, M., et al. 2000, MNRAS, 315, 115 Elbaz, D., Daddi, E., Le Borgne, D., et al. 2007, A\&A, 468, 33 Elmegreen, D. M., Elmegreen, B. G., Marcus, M. T., et al. 2009, ApJ, 701, 306 Erb, D. K., Pettini, M., Steidel, C. C., et al. 2016, ApJ, 830, 52 Faber, S. M., Phillips, A. C., Kibrick, R. I., et al. 2003, Proc. SPIE, 4841, 1657 Faisst, A. L. 2016, ApJ, 829, 99

Faisst, A. L., Capak, P., Hsieh, B. C., et al. 2016a, ApJ, 821, 122 Faisst, A. L., Capak, P. L., Davidzon, I., et al. 2016b, ApJ, 822, 29 Feldmann, R. 2015, MNRAS, 449, 3274

Fitzpatrick, E. L. 1986, AJ, 92, 1068

Förster Schreiber, N. M., Shapley, A. E., Genzel, R., et al. 2011, ApJ, 739, 45 Fudamoto, et al. 2017, MNRAS, submitted (arXiv:1705.01559)

Genzel, R., Tacconi, L. J., Lutz, D., et al. 2015, ApJ, 800, 20 Gil de Paz, A., Boissier, S., Madore, B. F., et al. 2007, ApJS, 173, 185 González, V., Bouwens, R., Illingworth, G., et al. 2014, ApJ, 781, 34 González-López, J., Riechers, D. A., Decarli, R., et al. 2014, ApJ, 784, 99 Gordon, K. D., Witt, A. N., Rudy, R. J., et al. 2000, ApJ, 544, 859 Green, J. C., Froning, C. S., Osterman, S., et al. 2012, ApJ, 744, 60 Greis, S. M. L., Stanway, E. R., Davies, L. J. M., \& Levan, A. J. 2016, MNRAS, 459, 2591

Greve, T. R., Vieira, J. D., Weiß, A., et al. 2012, ApJ, 756, 101 Heinis, S., Buat, V., Béthermin, M., et al. 2013, MNRAS, 429, 1113 Heinis, S., Buat, V., Béthermin, M., et al. 2014, MNRAS, 437, 1268 Hemmati, S., Miller, S. H., Mobasher, B., et al. 2014, ApJ, 797, 108 Herrera-Camus, R., Bolatto, A. D., Wolfire, M. G., et al. 2015, ApJ, 800, 1 Hodge, J. A., Riechers, D., Decarli, R., et al. 2015, ApJL, 798, L18 Hodge, J. A., Swinbank, A. M., Simpson, J. M., et al. 2016, ApJ, 833, 103 Hopkins, P. F., Younger, J. D., Hayward, C. C., Narayanan, D., \& Hernquist, L. 2010, MNRAS, 402, 1693

Howell, J. H., Armus, L., Mazzarella, J. M., et al. 2010, ApJ, 715, 572 Hu, E. M., Cowie, L. L., Kakazu, Y., \& Barger, A. J. 2009, ApJ, 698, 2014 Ilbert, O., Arnouts, S., Le Floc'h, E., et al. 2015, A\&A, 579, A2 Jiang, L., Finlator, K., Cohen, S. H., et al. 2016, ApJ, 816, 16 Kanekar, N., Wagg, J., Ram Chary, R., \& Carilli, C. L. 2013, ApJL, 771, L20 Karim, A., Schinnerer, E., Martínez-Sansigre, A., et al. 2011, ApJ, 730, 61 Kennicutt, R. C., Calzetti, D., Aniano, G., et al. 2011, PASP, 123, 1347 Kewley, L. J., \& Ellison, S. L. 2008, ApJ, 681, 1183

Kewley, L. J., Maier, C., Yabe, K., et al. 2013, ApJL, 774, L10 Knudsen, K. K., Richard, J., Kneib, J.-P., et al. 2016, MNRAS, 462, L6 Knudsen, K. K., Watson, D., Frayer, D., et al. 2017, MNRAS, 466, 138 Kong, X., Charlot, S., Brinchmann, J., \& Fall, S. M. 2004, MNRAS, 349, 769 
Laigle, C., McCracken, H. J., Ilbert, O., et al. 2016, ApJS, 224, 24 Larson, K. L., Sanders, D. B., Barnes, J. E., et al. 2016, ApJ, 825, 128 Lee, K.-S., Alberts, S., Atlee, D., et al. 2012, ApJL, 758, L31 Lee, N., Sanders, D. B., Casey, C. M., et al. 2015, ApJ, 801, 80 Lilly, S. J., Le Fèvre, O., Renzini, A., et al. 2007, ApJS, 172, 70 Lofthouse, E. K., Houghton, R. C. W., \& Kaviraj, S. 2017, MNRAS, 471, 2311 Ma, J., Gonzalez, A. H., Vieira, J. D., et al. 2016, ApJ, 832, 114 Madden, S. C., Rémy-Ruyer, A., Galametz, M., et al. 2013, PASP, 125, 600 Magdis, G. E., Daddi, E., Béthermin, M., et al. 2012, ApJ, 760, 6 Magnelli, B., Lutz, D., Saintonge, A., et al. 2014, A\&A, 561, A86 Maiolino, R., Carniani, S., Fontana, A., et al. 2015, MNRAS, 452, 54 Maiolino, R., Nagao, T., Grazian, A., et al. 2008, A\&A, 488, 463

Mannucci, F., Cresci, G., Maiolino, R., Marconi, A., \& Gnerucci, A. 2010, MNRAS, 408, 2115

Mármol-Queraltó, E., McLure, R. J., Cullen, F., et al. 2016, MNRAS, 460, 3587

Martin, D. C., Fanson, J., Schiminovich, D., et al. 2005, ApJL, 619, L1

Mason, C. A., Treu, T., Fontana, A., et al. 2017, ApJ, 838, 14

Masters, D., Faisst, A., \& Capak, P. 2016, ApJ, 828, 18

Meurer, G. R., Heckman, T. M., \& Calzetti, D. 1999, ApJ, 521, 64

Meurer, G. R., Heckman, T. M., Leitherer, C., et al. 1995, AJ, 110, 2665

Miller, T. B., Chapman, S. C., Hayward, C. C., et al. 2016, arXiv:1611.08552

Muñoz-Mateos, J. C., Gil de Paz, A., Boissier, S., et al. 2009, ApJ, 701 1965

Narayanan, D., Dave, R., Johnson, B., et al. 2017, arXiv:1705.05858

Noeske, K. G., Weiner, B. J., Faber, S. M., et al. 2007, ApJL, 660, L43

Oke, J. B. 1974, ApJS, 27, 21

Ota, K., Walter, F., Ohta, K., et al. 2014, ApJ, 792, 34

Ouchi, M., Ellis, R., Ono, Y., et al. 2013, ApJ, 778, 102

Overzier, R. A., Heckman, T. M., Wang, J., et al. 2011, ApJL, 726, L7

Pannella, M., Carilli, C. L., Daddi, E., et al. 2009, ApJL, 698, L116

Pannella, M., Elbaz, D., Daddi, E., et al. 2015, ApJ, 807, 141

Pavesi, R., Riechers, D. A., Capak, P. L., et al. 2016, ApJ, 832, 151

Peng, C. Y., Ho, L. C., Impey, C. D., \& Rix, H.-W. 2011, GALFIT: Detailed Structural Decomposition of Galaxy Images, Astrophysics Source Code Library, ascl: 1104.010

Pettini, M., Kellogg, M., Steidel, C. C., et al. 1998, ApJ, 508, 539

Pettini, M., \& Pagel, B. E. J. 2004, MNRAS, 348, L59

Pilyugin, L. S., \& Thuan, T. X. 2005, ApJ, 631, 231

Pineda, J. L., Langer, W. D., Velusamy, T., \& Goldsmith, P. F. 2013, A\&A, 554, A103

Pope, A., Chary, R.-R., Alexander, D. M., et al. 2008, ApJ, 675, 1171

Popping, G., Puglisi, A., \& Norman, C. A. 2017b, MNRAS (arXiv:1706. 06587)

Popping, G., Somerville, R. S., \& Galametz, M. 2017a, MNRAS, 471, 3152

Prestwich, A. H., Tsantaki, M., Zezas, A., et al. 2013, ApJ, 769, 92

Prevot, M. L., Lequeux, J., Prevot, L., Maurice, E., \& Rocca-Volmerange, B. 1984, A\&A, 132, 389

Rasappu, N., Smit, R., Labbé, I., et al. 2016, MNRAS, 461, 3886

Reddy, N., Dickinson, M., Elbaz, D., et al. 2012, ApJ, 744, 154

Reddy, N. A., Erb, D. K., Pettini, M., Steidel, C. C., \& Shapley, A. E. 2010 , ApJ, 712, 1070

Reddy, N. A., Kriek, M., Shapley, A. E., et al. 2015, ApJ, 806, 259

Reddy, N. A., Steidel, C. C., Fadda, D., et al. 2006, ApJ, 644, 792

Rémy-Ruyer, A., Madden, S. C., Galliano, F., et al. 2013, A\&A, 557, A95
Rich, J. A., Torrey, P., Kewley, L. J., Dopita, M. A., \& Rupke, D. S. N. 2012, ApJ, 753, 5

Riechers, D. A., Bradford, C. M., Clements, D. L., et al. 2013, Natur, 496, 329

Riechers, D. A., Carilli, C. L., Capak, P. L., et al. 2014, ApJ, 786, 31

Rupke, D. S. N., Veilleux, S., \& Baker, A. J. 2008, ApJ, 674, 172

Safarzadeh, M., Hayward, C. C., \& Ferguson, H. C. 2017, ApJ, 840, 15

Sanders, D. B., Mazzarella, J. M., Kim, D.-C., Surace, J. A., \& Soifer, B. T. 2003, AJ, 126, 1607

Sanders, R. L., Shapley, A. E., Kriek, M., et al. 2016, ApJL, 825, L23

Schaerer, D., Boone, F., Zamojski, M., et al. 2015, A\&A, 574, A19

Schaerer, D., de Barros, S., \& Sklias, P. 2013, A\&A, 549, A4

Schneider, R., Hunt, L., \& Valiante, R. 2016, MNRAS, 457, 1842

Schreiber, C., Pannella, M., Elbaz, D., et al. 2015, A\&A, 575, A74

Schreiber, C., Pannella, M., Leiton, R., et al. 2017, A\&A, 599, A134

Scoville, N., Aussel, H., Brusa, M., et al. 2007, ApJS, 172, 1

Scoville, N., Faisst, A., Capak, P., et al. 2015, ApJ, 800, 108

Scoville, N., Sheth, K., Aussel, H., et al. 2016, ApJ, 820, 83

Scoville, N. Z. 2013, in Secular Evolution of Galaxies, ed. J. Falcón-Barroso \&

J. H. Knapen (Cambridge: Cambridge Univ. Press), 491

Scoville, N. Z., \& Kwan, J. 1976, ApJ, 206, 718

Seaton, M. J. 1979, MNRAS, 187, 73P

Seibert, M., Martin, D. C., Heckman, T. M., et al. 2005, ApJL, 619, L55

Shim, H., Chary, R.-R., Dickinson, M., et al. 2011, ApJ, 738, 69

Shivaei, I., Reddy, N. A., Shapley, A. E., et al. 2015, ApJ, 815, 98

Siana, B., Smail, I., Swinbank, A. M., et al. 2009, ApJ, 698, 1273

Skibba, R. A., Engelbracht, C. W., Dale, D., et al. 2011, ApJ, 738, 89

Sklias, P., Zamojski, M., Schaerer, D., et al. 2014, A\&A, 561, A149

Speagle, J. S., Steinhardt, C. L., Capak, P. L., \& Silverman, J. D. 2014, ApJS, 214, 15

Stanway, E. R., Eldridge, J. J., Greis, S. M. L., et al. 2014, MNRAS, 444, 3466

Stark, D. P., Schenker, M. A., Ellis, R., et al. 2013, ApJ, 763, 129

Steidel, C. C., Rudie, G. C., Strom, A. L., et al. 2014, ApJ, 795, 165

Steinhardt, C. L., Speagle, J. S., Capak, P., et al. 2014, ApJL, 791, L25

Strandet, M. L., Weiss, A., Vieira, J. D., et al. 2016, ApJ, 822, 80

Strom, A. L., Steidel, C. C., Rudie, G. C., et al. 2017, ApJ, 836, 164

Tacchella, S., Lang, P., Carollo, C. M., et al. 2015, ApJ, 802, 101

Tacconi, L. J., Genzel, R., Neri, R., et al. 2010, Natur, 463, 781

Takeuchi, T. T., Buat, V., Heinis, S., et al. 2010, A\&A, 514, A4

Takeuchi, T. T., Yuan, F.-T., Ikeyama, A., Murata, K. L., \& Inoue, A. K. 2012, ApJ, 755, 144

Tasca, L. A. M., Le Fèvre, O., Hathi, N. P., et al. 2015, A\&A, 581, A54

Temi, P., Brighenti, F., \& Mathews, W. G. 2009, ApJ, 707, 890

To, C.-H., Wang, W.-H., Owen, F. N., et al. 2014, ApJ, 792, 139

U, V., Sanders, D. B., Mazzarella, J. M., et al. 2012, ApJS, 203, 9

Vallini, L., Ferrara, A., Pallottini, A., \& Gallerani, S. 2017, MNRAS, 467, 1300

Vallini, L., Gallerani, S., Ferrara, A., \& Baek, S. 2013, MNRAS, 433, 1567

Vallini, L., Gallerani, S., Ferrara, A., Pallottini, A., \& Yue, B. 2015, ApJ, 813,36

Walter, F., Decarli, R., Carilli, C., et al. 2012, ApJ, 752, 93

Watson, D., Christensen, L., Knudsen, K. K., et al. 2015, Natur, 519, 327

Weiß, A., De Breuck, C., Marrone, D. P., et al. 2013, ApJ, 767, 88

Willott, C. J., Carilli, C. L., Wagg, J., \& Wang, R. 2015, ApJ, 807, 180 